MÉMOIRES DE LA SMF 87

ALBANESE AND PICARD 1-MOTIVES

Luca Barbieri-Viale

Vasudevan Srinivas

Société Mathématique de France 2001

Publié avec le concours du Centre National de la Recherche Scientifique 


\section{Barbieri-Viale}

Dipartimento di Metodi e Modelli Matematici, Università degli Studi di Roma "La Sapienza", Via A. Scarpa, 16, I-00161 Roma, Italia.

E-mail : barbieri@dmmm. uniroma1.it

Url : http: //www.dmmm. uniroma1.it/

V. Srinivas

School of Mathematics, Tata Institute of Fundamental Research, Homi Bhabha Road, Mumbai-400005, India.

E-mail : srinivas@math.tifr.res.in

Url : http: //www.math.tifr.res.in/

2000 Mathematics Subject Classification. - 14F42, 14C30, 32S35, $19 \mathrm{E} 15$.

Key words and phrases. - Hodge theory, motives, algebraic cycles, singularities.

This work has been done during periods of stay at several research institutions including University of Paris VII and Institut Henri Poincaré in Paris, T.I.F.R. in Mumbai, DI.MA in Genoa and I.C.T.P. in Trieste which we thank for the hospitality.

The second author wishes to acknowledge support from the Swarnajayanthi Fellowship of the D.S.T. and the IFCPAR Project "Geometry"(No. 1601-2), which made possible a visit to the University of Paris VII, concurrently with a visit of the first author to the Institut Henri Poincaré, and enabled useful discussions on this work with O. Gabber, L. Illusie, F. Oort and M. Raynaud. In particular, we thank Raynaud for providing us with the reference $[\mathbf{5 3}]$.

The first author is deeply grateful to the Italian C.N.R. and M.U.R.S.T., the European Union Science Plan "K-Theory and Algebraic Groups" and the Tata Institute of Fundamental Research for financial support. 


\title{
ALBANESE AND PICARD 1-MOTIVES
}

\author{
Luca Barbieri-Viale, Vasudevan Srinivas
}

\begin{abstract}
Let $X$ be an $n$-dimensional algebraic variety over a field of characteristic zero. We describe algebraically defined Deligne 1-motives $\mathrm{Alb}^{+}(X), \mathrm{Alb}^{-}(X)$, $\mathrm{Pic}^{+}(X)$ and $\mathrm{Pic}^{-}(X)$ which generalize the classical Albanese and Picard varieties of a smooth projective variety. We compute Hodge, $\ell$-adic and De Rham realizations proving Deligne's conjecture for $H^{2 n-1}, H_{2 n-1}, H^{1}$ and $H_{1}$.

We investigate functoriality, universality, homotopical invariance and invariance under formation of projective bundles. We compare our cohomological and homological 1-motives for normal schemes. For proper schemes, we obtain an Abel-Jacobi map from the (Levine-Weibel) Chow group of zero cycles to our cohomological Albanese 1-motive which is the universal regular homomorphism to semi-abelian varieties. By using this universal property we get "motivic" Gysin maps for projective local complete intersection morphisms.
\end{abstract}

Résumé (1-motifs d'Albanese et de Picard). - Soit $X$ une variété algébrique de dimension $n$ sur un corps de caractéristique 0. Nous décrivons les 1-motifs de Deligne $\mathrm{Alb}^{+}(X), \mathrm{Alb}^{-}(X), \mathrm{Pic}^{+}(X)$ et $\mathrm{Pic}^{-}(X)$ définis algébriquement, qui généralisent les variétés d'Albanese et de Picard classiques d'une variété projective lisse. Nous calculons les réalisations de Hodge, $\ell$-adique et de De Rham, montrant ainsi la conjecture de Deligne pour $H^{2 n-1}, H_{2 n-1}, H^{1}$ et $H_{1}$.

Nous étudions la fonctorialité, l'universalité, l'invariance par homotopie et l'invariance par formation de fibrés projectifs. Nous comparons nos 1-motifs homologiques et cohomologiques pour les schémas normaux. Pour des schémas propres, nous obtenons une application d'Abel-Jacobi du groupe de (Levine-Weibel) Chow des zérocycles vers notre 1-motif cohomologique d'Albanese, qui est l'homomorphisme universel régulier vers les variétés semi-abéliennes. En utilisant cette propriété universelle, nous obtenons des applications de Gysin «motiviques » pour les morphismes projectifs localement intersection complète. 



\section{CONTENTS}

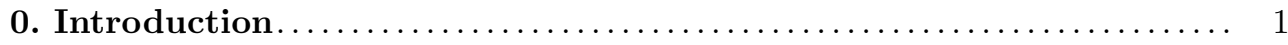

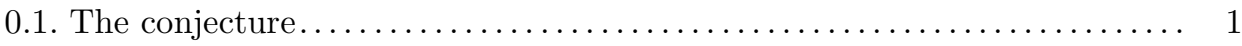

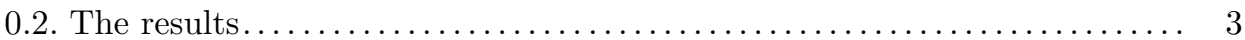

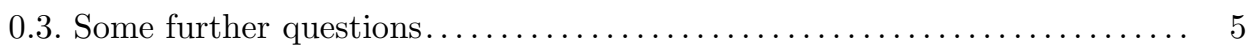

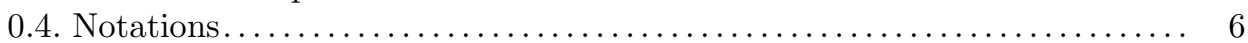

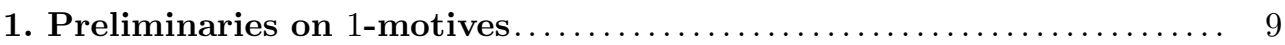

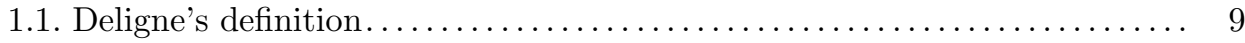

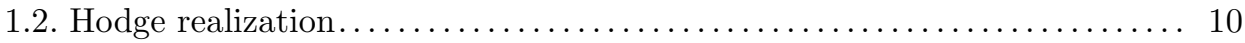

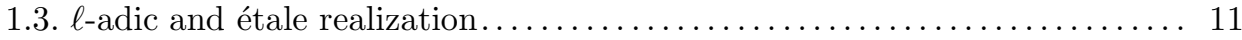

1.4. De Rham realization................................... 13

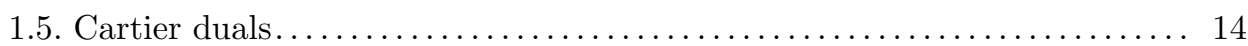

2. Homological Picard 1-motive: $\mathrm{Pic}^{-} \ldots \ldots \ldots \ldots \ldots \ldots \ldots \ldots \ldots \ldots$

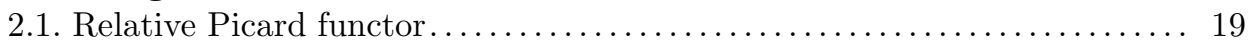

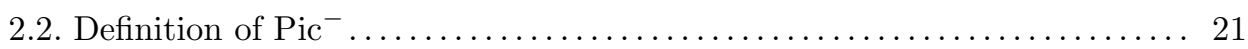

2.3. Independence of resolutions and compactifications................ 22

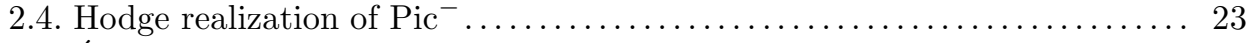

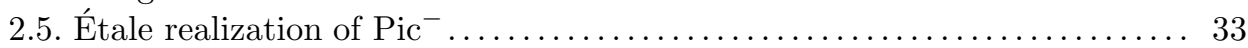

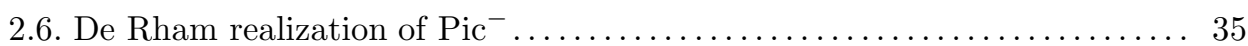

3. Cohomological Albanese 1-motive: $\mathrm{Alb}^{+} \ldots \ldots \ldots \ldots \ldots \ldots \ldots \ldots$

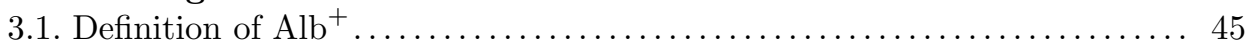

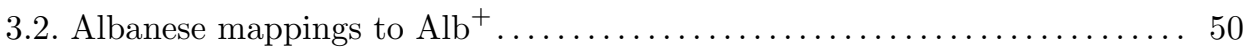

3.3. Hodge, étale and De Rham realizations of $\mathrm{Alb}^{+} \ldots \ldots \ldots \ldots \ldots \ldots \ldots \ldots$

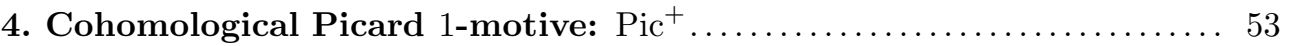

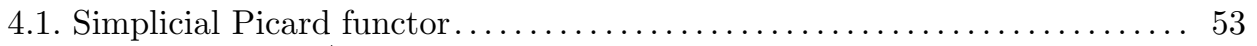

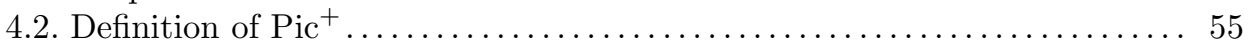

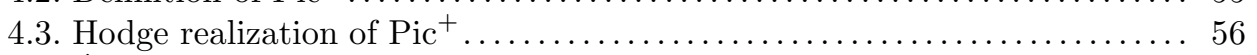

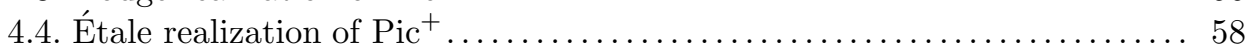

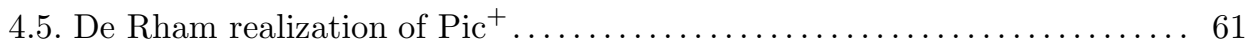


5. Homological Albanese 1-motive: $\mathrm{Alb}^{-} \ldots \ldots \ldots \ldots \ldots \ldots \ldots \ldots \ldots \ldots$

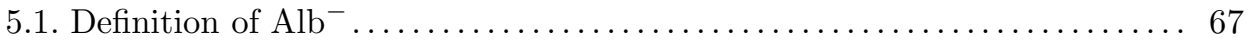

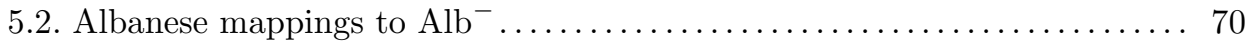

5.3. Hodge, étale and De Rham realizations of $\mathrm{Alb}^{-} \ldots \ldots \ldots \ldots \ldots \ldots \ldots \ldots$

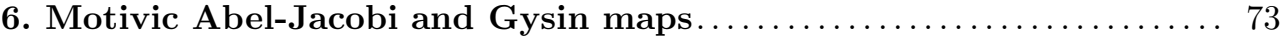

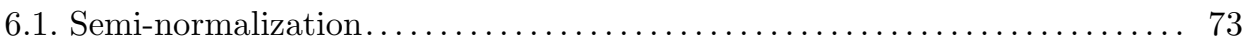

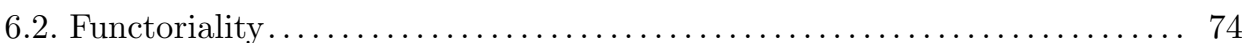

6.3. Projective bundles and vector bundles.................... 79

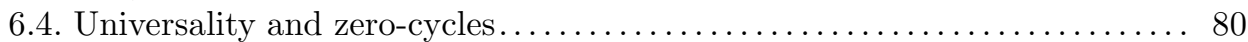

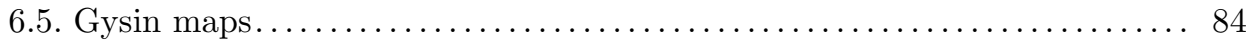

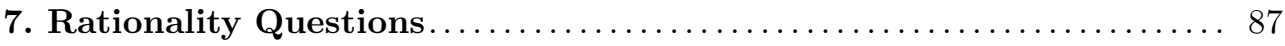

7.1. 1-motives over non-closed fields........................ 87

7.2. Albanese and Picard 1-motives in zero characteristic ............. 88

7.3. Albanese mappings.................................. 90

Appendix. Picard functors................................ 91

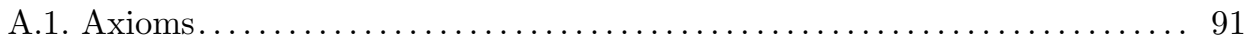

A.2. Representability of the relative Picard functor ................ 95

A.3. Representability of the simplicial Picard functor................... 96

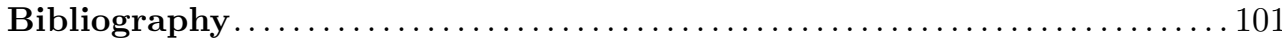




\section{CHAPTER 0}

\section{INTRODUCTION}

This work is motivated by Deligne's conjecture that 1-motives obtained from the mixed Hodge structure on the cohomology of an algebraic variety would be "algebraically defined" (see $[\mathbf{1 5}, \S 10.4 .1]$ and $[\mathbf{1 6}])$. Deligne $([\mathbf{1 5}, \S 10.1 .3])$ observed that a torsion free mixed Hodge structure $H$ (i.e., such that $H_{\mathbb{Z}}$ is torsion-free), which is of Hodge type $\{(0,0),(0,-1),(-1,0),(-1,-1)\}$, and such that $\operatorname{gr}_{-1}^{W}(H)$ is polarizable, yields $i)$ a semi-abelian variety $G$, whose abelian quotient is the abelian variety given by $\operatorname{gr}_{-1}^{W}(H)$, together with $\left.i i\right)$ a homomorphism $u$ from the lattice $L=\operatorname{gr}_{0}^{W}\left(H_{\mathbb{Z}}\right)$ to the group $G(\mathbb{C})$, induced by the canonical map $H_{\mathbb{Z}} \rightarrow H_{\mathbb{C}}$.

Deligne called such a complex of group schemes $[L \stackrel{u}{\rightarrow} G]$ a 1-motive over $\mathbb{C}$, and showed that the category of 1 -motives over $\mathbb{C}$ is equivalent to the category of torsion free mixed Hodge structures of the above type. Thus any such 1-motive $M=[L \stackrel{u}{\rightarrow} G]$ has a Hodge realization $T_{\text {Hodge }}(M)$, i.e., there is a unique (up to isomorphism) torsionfree mixed Hodge structure $T_{\text {Hodge }}(M)$ such that $M$ can be obtained from $T_{\text {Hodge }}(M)$ as above. Deligne $([\mathbf{1 5}, \S 10.1 .11])$ also defined the $\ell$-adic and De Rham realizations of a 1-motive $M$, denoted by $T_{\ell}(M)$ and $T_{\mathrm{DR}}(M)$, respectively (see Chapter 1 for more details).

\subsection{The conjecture}

Deligne's conjecture, in particular, is that if $X$ is an $n$-dimensional algebraic variety over a field $k$ of characteristic 0 , then there are "algebraically defined" 1-motives, also defined over $k$, compatible with base change to extension fields, such that $i$ ) when $k=\mathbb{C}$, their Hodge realizations are respectively isomorphic to the mixed Hodge structures on

$H^{2 n-1}(X, \mathbb{Z}(n)) /($ torsion $), \quad H_{1}(X, \mathbb{Z}) /($ torsion $), \quad H^{1}(X, \mathbb{Z}(1)), \quad H_{2 n-1}(X, \mathbb{Z}(1-n))$

ii) if $k$ is algebraically closed, their $\ell$-adic and De Rham realizations are naturally isomorphic to the corresponding $\ell$-adic and De Rham (co)homology iii) the above 
identifications are compatible with other structures, like comparison isomorphisms, filtrations, Galois action, etc. Our goal is to prove these statements. Moreover, we obtain some geometric properties of our constructions.

We recall that the case $n=1$, i.e., when $X$ is a curve, is already treated by Deligne $([\mathbf{1 5}, \S 10.3])$, and the case when $X$ smooth and proper corresponds to the well known transcendental descriptions of the Albanese and Picard varieties. Our construction of Albanese and Picard 1-motives generalizes these cases. In the general case, for $n \geqslant 1$, we propose the following dictionary:

$\begin{array}{ll}\text { Mixed Hodge Structure } & \text { 1-Motive } \\ H^{2 n-1}(X, \mathbb{Z}(n)) & \operatorname{Alb}^{+}(X) \\ H_{2 n-1}(X, \mathbb{Z}(1-n)) & \operatorname{Pic}^{-}(X) \\ H^{1}(X, \mathbb{Z}(1)) & \operatorname{Pic}^{+}(X) \\ H_{1}(X, \mathbb{Z}) & \operatorname{Alb}^{-}(X)\end{array}$

Here, $\operatorname{Alb}^{+}(X)$ is the "cohomological Albanese" 1-motive obtained from the mixed Hodge structure $H^{2 n-1}(X, \mathbb{Z}(n)) /\left(\right.$ torsion) and, dually, $\operatorname{Pic}^{-}(X)$ is the "homological Picard" 1-motive obtained from $H_{2 n-1}(X, \mathbb{Z}(1-n)) /$ (torsion), etc. The 1-motive $\mathrm{Alb}^{+}(X)$ is the Cartier dual of $\mathrm{Pic}^{-}(X)$ and $\mathrm{Pic}^{+}(X)$ is the Cartier dual of $\mathrm{Alb}^{-}(X)$. In case $X$ is singular, we have that $\mathrm{Alb}^{+}(X) \neq \mathrm{Alb}^{-}(X)$ in general, because of the possible failure of Poincaré duality. When $n=1, \operatorname{Alb}^{+}(X)$ and $\operatorname{Pic}^{+}(X)$ (and dually, $\operatorname{Alb}^{-}(X)$ and $\left.\operatorname{Pic}^{-}(X)\right)$ coincide.

We recall that the geometric definition of the "cohomological Picard and homological Albanese" 1-motives of a smooth, but possibly non-proper scheme $X$, goes back to Serre's explicit construction of its Albanese variety, see [48]; in fact, Serre's Albanese variety was defined as the Cartier dual of the 1-motive

$$
\operatorname{Pic}^{+}(X) \stackrel{\text { def }}{=}\left[\operatorname{Div}_{Y}^{0}(\bar{X}) \rightarrow \operatorname{Pic}^{0}(\bar{X})\right] \quad(X \text { smooth })
$$

where $\bar{X}$ is a smooth compactification of $X$ with boundary $Y, \operatorname{Div}_{Y}^{0}(\bar{X})$ is the free abelian group of divisors which are algebraically equivalent to zero and supported on $Y$, being mapped canonically to $\operatorname{Pic}^{0}(\bar{X})$. On the other hand, a geometric construction of $\mathrm{Alb}^{+}$or $\mathrm{Pic}^{-}$for a smooth open variety is more difficult and it appears to be new as well.

Following the construction in [31], in the paper of Ramachandran [39] a geometric construction of $\mathrm{Pic}^{+}(X)$ and $\mathrm{Alb}^{-}(X)$ was proposed for varieties with a singular closed point obtained by collapsing a finite set of closed points in a smooth open variety; in a subsequent paper, see [40], he proposed, independently, definitions of Albanese and Picard motives corresponding to our $\mathrm{Pic}^{+}(X)$ and $\mathrm{Alb}^{-}(X)$. Ramachandran announced in [41] (cf. [3]) a proof of the algebraicity (up to isogeny) of certain 1motives built out of $H^{i}(X, \mathbb{Q}(1))$ for $i \leqslant \operatorname{dim} X+1$. 
Apart from Ramachandran's work, a related paper by Carlson [12] on analogues of Abel's theorem for $H^{2}$ of some singular surfaces (see also [22]) and the recent paper [3] (see also the "Hodge 1-motives" considered in [1] and related papers $[\mathbf{7}]$ and $[\mathbf{9}]$ ) we do not know any results on Deligne's conjecture (1972).

\subsection{The results}

Our definition of $\mathrm{Pic}^{-}(X)$ is roughly the following (see Sections 2.1 and 2.2 below for a more precise statement). Let $X$ be any equidimensional algebraic variety over an algebraically closed field $k$ of characteristic zero. Let $f: \widetilde{X} \rightarrow X$ be a resolution of singularities and let $\bar{X}$ be a smooth compactification of $\widetilde{X}$ with normal crossing boundary divisor $Y$.

Let $S$ be the singular locus of $X$ and let $\bar{S}$ be the closure of $f^{-1}(S)$ in $\bar{X}$. Then we let $\operatorname{Div} \frac{0}{S}(\bar{X}, Y)$ be the group of divisors supported on $\bar{S}$ which are $i$ ) disjoint from $Y$ (i.e., are linear combinations of compact components of $f^{-1}(S)$ ), and ii) are algebraically equivalent to zero relative to $Y$. We let $\operatorname{Div} \frac{0}{S / S}(\bar{X}, Y)$ be the subgroup of those divisors which have vanishing push-forward (as Weil divisors) along $f$.

We can show the existence of a group scheme $\operatorname{Pic}(\bar{X}, Y)$ associated to isomorphism classes of pairs $(\mathcal{L}, \varphi)$ such that $\mathcal{L}$ is a line bundle on $\bar{X}$ and $\varphi:\left.\mathcal{L}\right|_{Y} \cong \mathcal{O}_{Y}$ is a trivialization on $Y$. The connected component of the identity $\operatorname{Pic}^{0}(\bar{X}, Y)$ is a semiabelian variety, which can be represented as an extension

$$
0 \rightarrow \frac{H^{0}\left(Y, \mathcal{O}_{Y}^{*}\right)}{\operatorname{im} H^{0}\left(\bar{X}, \mathcal{O}_{\bar{X}}^{*}\right)} \rightarrow \operatorname{Pic}^{0}(\bar{X}, Y) \rightarrow \operatorname{ker}^{0}\left(\operatorname{Pic}^{0}(\bar{X}) \rightarrow \oplus_{i} \operatorname{Pic}^{0}\left(Y_{i}\right)\right) \rightarrow 0
$$

where $Y=\cup_{i} Y_{i}$ is expressed as a union of (smooth) irreducible components. The mapping which takes a divisor $D$ disjoint from $Y$ to the class of the pair $\left(\mathcal{O}_{\bar{X}}(D), 1\right)$ in $\operatorname{Pic}(\bar{X}, Y)$ yields the "homological Picard" 1-motive

$$
\operatorname{Pic}^{-}(X) \stackrel{\text { def }}{=}\left[\operatorname{Div} \frac{0}{S / S}(\bar{X}, Y) \rightarrow \operatorname{Pic}^{0}(\bar{X}, Y)\right] .
$$

The "cohomological Albanese" 1-motive $\mathrm{Alb}^{+}(X)$ is defined to be the Cartier dual of $\mathrm{Pic}^{-}(X)$ (see Section 3.1); a "concrete" description of it is also given when $X$ is either smooth or proper.

The definition of $\mathrm{Pic}^{+}(X)$ is obtained by generalizing Serre's construction of the generalized Albanese variety to smooth simplicial schemes (see Sections 4.1 and 4.2 for the details). Let $X$ be a variety over an algebraically closed field $k$ of characteristic 0 . Let $X$. be a smooth proper hypercovering of $X$, and consider a proper smooth compactification $\bar{X}$. with normal crossing boundary $Y$. (we refer to $[\mathbf{1 5}, \S 6.2]$ for the existence of such a hypercovering and compactification). Let $\operatorname{Div}_{Y}(\bar{X} \cdot)$ be the subgroup of divisors on $\bar{X}_{0}$ supported on $Y_{0}$ with zero pull-back on $\bar{X}_{1}$, i.e., by definition

$$
\operatorname{Div}_{Y_{\bullet}}(\bar{X} \cdot) \stackrel{\text { def }}{=} \operatorname{ker}\left(\operatorname{Div}_{Y_{0}}\left(\bar{X}_{0}\right) \stackrel{d_{0}^{*}-d_{1}^{*}}{\longrightarrow} \operatorname{Div}_{Y_{1}}\left(\bar{X}_{1}\right)\right)
$$


We consider the simplicial Picard functor

$$
T \longmapsto \operatorname{Pic}(T \times \bar{X} \cdot) \stackrel{\text { def }}{=} \mathbb{H}^{1}\left(T \times \bar{X} \cdot, \mathcal{O}_{T \times \bar{X}}^{*}\right)
$$

and we show that the associated sheaf $\mathbf{P i c} \bar{X}_{\bar{X}} . / k$ (with respect to the fpqc-topology) is representable by a group scheme locally of finite type over $k$, whose connected component of the identity $\mathbf{P i c}_{\bar{X}}^{0}{ }_{\text {. } / k}$ over $k=\bar{k}$ is an extension of the abelian scheme $\operatorname{ker}^{0}\left(\operatorname{Pic}_{\bar{X}_{0} / k}^{0} \rightarrow \operatorname{Pic}_{\bar{X}_{1} / k}^{0}\right)$ by the torus given by

$$
\frac{\operatorname{ker}\left(H^{0}\left(\bar{X}_{1}, \mathcal{O}_{\bar{X}_{1}}^{*}\right) \rightarrow H^{0}\left(\bar{X}_{2}, \mathcal{O}_{\bar{X}_{2}}^{*}\right)\right)}{\operatorname{im}\left(H^{0}\left(\bar{X}_{0}, \mathcal{O}_{\bar{X}_{0}}^{*}\right) \rightarrow H^{0}\left(\bar{X}_{1}, \mathcal{O}_{\bar{X}_{1}}^{*}\right)\right)}
$$

Let $\operatorname{Div}_{Y \cdot}^{0}(\bar{X} \cdot)$ denote the subgroup of those divisors which are mapped to $\mathbf{P i c}_{\bar{X}}^{0}{ }_{\bullet}(k)$ under the canonical mapping. We then define the "cohomological Picard" 1-motive of the variety $X$ as

$$
\operatorname{Pic}^{+}(X) \stackrel{\text { def }}{=}\left[\operatorname{Div}_{Y .}^{0}(\bar{X} .) \rightarrow \operatorname{Pic}^{0}(\bar{X} \cdot)\right]
$$

The "homological Albanese" 1-motive $\mathrm{Alb}^{-}(X)$ is defined to be the Cartier dual of $\operatorname{Pic}^{+}(X)$ (see Section 5.1).

We show that $\mathrm{Pic}^{-}(X), \mathrm{Alb}^{+}(X), \mathrm{Pic}^{+}(X)$ and $\mathrm{Alb}^{-}(X)$ do have the appropriate Hodge, De Rham and $\ell$-adic realizations (in Sections $2.4-2.6,3.3,4.3-4.5$ and 5.3 respectively). We mostly deal with the geometric case, i.e., we consider varieties $X$ over an algebraically closed field $k$; the case when $k$ is not algebraically closed is considered in Chapter 7.

We show as well that our definitions are functorial and independent of choices of resolutions or compactifications (e.g., see Section 2.3) and depend only on the seminormalization of the given variety (see Section 6.1). We remark (in Section 6.2) that $\mathrm{Alb}^{+}$can be contravariant functorial only for morphisms between varieties of the same dimension, and similarly $\mathrm{Pic}^{-}$is covariant functorial for such maps. We then show the homotopical invariance of $\mathrm{Pic}^{+}$(and hence dually of $\mathrm{Alb}^{-}$), and that $\mathrm{Pic}^{-}$and $\mathrm{Pic}^{+}$ (and dually, the corresponding Albanese 1-motives) are invariant under formation of projective bundles (see Section 6.3).

For proper $X$, we remark that our "cohomological" Albanese 1-motive $\mathrm{Alb}^{+}(X)$ is a quotient of Serre's Albanese of the regular locus $X_{\text {reg }}$, i.e., we have an extension

$$
0 \rightarrow T(S) \rightarrow \mathrm{Alb}^{-}\left(X_{\text {reg }}\right) \rightarrow \mathrm{Alb}^{+}(X) \rightarrow 0
$$

where $T(S)$ is a torus whose character group is a sublattice of the lattice of Weil divisors which are supported on the singular locus $S$. Thus, if $X$ is also irreducible and normal, then $T(S)=0$, and further, any non-zero Cartier divisor supported on the exceptional locus of a resolution is not numerically equivalent to zero; therefore, $\mathrm{Alb}^{-}\left(X_{\mathrm{reg}}\right)=\mathrm{Alb}^{+}(X)$ is an abelian variety which is isomorphic to the Albanese variety $\operatorname{Alb}(\tilde{X})$ of any resolution of singularities $\widetilde{X}$ of $X$. In general, $\mathrm{Alb}^{-}\left(X_{\mathrm{reg}}\right)$ is a torus bundle over $\operatorname{Alb}(\tilde{X})$ whose pull-back to $X_{\text {reg }}$ (under a suitable Albanese 
mapping) is canonically trivialized. Thus, after choosing appropriate base points, there is a (canonical) section

$$
a^{-}: X_{\text {reg }} \rightarrow \operatorname{Alb}^{-}\left(X_{\text {reg }}\right)
$$

which is a universal morphism to semi-abelian varieties in the sense of Serre [47] (see Section 5.2).

We then show (in Section 6.4) that $a^{-}$factors through rational equivalence yielding a "motivic" Abel-Jacobi mapping

$$
a^{+}: \mathrm{CH}^{n}(X)_{\operatorname{deg} 0} \rightarrow \mathrm{Alb}^{+}(X)
$$

from the Levine-Weibel "cohomological" Chow group [30] of zero-cycles on a projective variety $X$. We also prove that $a^{+}$is the universal regular homomorphism to semiabelian varieties (compare with $[\mathbf{1 9}]$ and $[\mathbf{8}]$ ). By using this universal property we get (in Section 6.5) "motivic" Gysin maps for projective local complete intersection morphisms, i.e., for such a morphism $g: X^{\prime} \rightarrow X$ we get a push-forward

$$
g_{*}^{+}: \operatorname{Alb}^{+}\left(X^{\prime}\right) \rightarrow \operatorname{Alb}^{+}(X)
$$

and, dually, a pull-back $g_{-}^{*}: \mathrm{Pic}^{-}(X) \rightarrow \mathrm{Pic}^{-}\left(X^{\prime}\right)$.

We note that the isogeny classes of our 1-motives define objects in the triangulated category of mixed motives of Voevodsky, since it contains Deligne's 1-motives (tensor $\mathbb{Q})$. Therefore we can view our constructions as determining "Picard and Albanese mixed motives" as well.

We finally remark that the work presented in this volume was done a few years ago; in fact, these results were previously announced in a short note [6].

\subsection{Some further questions}

We expect purely algebraic proofs for the Lefschetz theorem on inclusions of general complete intersections $g: Y \hookrightarrow X$ (i.e., $g_{*}^{+}$and $g_{-}^{*}$ would be isomorphisms in this case, if $\operatorname{dim} Y \geqslant 2$ ) as well as Roitman theorems on torsion zero-cycles (i.e., $a^{+}$ would be an isomorphism on torsion, see $[\mathbf{2}]$ and $[\mathbf{8}]$ for the case $k=\mathbb{C}$, and [46] for the homological case): these matters are of independent interest, and we hope to treat them elsewhere. In the context of algebraic proofs, it seems desirable as well to have such a proof that $\mathrm{Pic}^{+}(X)$ and $\mathrm{Alb}^{-}(X)$ are independent of the choices of hypercovering and compactification (see Remark 4.4.4). In fact, the underlying philosophy of the theory of 1-motives suggests that it should be possible (or at least desirable) to obtain all constructions and properties "intrinsically", without recourse to the use of any specific realization functor. From this point of view, another problem is to prove "directly" that the Gysin maps for projective local complete intersection morphisms are independent of the factorization (see Remark 6.5.3).

It is natural to ask whether there is an analogue of our results in positive characteristic. After the work of de Jong, there are smooth proper hypercoverings in this 
context as well, which suggests that one could possibly extend the definitions of $\mathrm{Pic}^{+}$ and $\mathrm{Alb}^{-}$to this case. However, since our definitions of $\mathrm{Pic}^{-}$and $\mathrm{Alb}^{+}$, and the proofs that they have the correct realizations, make use of resolution of singularities and duality theory, it is not clear to us how these might extend to positive characteristics. In positive characteristics, one also needs to better understand what would play the role of the De Rham realization.

More generally, since Deligne has defined a notion of a 1-motive over a base scheme $S$, we could ask for the appropriate families $X \rightarrow S$ for which it is possible to define $\mathrm{Pic}^{+}(X / S), \mathrm{Pic}^{-}(X / S)$, and the corresponding Albanese 1-motives, as 1-motives over $S$. Going still further, one could speculate about possible analogous 1-motives in the context of Arakelov geometry.

\subsection{Notations}

We are mainly concerned with schemes locally of finite type over a base field $k$ of characteristic zero, which is assumed to be algebraically closed in most of this work; we will consider non algebraically closed fields in Chapter 7 . We tacitly assume that our schemes are reduced and separated, unless explicitly mentioned otherwise. A variety will be a reduced, separated $k$-scheme of finite type. We will often tacitly identify a variety over $k=\bar{k}$ with its set of closed points. The hypothesis of zero characteristic is repeatedly used, often without explicit mention, for example via the existence of resolutions of singularities.

We denote by $X$. a simplicial $k$-scheme, whose components $X_{i}$ are $k$-schemes, and we denote by $d_{j}^{i}: X_{i} \rightarrow X_{i-1}, 0 \leqslant j \leqslant i$, the face maps; we omit upper indices if there is no risk of misunderstanding, e.g., we may write $d_{0}$ and $d_{1}$ for the two faces map from $X_{1}$ to $X_{0}$. We will also sometimes identify a $k$-scheme $X$ with the "constant" simplicial scheme $X$. it defines, where $X_{n}=X$ for all $n \geqslant 0$, and all face and degeneracy morphisms are the identity; if $\pi: X . \rightarrow X$ is the augmentation, then we note that for any sheaf of abelian groups $\mathcal{F}$ on $X$, the canonical map $\mathcal{F} \rightarrow \mathbf{R} \pi_{*}\left(\pi^{*} \mathcal{F}\right)$ is an isomorphism, and we have canonical isomorphisms $H^{*}(X, \mathcal{F}) \cong \mathbb{H}^{*}\left(X ., \pi^{*} \mathcal{F}\right)$.

For a $\mathbb{C}$-variety $X$ we will denote by $H^{*}(X, \mathbb{Z}(\cdot))$ (resp. $\left.H_{*}(X, \mathbb{Z}(\cdot))\right)$ the singular cohomology (resp. homology) group of the associated analytic space as well as the (Tate twisted) mixed Hodge structure on it. Concerning mixed Hodge structures we will use Deligne's notation [15]: in particular, we will denote by $W_{i} H$ the weight filtration on $H_{\mathbb{Q}}$ (and if $H$ is torsion free, on $H_{\mathbb{Z}}$ as well), and by $F^{i} H$ the Hodge filtration on $H_{\mathbb{C}}$.

For a simplicial scheme $X$. and a simplicial abelian sheaf $\mathcal{F}_{X}$. we will denote by $\mathbb{H}^{*}\left(X ., \mathcal{F}_{X}.\right)$ the cohomology groups obtained from the right derived functor of the following left exact functor

$$
\mathcal{F}_{X} . \longmapsto \operatorname{ker}\left(\Gamma\left(X_{0}, \mathcal{F}_{X_{0}}\right) \stackrel{d_{0}^{*}-d_{1}^{*}}{\longrightarrow} \Gamma\left(X_{1}, \mathcal{F}_{X_{1}}\right)\right) .
$$


The same conventions as above apply to simplicial $\mathbb{C}$-schemes $X$. and the mixed Hodge structure on $\mathbb{H}^{*}(X ., \mathbb{Z}(\cdot))$.

We denote duals by $(-)^{\vee}$ with the following conventions: if $G$ is a group scheme of additive type then $G^{\vee}$ is $\operatorname{Hom}\left(G, \mathbb{G}_{a}\right)$; if $G$ is a torus or it is locally constant and torsion free then $G^{\vee}$ is $\operatorname{Hom}\left(G, \mathbb{G}_{m}\right)$; if $A$ is an abelian variety then $A^{\vee}$ is $\operatorname{Pic}^{0}(A)$; if $H$ is a mixed Hodge structure then $H^{\vee}$ is the internal $\operatorname{Hom}(H, \mathbb{Z}(1))$.

We denote by $\operatorname{Div}(X)$ the group of Weil divisors on an equidimensional variety $X$. If $Y, Z$ are closed subschemes of $X$, we denote by $\operatorname{Div}_{Z}(X) \subset \operatorname{Div}(X)$ the subgroup of divisors which are supported on $Z$, and by $\operatorname{Div}(X, Y) \subset \operatorname{Div}(X)$ the subgroup of divisors which have the support disjoint from $Y$; finally set

$$
\operatorname{Div}_{Z}(X, Y) \stackrel{\text { def }}{=} \operatorname{Div}_{Z}(X) \cap \operatorname{Div}(X, Y) .
$$

For any (possibly singular) variety $X$ we let denote by $\mathrm{CH}_{d}(X)$ the "homological" (Fulton) Chow groups [21] of $d$-dimensional cycles on $X$. We denote by $\mathrm{CH}^{n}(X)$ the "cohomological" (Levine-Weibel) Chow group [30] of zero-cycles supported on the regular locus of an $n$-dimensional quasi-projective variety $X$ over an algebraically closed field.

If $f: G_{1} \rightarrow G_{2}$ is a homomorphism of $k$-group schemes, $\operatorname{ker}^{0} f$ will denote the identity component of the kernel of $f$. 



\section{CHAPTER 1}

\section{PRELIMINARIES ON 1-MOTIVES}

For the sake of exposition, and to fix notation and terminology, we collect some general facts concerning 1-motives.

\subsection{Deligne's definition}

Let $S$ be any scheme. We will denote by $M=(L, A, T, G, u)$ a 1-motive over $S$, i.e., an extension $G$ of an abelian scheme $A$ by a torus $T$ over $S$, a group scheme $L$ which is, locally for the étale topology on $S$, isomorphic to a finitely-generated free abelian constant group, and an $S$-homomorphism $L \stackrel{u}{\rightarrow} G$ (see [15, § 10]).

Diagrammatically a 1 -motive $M=(L, A, T, G, u)$ can be represented as

$$
\begin{aligned}
& L \\
& \downarrow u \\
1 \rightarrow T \rightarrow & G \rightarrow A \rightarrow 0
\end{aligned}
$$

and can be regarded also as defining a complex of group schemes $M=[L \stackrel{u}{\rightarrow} G]$, where $L$ is in degree -1 and $G$ is in degree 0 .

A group scheme $G$ which is an extension of an abelian scheme $A$ by a torus $T$ is also usually called a semi-abelian scheme, and we are not going to distinguish it from the 1-motive which it defines in a canonical way (i.e., by taking $L$ to be zero). The same convention applies to the case of an abelian variety $A$ (identified with the 1 motive $(0, A, 0, A, 0))$ or a torus. A lattice $L$ determines a 1 -motive $[L \rightarrow 0]$, which we denote by $L[1]$ (consistent with the notation when considered as a complex of group schemes).

A morphism of 1-motives is a morphism of the corresponding complexes of group schemes. Moreover, there is a natural full embedding of the category of 1-motives over $S$ into the derived category of bounded complexes of sheaves for the fppf-topology on $S$ (cf. [43, Prop.2.3.1]). 
A 1-motive $M$ is canonically equipped with an increasing filtration by sub-1-motives as follows:

$$
W_{i}(M)= \begin{cases}M & i \geqslant 0 \\ G & i=-1 \\ T & i=-2 \\ 0 & i \leqslant-3\end{cases}
$$

In particular we have $\operatorname{gr}_{-1}^{W}(M)=A$.

A complex of 1-motives is exact if it determines an exact sequence of complexes of group schemes. For example, associated to any 1-motive $M=(L, A, T, G, u)$ there is a functorial exact sequence of 1-motives

$$
0 \rightarrow G \rightarrow M \rightarrow L[1] \rightarrow 0
$$

where $L[1]=\operatorname{gr}_{0}^{W}(M)=[L \rightarrow 0]$.

\subsection{Hodge realization}

We recall that the Hodge realization $T_{\text {Hodge }}(M)(T(M)$ for short) of a 1-motive $M$ over $k=\mathbb{C}$ (see the construction by Deligne in $[\mathbf{1 5}, 10.1 .3]$ ) is the mixed Hodge structure given by the lattice $T_{\mathbb{Z}}(M)$ obtained by the pull-back of $u: L \rightarrow G$ along $\exp : \operatorname{Lie}(G) \rightarrow G$, with the weight filtration

$$
W_{i} T(M) \stackrel{\text { def }}{=} \begin{cases}T_{\mathbb{Z}}(M) & i \geqslant 0 \\ H_{1}(G) & i=-1 \\ H_{1}(T) & i=-2 \\ 0 & i \leqslant-3\end{cases}
$$

The Hodge filtration is defined by

$$
F^{0}\left(T_{\mathbb{Z}}(M) \otimes \mathbb{C}\right) \stackrel{\text { def }}{=} \operatorname{ker}\left(T_{\mathbb{Z}}(M) \otimes \mathbb{C} \rightarrow \operatorname{Lie}(G)\right),
$$

whence $\operatorname{gr}_{-1}^{W} T(M) \cong H_{1}(A, \mathbb{Z})$ as pure Hodge structures of weight -1 . The functor

$$
M \longmapsto T_{\text {Hodge }}(M)
$$

is an equivalence between the category of 1-motives and the full subcategory of torsion free $\mathbb{Z}$-mixed Hodge structures of type

$$
\{(0,0),(0,-1),(-1,0),(-1,-1)\}
$$

such that $\operatorname{gr}_{-1}^{W}(H)$ is polarizable. In fact, Deligne $([\mathbf{1 5}, \S 10.1 .3])$ observed that such a torsion free mixed Hodge structure $H$ yields ( $i$ ) an abelian variety $A$ with

$$
A(\mathbb{C})=\frac{\operatorname{gr}_{-1}^{W}\left(H_{\mathbb{C}}\right)}{H_{\mathbb{Z}}+F^{0} \operatorname{gr}_{-1}^{W}\left(H_{\mathbb{C}}\right)}
$$


(ii) an algebraic torus $T$ with character group $\operatorname{gr}_{-2}^{W}\left(H_{\mathbb{Z}}\right)$, so that

$$
T(\mathbb{C})=\operatorname{Hom}\left(\mathrm{gr}_{-2}^{W}\left(H_{\mathbb{Z}}\right), \mathbb{C}^{*}\right)
$$

and (iii) a complex algebraic group $G$ with

$$
G(\mathbb{C})=\frac{W_{-1}\left(H_{\mathbb{C}}\right)}{W_{-1}\left(H_{\mathbb{Z}}\right)+F^{0} \cap W_{-1}\left(H_{\mathbb{C}}\right)}
$$

which is an algebraic extension of $A$ by $T$; moreover, the canonical map $H_{\mathbb{Z}} \rightarrow H_{\mathbb{C}}$ yields (iv) a homomorphism $u$ from the lattice $L=\operatorname{gr}_{0}^{W}\left(H_{\mathbb{Z}}\right)$ to the group $G(\mathbb{C})$. Deligne considered such a set of data (i) - (iv) as defining a 1-motive over $\mathbb{C}$, and showed that it is equivalent to the given mixed Hodge structure.

Thus any 1-motive $M=(L, A, T, G, u)$ over $\mathbb{C}$ has a Hodge realization $T_{\text {Hodge }}(M)$ and, conversely, any such mixed Hodge structure yields, canonically, a 1-motive. The exact sequence (1) gives rise to an exact sequence of Hodge realizations

$$
0 \rightarrow T_{\text {Hodge }}(G) \rightarrow T_{\text {Hodge }}(M) \rightarrow T_{\text {Hodge }}(L[1]) \rightarrow 0
$$

For example, any abelian variety $A$ over $\mathbb{C}$ considered as a 1-motive (i.e., we regard $A$ as $(0, A, 0, A, 0))$, has Hodge realization $H_{1}(A, \mathbb{Z})$; in particular, for a non-singular complete variety $X$ over $\mathbb{C}$, the classical Albanese variety $M=\operatorname{Alb}(X)$ has Hodge realization $(n=\operatorname{dim} X)$

$$
T(M)=H_{1}(\operatorname{Alb}(X), \mathbb{Z}) \cong H_{1}(X, \mathbb{Z}) /(\text { torsion }) \cong H^{2 n-1}(X, \mathbb{Z}(n)) /(\text { torsion })
$$

because of the canonical isomorphism $\operatorname{Alb}(X) \cong J^{n}(X)$ where $J^{n}(X)$ is the cohomological (Griffiths) intermediate jacobian; for a smooth complete variety the Hodge structures on $H_{1}(X, \mathbb{Z})$ and $H^{2 n-1}(X, \mathbb{Z}(n))$ are canonically isomorphic (by Poincaré duality) and they both correspond to the Albanese variety.

\section{3. $\ell$-adic and étale realization}

Let $M=[L \stackrel{u}{\rightarrow} G]$ be a 1-motive over $S$ which we consider as a complex of fppfsheaves over $S$ with $L$ in degree -1 and $G$ in degree 0 . For any fixed integer $m$ we let $T_{\mathbb{Z} / m}(M)$ be $H^{-1}(M / m)$ where $M / m$ is the cone of multiplication by $m$ on $M$. Then $T_{\mathbb{Z} / m}(M)$ is a finite group scheme which is flat over $S$, and is étale if $S$ is defined over $\mathbb{Z}\left[\frac{1}{m}\right]$.

For $S=\operatorname{Spec}(k)$ and $k=\bar{k}$ we then have

$$
T_{\mathbb{Z} / m}(M)(k)=\frac{\{(x, g) \in L \times G(k) \mid u(x)=-m g\}}{\{(m x,-u(x)) \mid x \in L\}}
$$

If $\ell$ is a prime number then the $\ell$-adic realization $T_{\ell}(M)$ is simply defined to be the inverse limit over $\nu$ of $T_{\mathbb{Z} / \ell^{\nu}}(M) . T_{\ell}(M)$ is the $\ell$-adic Tate module of an $\ell$-divisible group. The $\ell$-adic realization of an abelian variety $A$ is the $\ell$-adic Tate module of $A$; the $\ell$-adic realization of a lattice $L$ is $L \otimes_{\mathbb{Z}} \mathbb{Z}_{\ell}$. 
If $S=\operatorname{Spec}(k)$ and $k=\bar{k}$ is of characteristic zero then

$$
\widehat{T}(M) \stackrel{\text { def }}{=}{\underset{m}{m}}_{\lim _{\mathbb{Z} / m}}(M)=\prod_{\ell} T_{\ell}(M) .
$$

We call $\widehat{T}(M)$ the étale realization of $M$. In particular, if $k=\mathbb{C}$ then $\widehat{T}(M)=$ $T_{\mathbb{Z}}(M) \otimes \widehat{\mathbb{Z}}$ because the complex $T_{\mathbb{Z}}(M) \rightarrow$ Lie $G$ is quasi-isomorphic to $M(\mathbb{C})$, and therefore $M / m(\mathbb{C})$ is quasi-isomorphic to $\left(T_{\mathbb{Z}}(M) \otimes \mathbb{Z} / m\right)[+1]$.

The exact sequence (1) of 1-motives yields a long exact sequence of cohomology groups

$$
H^{-2}(L[1] / m) \rightarrow H^{-1}(G / m) \rightarrow H^{-1}(M / m) \rightarrow H^{-1}(L[1] / m) \rightarrow H^{0}(G / m)
$$

where $H^{-2}(L[1] / m)=\operatorname{ker}(L \stackrel{m}{\longrightarrow} L)$ is clearly zero, and $H^{0}(G / m)=\operatorname{coker}(G \stackrel{m}{\longrightarrow} G)$ vanishes since multiplication by $m$ is an epimorphism of fppf-sheaves. In the sequence above we are left with finite group schemes, and thus, by taking the inverse limit on $m$, the sequence yields the following short exact sequence

$$
0 \rightarrow \widehat{T}(G) \rightarrow \widehat{T}(M) \rightarrow \widehat{T}(L[1]) \rightarrow 0
$$

The exact sequence above is clearly functorial with respect to maps of 1-motives, since it is obtained from (1) by applying the functor $\widehat{T}$; it is the étale analogue of (2).

We will later make use of the following fact.

Proposition 1.3.1. - The étale realization functor $\widehat{T}$ from the category of 1 motives over $k=\bar{k}$ to abelian groups is faithful, and further, it reflects isomorphisms (i.e., if $M \rightarrow M^{\prime}$ is a map of 1-motives such that $\widehat{T}(M) \cong \widehat{T}\left(M^{\prime}\right)$ then $M \rightarrow M^{\prime}$ is an isomorphism in the category of 1-motives).

Proof. - Consider $M=[L \stackrel{u}{\rightarrow} G], M^{\prime}=\left[L^{\prime} \stackrel{u^{\prime}}{\rightarrow} G^{\prime}\right]$ and $f: M \rightarrow M^{\prime}$. Now $\widehat{T}$ is clearly an additive functor; hence, in order to show that $\widehat{T}$ is faithful, we just need to show that $\widehat{T}(f)=0$ implies $f=0$. By making use of the exact sequence (3) we can see that it is enough to check it separately for maps of semi-abelian schemes or lattices. Since torsion points are Zariski dense in a semi-abelian scheme over $k=\bar{k}$, $\widehat{T}(f)=0$ implies $f=0$ for morphisms $f$ between semi-abelian schemes. Finally $\widehat{T}(L[1])=L \otimes \widehat{\mathbb{Z}}$ which is clearly faithful.

If $M \rightarrow M^{\prime}$ induces an isomorphism $\widehat{T}(M) \cong \widehat{T}\left(M^{\prime}\right)$ then by $(3)$ we have that $\widehat{T}(G)$ injects into $\widehat{T}\left(G^{\prime}\right)$ and $\widehat{T}(L[1])$ surjects onto $\widehat{T}\left(L^{\prime}[1]\right)$, therefore we have an extension of lattices

$$
0 \rightarrow L^{\prime \prime} \rightarrow L \rightarrow L^{\prime} \rightarrow 0
$$

Moreover by the snake lemma applied to the resulting diagram given by (3) we get that

$$
\widehat{T}\left(L^{\prime \prime}[1]\right) \cong \frac{\widehat{T}\left(G^{\prime}\right)}{\widehat{T}(G)}
$$


Now we have that $F=\operatorname{ker}\left(G \rightarrow G^{\prime}\right)$ is a finite group, since $\widehat{T}(G) \hookrightarrow \widehat{T}\left(G^{\prime}\right)$; we can see that

$$
F \cong \frac{\widehat{T}(G / F)}{\widehat{T}(G)} \hookrightarrow \frac{\widehat{T}\left(G^{\prime}\right)}{\widehat{T}(G)} .
$$

Thus $F=0$, since it injects into $\widehat{T}\left(L^{\prime \prime}[1]\right)$ which is torsion free. If we let $G^{\prime \prime}$ denote the quotient of $G$ by $G^{\prime}$, we then get the following exact sequence of complexes

$$
0 \rightarrow\left[L^{\prime \prime} \rightarrow 0\right] \rightarrow[L \rightarrow G] \rightarrow\left[L^{\prime} \rightarrow G^{\prime}\right] \rightarrow\left[0 \rightarrow G^{\prime \prime}\right] \rightarrow 0
$$

Applying $\widehat{T}$ we have that the composition of the following maps

$$
\widehat{T}\left(L^{\prime \prime}[1]\right) \rightarrow \widehat{T}(M) \stackrel{\cong}{\longrightarrow} \widehat{T}\left(M^{\prime}\right) \rightarrow \widehat{T}\left(G^{\prime \prime}\right)
$$

is the zero map as well as an isomorphism, therefore $\widehat{T}\left(L^{\prime \prime}[1]\right)=\widehat{T}\left(G^{\prime \prime}\right)=0$ whence $L^{\prime \prime}=G^{\prime \prime}=0$, i.e., $M \stackrel{\cong}{\cong} M^{\prime}$.

\subsection{De Rham realization}

The De Rham realization of a 1-motive $M=[L \stackrel{u}{\rightarrow} G]$ over an algebraically closed field $k$ is obtained via Grothendieck's interpretation of $H_{\mathrm{DR}}^{1}(c f .[\mathbf{3 2}, \S 4],[\mathbf{1 5}, 10.1 .7]$ and [18]). Consider $\mathbb{G}_{a}$ as a complex of $k$-group schemes concentrated in degree 0 . Then, for any 1-motive $M$ over $k$, we have $\operatorname{Hom}\left(M, \mathbb{G}_{a}\right)=0$, and there is an extension

$$
0 \rightarrow \operatorname{Ext}\left(L[1], \mathbb{G}_{a}\right) \rightarrow \operatorname{Ext}\left(M, \mathbb{G}_{a}\right) \rightarrow \operatorname{Ext}\left(G, \mathbb{G}_{a}\right) \rightarrow 0
$$

where $\operatorname{Ext}\left(G, \mathbb{G}_{a}\right)$ is canonically identified with the Lie algebra of the dual of the abelian variety $A$ (the abelian quotient of the semi-abelian variety $G$ ), and

$$
\operatorname{Ext}\left(L[1], \mathbb{G}_{a}\right)=\operatorname{Hom}\left(L, \mathbb{G}_{a}\right) .
$$

Hence the $k$-vector space $\operatorname{Ext}\left(M, \mathbb{G}_{a}\right)$ is finite dimensional.

By general arguments $(c f .[\mathbf{3 2}],[\mathbf{1 5}]) M$ has a universal $\mathbb{G}_{a}$-extension $M^{\natural}$, in Deligne's notation $[\mathbf{1 5}, 10.1 .7]$, where $M^{\natural}=\left[L \stackrel{u^{\natural}}{\longrightarrow} G^{\natural}\right]$ is a complex of $k$-group schemes which is an extension of $M$ by the vector space $\operatorname{Ext}\left(M, \mathbb{G}_{a}\right)^{\vee}$, considered as a complex in degree zero. In fact, we have a diagram

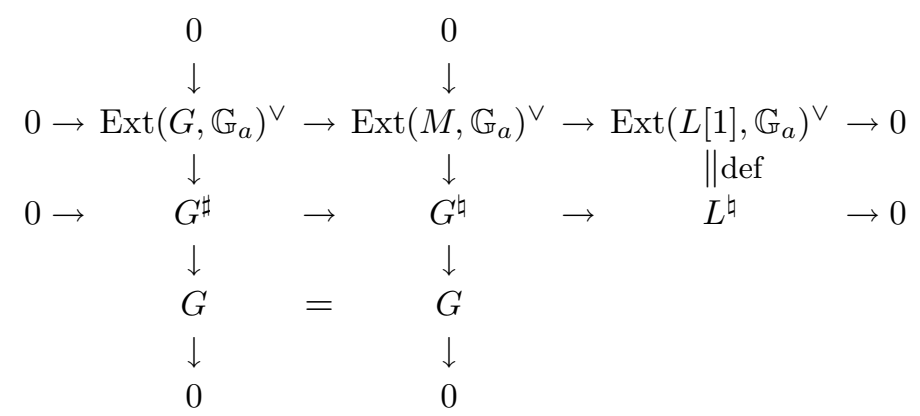


where $G^{\natural}$ is the push-out of the universal $\mathbb{G}_{a}$-extension $G^{\sharp}$ of the semi-abelian variety $G$. The canonical map $u^{\natural}: L \rightarrow G^{\natural}$ is such that the composition

$$
L \stackrel{u^{\natural}}{\longrightarrow} G^{\natural} \rightarrow L^{\natural}=\operatorname{Ext}\left(L[1], \mathbb{G}_{a}\right)^{\vee}=\operatorname{Hom}\left(L, \mathbb{G}_{a}\right)^{\vee}
$$

is the natural evaluation map.

In particular we get the following extension

$$
0 \rightarrow \operatorname{Ext}\left(M, \mathbb{G}_{a}\right)^{\vee} \rightarrow G^{\natural} \rightarrow G \rightarrow 0
$$

of group schemes. The De Rham realization of $M$ is then defined as

$$
T_{\mathrm{DR}}(M) \stackrel{\text { def }}{=} \operatorname{Lie} G^{\natural},
$$

with the Hodge-De Rham filtration given by

$$
F^{0} T_{\mathrm{DR}}(M) \stackrel{\text { def }}{=} \operatorname{ker}\left(\operatorname{Lie} G^{\natural} \rightarrow \operatorname{Lie} G\right) \cong \operatorname{Ext}\left(M, \mathbb{G}_{a}\right)^{\vee} .
$$

If $k=\mathbb{C}$ then the De Rham realization is compatible with the Hodge realization, see $[\mathbf{1 5}, \S 10.1 .8, \S 10.3 .15]$. We also have an exact sequence

$$
0 \rightarrow T_{\mathrm{DR}}(G) \rightarrow T_{\mathrm{DR}}(M) \rightarrow T_{\mathrm{DR}}(L[1]) \rightarrow 0
$$

which is the sequence of Lie algebras associated to

$$
0 \rightarrow G^{\sharp} \rightarrow G^{\natural} \rightarrow L^{\natural} \rightarrow 0 .
$$

We may also view (4) as obtained by applying the functor $T_{\mathrm{DR}}$ to (1); thus (4) is the De Rham version of (2) and (3).

Let $X$ be a smooth projective variety over $k=\bar{k}$ of characteristic 0 , and let $\operatorname{Pic}^{\natural}(X)$ be the group of isomorphism classes of pairs $(\mathcal{L}, \nabla)$ where $\mathcal{L}$ is a line bundle on $X$ and $\nabla$ is an integrable connection on $\mathcal{L}$. Then there is the following extension

$$
0 \rightarrow H^{0}\left(X, \Omega_{X}^{1}\right) \rightarrow \operatorname{Pic}^{\natural}(X)^{0} \rightarrow \operatorname{Pic}^{0}(X) \rightarrow 0
$$

where $\operatorname{Pic}^{\natural}(X)^{0}$ is the the subgroup of those pairs $(\mathcal{L}, \nabla)$ such that $\mathcal{L} \in \operatorname{Pic}^{0}(X)$. The above extension is the group of points of the universal $\mathbb{G}_{a}$-extension of the abelian variety $\operatorname{Pic}_{X / k}^{0}$ and $\operatorname{LiePic}^{\natural}(X)^{0}=H_{\mathrm{DR}}^{1}(X)(1)$ (cf. $\left.[\mathbf{3 2}, \S 4]\right)$, where the twist (1) indicates that the indexing of the Hodge filtration is shifted by 1 . In general, for any abelian variety $A, A^{\natural}=\operatorname{Pic}^{\natural}\left(A^{\vee}\right)^{0}$, so that $A$ has De Rham realization

$$
T_{\mathrm{DR}}(A)=H_{\mathrm{DR}}^{1}(A) \stackrel{\text { def }}{=} H_{1}^{D R}(A) \text {. }
$$

\subsection{Cartier duals}

We now recall briefly the construction by Deligne $[\mathbf{1 5}, \S 10.2 .11-13]$ of the dual 1motive. The definition is motivated by the case of 1 -motives over $\mathbb{C}$ where the Hodge realization has a dual mixed Hodge structure which yields the dual 1-motive. In fact, if $H$ is a torsion free mixed Hodge structure of type $\{(0,0),(0,-1),(-1,0),(-1,-1)\}$ such that $\operatorname{gr}_{-1}^{W}(H)$ is polarizable then $H^{\vee}=\operatorname{Hom}(H, \mathbb{Z}(1))$ is again of the same kind; 
since any 1-motive $M$ over $\mathbb{C}$ corresponds (uniquely up to isomorphism) to such an $H=T_{\text {Hodge }}(M)$ we can just set

$$
T_{\text {Hodge }}\left(M^{\vee}\right)=\left(T_{\text {Hodge }}(M)\right)^{\vee}
$$

as an implicit "analytic" definition for $M^{\vee}$.

In order to give an algebraic description of $M^{\vee}$ the yoga of biextensions is needed: see [34] for the original definition of biextension of formal groups and compare [27, VII, (2.1)] for biextensions of abelian groups in an arbitrary topos. In order to deal with 1-motives we need to consider the following generalization of Grothendieck biextensions due to Deligne $[\mathbf{1 5}, \S 10.2 .1]$.

Let $K_{i}=\left[A_{i} \stackrel{u_{i}}{\longrightarrow} B_{i}\right]$ for $i=1,2$ two complexes of abelian sheaves (on a Grothendieck site) concentrated in degrees -1 and 0 . A biextension of $K_{1}$ and $K_{2}$ by an abelian sheaf $H$ is given by a Grothendieck biextension $P$ of $B_{1}$ and $B_{2}$ by $H$ and a pair of compatible trivializations of the biextensions of $A_{1} \times B_{2}$ and $B_{1} \times A_{2}$ obtained by pullbacks. More precisely, this means one has the following data:

(i) an $H$-torsor $P$ over $B_{1} \times B_{2}$, which is a Grothendieck biextension,

(ii) a biadditive section of the biextension $\left(1 \times u_{2}\right)^{*}(P)$ over $B_{1} \times A_{2}$,

(iii) a biadditive section of the biextension $\left(u_{1} \times 1\right)^{*}(P)$ over $A_{1} \times B_{2}$, along with

(iv) the compatibility condition that the two induced (biadditive) sections $A_{1} \times A_{2} \rightarrow$ $\left(u_{1} \times u_{2}\right)^{*}(P)$ coincide.

Let $\operatorname{Biext}\left(K_{1}, K_{2} ; H\right)$ denote the group of isomorphism classes of biextensions. Now we have the following fundamental results (see $[\mathbf{1 5}, \S 10.2 .3-9]$ for details).

Let $M_{1}$ and $M_{2}$ be two 1-motives over $\mathbb{C}$. Then there is a natural isomorphism

$$
\operatorname{Biext}\left(M_{1}, M_{2} ; \mathbb{G}_{m}\right) \cong \operatorname{Hom}\left(T_{\text {Hodge }}\left(M_{1}\right) \otimes T_{\text {Hodge }}\left(M_{2}\right), \mathbb{Z}(1)\right)
$$

where the Hom is taken in the abelian category of mixed Hodge structures. Similarly, there are purely algebraic versions of this correspondence (over $k=\bar{k}$ of characteristic 0 ) which associates to a given biextension $P \in \operatorname{Biext}\left(M_{1}, M_{2} ; \mathbb{G}_{m}\right)$ the following pairings

$$
T_{\mathbb{Z} / m}\left(M_{1}\right) \otimes T_{\mathbb{Z} / m}\left(M_{2}\right) \rightarrow \mu_{m}
$$

and

$$
T_{\mathrm{DR}}\left(M_{1}\right) \otimes T_{\mathrm{DR}}\left(M_{2}\right) \rightarrow k(1) .
$$

Here $k(1)$ is a 1 -dimensional filtered $k$-vector space with filtration $F^{-1} k(1)=k(1)$ and $F^{0} k(1)=0$. The latter pairing on De Rham realizations is obtained by pulling back $P$ to a $\natural$-biextension $P^{\natural}$ of $M_{1}^{\natural}$ and $M_{2}^{\natural}$ by $\mathbb{G}_{m}$. Over $\mathbb{C}$, these latter two pairings on the realizations are obtained from the former Hodge theoretic one by reduction modulo $m$ and by taking the associated $\mathbb{C}$-pairing, respectively.

Let $M$ be a 1-motive over $\mathbb{C}$. Then, by the above, the canonical pairing

$$
T_{\text {Hodge }}(M) \otimes T_{\text {Hodge }}(M)^{\vee} \rightarrow \mathbb{Z}(1)
$$


defines a biextension $\mathcal{P}$ of $M$ and $M^{\vee}$ by $\mathbb{G}_{m}$, which is called the Poincaré biextension of $M$ and $M^{\vee}$. This construction can be made algebraic. Let $M=[L \stackrel{u}{\longrightarrow} G]$ be a 1-motive over an algebraically closed field, i.e.,

$$
\begin{aligned}
L & \\
& \downarrow u \\
1 \rightarrow T \rightarrow & G \rightarrow A \rightarrow 0
\end{aligned}
$$

where $G$ is an extension of an abelian variety $A$ by a torus $T$.

Assume $T=1$ first. Then there is an extension

$$
1 \rightarrow \operatorname{Ext}\left(L[1], \mathbb{G}_{m}\right) \rightarrow \operatorname{Ext}\left(M, \mathbb{G}_{m}\right) \rightarrow \operatorname{Ext}\left(A, \mathbb{G}_{m}\right) \rightarrow 0
$$

where $\operatorname{Ext}\left(L[1], \mathbb{G}_{m}\right)=\operatorname{Hom}\left(L, \mathbb{G}_{m}\right)=L^{\vee}$ is a torus and the dual abelian variety $A^{\vee}=\operatorname{Pic}^{0}(A)$ can be regarded as $\operatorname{Ext}\left(A, \mathbb{G}_{m}\right)(c f .[\mathbf{3 8}],[\mathbf{4 9}])$. Moreover, $\operatorname{Ext}\left(M, \mathbb{G}_{m}\right)$ consists of extensions of $A$ by $\mathbb{G}_{m}$ together with a trivialization of the pull-back on $L$. Let $G^{u}$ denote the group scheme which represents the functor associated to $\operatorname{Ext}\left(M, \mathbb{G}_{m}\right)$ (see $\left.[\mathbf{1 5}, \S 10.2 .11]\right)$. Define $M^{\vee} \stackrel{\text { def }}{=}\left[0 \rightarrow G^{u}\right]$ with the biextension $\mathcal{P}$ of $M$ and $M^{\vee}$ by $\mathbb{G}_{m}$, induced by the "classical" Poincaré biextension (see [34]) of $A$ and $A^{\vee}$ by $\mathbb{G}_{m}$.

On the other hand, if $L=0$, then $T^{\vee}=\operatorname{Hom}\left(T, \mathbb{G}_{m}\right)$ and there is a canonical homomorphism

$$
v: T^{\vee} \rightarrow A^{\vee}
$$

by pushing out characters $\chi: T \rightarrow \mathbb{G}_{m}$ along the given extension $G \in \operatorname{Ext}(A, T)$. Define $M^{\vee} \stackrel{\text { def }}{=}\left[T^{\vee} \stackrel{v}{\rightarrow} A^{\vee}\right]$ with the Poincaré biextension similarly obtained as above. Note that the trivialization is given by $\psi$ in the following push-out diagram

$$
\begin{aligned}
1 & \rightarrow T \rightarrow G \rightarrow A \rightarrow 0 \\
& \chi \downarrow \\
1 & \rightarrow \mathbb{G}_{m} \rightarrow \chi_{*} G \rightarrow A \rightarrow 0,
\end{aligned}
$$

i.e., the biadditive homomorphism $G \times T^{\vee} \rightarrow(1 \times v)^{*} \mathcal{P}$ is defined by $(g, \chi) \mapsto \psi(g) \in$ $\chi_{*} G=\mathcal{P}_{(g, v(\chi))}$, where $\mathcal{P}$ is the pull-back of the "classical" Poincaré biextension. We also clearly have that $\left(M^{\vee}\right)^{\vee}=M$ so far.

In general, since $\operatorname{Hom}(L, A) \cong \operatorname{Ext}\left(A^{\vee}, L^{\vee}\right)$, the composite homomorphism

$$
L \stackrel{u}{\longrightarrow} G \rightarrow A
$$

yields an extension $G^{u} \in \operatorname{Ext}\left(A^{\vee}, L^{\vee}\right)$. From the above we get a biextension of $M / W_{-2} M=[L \rightarrow A]$ and $\left(M / W_{-2} M\right)^{\vee}=\left[0 \rightarrow G^{u}\right]$ by $\mathbb{G}_{m}$. Pulling back we then obtain a biextension $\mathcal{P}$ of $M$ and $\left(M / W_{-2} M\right)^{\vee}$ by $\mathbb{G}_{m}$. Moreover, the standard extension $M$ of $M / W_{-2} M$ by $W_{-2} M=[0 \rightarrow T]$ yields the following boundary map

$$
u^{\vee}: T^{\vee}=\operatorname{Hom}\left(W_{-2} M, \mathbb{G}_{m}\right) \rightarrow \operatorname{Ext}\left(M / W_{-2} M, \mathbb{G}_{m}\right)=G^{u}(k)
$$

by pushing out characters. Define

$$
M^{\vee} \stackrel{\text { def }}{=}\left[T^{\vee} \stackrel{u^{\vee}}{\longrightarrow} G^{u}\right]
$$


to be the Cartier dual of $M$. Note that $u^{\vee}$ is a lift of $v: T^{\vee} \rightarrow A^{\vee}$ obtained as above. Thus the biextension $\mathcal{P}$ of $M$ and $\left(M / W_{-2} M\right)^{\vee}$ is trivialized on $L$ and $T^{\vee}$, yielding the desired Poincaré biextension of $M$ and $M^{\vee}$.

Summarizing, the Cartier dual $M^{\vee}$ is given by

$$
\begin{aligned}
& T^{\vee} \\
& \downarrow u^{\vee} \\
1 \rightarrow L^{\vee} \rightarrow & G^{u} \rightarrow A^{\vee} \rightarrow 0,
\end{aligned}
$$

we have that $\left(M^{\vee}\right)^{\vee}=M$ and

$$
\left(\mathrm{gr}_{-i}^{W} M\right)^{\vee}=\operatorname{gr}_{i-2}^{W} M^{\vee}
$$

We also clearly have an exact sequence (according to (1))

$$
0 \rightarrow G^{u} \rightarrow M^{\vee} \rightarrow T^{\vee}[1] \rightarrow 0
$$

which is the Cartier dual of the extension $M$ of $M / W_{-2} M$ by $W_{-2} M$.

By construction, the biextension $\mathcal{P}$ is the pull-back of the "classical" Poincaré biextension of $A$ and $A^{\vee}$ by $\mathbb{G}_{m}$ and it becomes trivial on $L \times T^{\vee}$, i.e., there is a biadditive homomorphism

$$
\psi: L \times T^{\vee} \rightarrow(u \times v)^{*} \mathcal{P} .
$$

The object $\left(L, T^{\vee}, A, A^{\vee}, u, v, \psi\right)$ is then sometimes called the "symmetric avatar" of the 1-motive $M$; the symmetric avatar of the Cartier dual is $\left(T^{\vee}, L, A^{\vee}, A, v, u, \psi^{t}\right)$.

Finally, as recalled above (and proved by Deligne $[\mathbf{1 5}, \S 10.2]$ ) the Poincaré biextension yields pairings on realizations

$$
T_{\mathbb{Z} / m}(M) \otimes T_{\mathbb{Z} / m}\left(M^{\vee}\right) \rightarrow \mu_{m}
$$

and

$$
T_{\mathrm{DR}}(M) \otimes T_{\mathrm{DR}}\left(M^{\vee}\right) \rightarrow k(1)
$$

which are compatible, over the complex numbers, with the canonical pairing induced by the duality between mixed Hodge structures. We therefore can see any given realization of the Cartier dual as being the appropriate dual of that realization of the original 1-motive.

For example, if $X$ is a smooth proper $k$-scheme, then $\mathrm{Pic}_{X / k}^{0}$ is an abelian variety and $\left(\operatorname{Pic}_{X / k}^{0}\right)^{\vee}=\operatorname{Alb}_{X / k}$ is the Albanese variety. Over $k=\mathbb{C}$ we have that $J^{1}(X) \cong$ $\operatorname{Pic}_{X / \mathbb{C}}^{0}(\mathbb{C})$ thus $T_{\text {Hodge }}\left(\operatorname{Pic}_{X / \mathbb{C}}^{0}\right)=H^{1}(X, \mathbb{Z}(1)) \cong H_{2 n-1}(X, \mathbb{Z}(1-n))$, by Poincaré duality, and modulo torison, $T_{\text {Hodge }}\left(\operatorname{Alb}_{X / \mathbb{C}}\right)=H_{1}(X, \mathbb{Z}) \cong H^{2 n-1}(X, \mathbb{Z}(n))$. 



\section{CHAPTER 2}

\section{HOMOLOGICAL PICARD 1-MOTIVE: Pic ${ }^{-}$}

We first begin by introducing some notation and terminology needed below. Let $X$ be an equidimensional variety over a field $k$ of characteristic zero (not necessarily algebraically closed). Let $S \subset X$ be the singular locus and let $f: \widetilde{X} \rightarrow X$ be a resolution of singularities. We let $\widetilde{S}=f^{-1}(S)$ be the reduced inverse image. Consider a smooth compactification of $\tilde{X}$, which we denote by $\bar{X}$; let $Y=\bar{X}-\tilde{X}$ be the boundary, which we assume to be a divisor in $\bar{X}$. Let $\bar{S}$ denote the Zariski closure of $\widetilde{S}$ in $\bar{X}$. We can arrange that the resolution $\widetilde{X}$ and compactification $\bar{X}$ are chosen so that $\bar{X}$ is projective, and $\bar{S}+Y$ is a reduced normal crossing divisor in $\bar{X}$; we call such a compactification $\bar{X}$ a good normal crossing compactification (or good n.c. compactification) of the resolution of $X$. For such a compactification to exist, the resolution $f: \widetilde{X} \rightarrow X$ must be chosen such that $\widetilde{S}$ is a normal crossing divisor.

\subsection{Relative Picard functor}

Associated to any pair $(V, Z)$ consisting of any $k$-scheme $V$ and a closed subscheme $Z$, we have a natural long exact sequence

$$
\cdots \rightarrow H^{0}\left(V, \mathcal{O}_{V}^{*}\right) \rightarrow H^{0}\left(Z, \mathcal{O}_{Z}^{*}\right) \rightarrow \operatorname{Pic}(V, Z) \rightarrow \operatorname{Pic}(V) \rightarrow \operatorname{Pic}(Z) \rightarrow \cdots
$$

induced by the surjection of Zariski (or étale) sheaves $\mathbb{G}_{m, V} \rightarrow i_{*} \mathbb{G}_{m, Z}$ where we denote $i: Z \hookrightarrow V$ the inclusion; here

$$
\operatorname{Pic}(V, Z)=\mathbb{H}^{1}\left(V, \mathbb{G}_{m, V} \rightarrow i_{*} \mathbb{G}_{m, Z}\right)
$$

is the group of isomorphism classes of pairs $(\mathcal{L}, \varphi)$ such that $\mathcal{L}$ is a line bundle on $V$ and $\varphi:\left.\mathcal{L}\right|_{Z} \cong \mathcal{O}_{Z}$ is a trivialization on $Z$ (cf. [52], [42, $\left.\left.\S 2\right],[\mathbf{1 1}, \S 8]\right)$.

Now let $X$ be an equidimensional $k$-variety, and $\bar{X}$ a good normal crossing compactification of a resolution of $X$, with boundary $Y$. 
Lemma 2.1.1. - Let $(\bar{X}, Y)$ be as above. The fpqc-sheaf associated to the relative Picard functor

$$
T \longmapsto \operatorname{Pic}\left(\bar{X} \times_{k} T, Y \times_{k} T\right)
$$

is representable by a k-group scheme which is locally of finite type over $k$. If $k$ is algebraically closed, its group of $k$-points is $\operatorname{Pic}(\bar{X}, Y)$.

Proof. - See the Appendix .

Now assume $k=\bar{k}$. Let $Y=\cup Y_{i}$, where $Y_{i}$ are the (smooth) irreducible components of $Y$.

Proposition 2.1.2. - The sequence (5) yields a semi-abelian group scheme $\operatorname{Pic}^{0}(\bar{X}, Y)$ over $k=\bar{k}$, which can be represented as an extension

$$
1 \rightarrow T(\bar{X}, Y) \rightarrow \operatorname{Pic}^{0}(\bar{X}, Y) \rightarrow A(\bar{X}, Y) \rightarrow 0
$$

where:

(i) $\operatorname{Pic}^{0}(\bar{X}, Y)$ is the connected component of the identity of $\operatorname{Pic}(\bar{X}, Y)$;

(ii) $T(\bar{X}, Y)$ is the $k$-torus

$$
T(\bar{X}, Y) \stackrel{\text { def }}{=} \operatorname{coker}\left(\left(\pi_{\bar{X}}\right)_{*} \mathbb{G}_{m, \bar{X}} \rightarrow\left(\pi_{Y}\right)_{*} \mathbb{G}_{m, Y}\right)
$$

where $\pi_{\bar{X}}: \bar{X} \rightarrow \operatorname{Spec} k, \pi_{Y}: Y \rightarrow$ Spec $k$ are the structure morphisms;

(iii) $A(\bar{X}, Y)$ is the abelian variety

$$
A(\bar{X}, Y) \stackrel{\text { def }}{=} \operatorname{ker}^{0}\left(\operatorname{Pic}^{0}(\bar{X}) \rightarrow \oplus \operatorname{Pic}^{0}\left(Y_{i}\right)\right)
$$

which is the connected component of the identity of the kernel.

Proof. - Everything follows from Lemma 2.1.1 combined with (5), by taking the connected components of the identity, once we know the following.

$$
\operatorname{ker}^{0}\left(\operatorname{Pic}^{0}(\bar{X}) \rightarrow \operatorname{Pic}^{0}(Y)\right)=\operatorname{ker}^{0}\left(\operatorname{Pic}^{0}(\bar{X}) \rightarrow \oplus \operatorname{Pic}^{0}\left(Y_{i}\right)\right) .
$$

Recall that $\operatorname{ker}^{0}$ denotes the connected component of the identity of the kernel. In order to prove (7) we consider the normalization $\pi: \amalg Y_{i} \rightarrow Y$ and the following commutative diagram

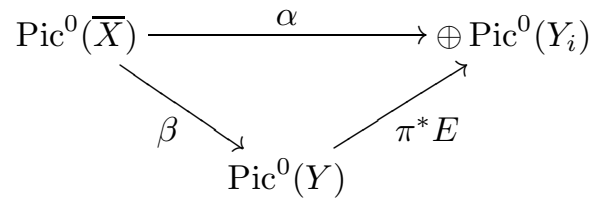

Now, because of [26, Exposé XII, Prop.2.3] (cf. [10]) the morphism

$$
\pi^{*}: \operatorname{Pic}(Y) \rightarrow \oplus \operatorname{Pic}\left(Y_{i}\right)
$$

is representable by an affine morphism. Then $\beta\left(\operatorname{ker}^{0} \alpha\right)=0$, since $\operatorname{ker}^{0} \alpha$ is an abelian variety. Since we obviously have $\operatorname{ker} \beta \subset \operatorname{ker} \alpha$, we must have $\operatorname{ker}^{0} \alpha=\operatorname{ker}^{0} \beta$ which is the claimed equality (7). 


\subsection{Definition of $\mathrm{Pic}^{-}$}

Let $X$ be an equidimensional variety over $k=\bar{k}$ of characteristic 0 . As before, let $\bar{X}$ be a good, normal crossing compactification of a resolution $f: \widetilde{X} \rightarrow X$ of $X$, with boundary divisor $Y$. Let $D$ be any Weil (or equivalently Cartier) divisor on $\bar{X}$ such that $\operatorname{supp}(D) \cap Y=\varnothing$, i.e., $D \in \operatorname{Div}(\bar{X}, Y)$; then $\left(\mathcal{O}_{\bar{X}}(D), 1\right)$ defines an element $[D] \in \operatorname{Pic}(\bar{X}, Y)$, where 1 denotes the tautological section of $\mathcal{O}_{\bar{X}}(D)$, trivializing it on $\bar{X}-D$, and hence also on $Y$.

We say that a divisor $D \in \operatorname{Div}(\bar{X}, Y)$ is algebraically equivalent to zero relative to $Y$ if $[D] \in \operatorname{Pic}^{0}(\bar{X}, Y)$ and we denote by $\operatorname{Div}^{0}(\bar{X}, Y) \subset \operatorname{Div}(\bar{X}, Y)$ the subgroup of divisors algebraically equivalent to zero relative to $Y$.

Let $\bar{S}$ be the closure of $\widetilde{S}$ in $\bar{X}$; then $\bar{S} \cup Y$ has normal crossings as well, since $\bar{X}$ is "good". Recall that $\operatorname{Div}{ }_{S}(\bar{X}, Y) \subset \operatorname{Div}(\bar{X}, Y)$ denotes the group of divisors $D$ on $\bar{X}$ supported on $\bar{S}$ such that $\operatorname{supp}(D) \cap Y=\varnothing$, i.e., it is the free abelian group on the compact irreducible components of $\widetilde{S}$. We have a push-forward on Weil divisors $f_{*}: \operatorname{Div}_{\widetilde{S}}(\widetilde{X}) \rightarrow \operatorname{Div}_{S}(X)$ and we let $\operatorname{Div}_{\widetilde{S} / S}(\widetilde{X})$ be the kernel of $f_{*}$.

We finally denote by $\operatorname{Div} \frac{0}{S} / S(\bar{X}, Y)$ the intersection of $\operatorname{Div}_{\widetilde{S} / S}(\widetilde{X})$ with $\operatorname{Div} \frac{0}{S}(\bar{X}, Y)$. Thus $\operatorname{Div} \frac{0}{S / S}(\bar{X}, Y)$ is the group of divisors on $\bar{X}$ which are linear combinations of compact divisorial components in $\widetilde{S}$, which have trivial push-forward under $f$ and which are algebraically equivalent to zero relative to $Y$.

Definition 2.2.1. - Let $X$ be an equidimensional variety over $k=\bar{k}$. With the hypothesis and notation as above we define the following 1-motive

$$
\operatorname{Pic}^{-}(X) \stackrel{\text { def }}{=}\left[\operatorname{Div} \frac{0}{S / S}(\bar{X}, Y) \stackrel{u}{\longrightarrow} \operatorname{Pic}^{0}(\bar{X}, Y)\right]
$$

where $u(D)=[D]$. We call $\mathrm{Pic}^{-}(X)$ the homological Picard 1-motive of $X$.

For any closed subscheme $Z \subset \bar{X}$ we define the following 1-motive

$$
\operatorname{Pic}^{+}(\bar{X}-Z, Y) \stackrel{\text { def }}{=}\left[\operatorname{Div}_{Z}^{0}(\bar{X}, Y) \stackrel{u}{\longrightarrow} \operatorname{Pic}^{0}(\bar{X}, Y)\right] .
$$

If $Z$ is the union of all compact components of divisors in $\widetilde{S}$, we then remark that $\mathrm{Pic}^{-}(X)$ is a sub-1-motive of $\mathrm{Pic}^{+}(\bar{X}-Z, Y)$.

If $X$ is an arbitrary $n$-dimensional variety over $k=\bar{k}$, let $X^{(n)}$ denote the union of its $n$-dimensional irreducible components. Define

$$
\operatorname{Pic}^{-}(X) \stackrel{\text { def }}{=} \operatorname{Pic}^{-}\left(X^{(n)}\right) \text {. }
$$

We next show that our definition of $\mathrm{Pic}^{-}(X)$ is independent of the choices made, i.e., of the resolution $\tilde{X}$ and compactification $\bar{X}$ as above, when $X$ is equidimensional (cf. also Remark 2.5.5). 


\subsection{Independence of resolutions and compactifications}

For an equidimensional $k$-variety $X$ as above, consider two resolutions of singularities $f^{\prime}: X^{\prime} \rightarrow X$ and $f^{\prime \prime}: X^{\prime \prime} \rightarrow X$ of $X$, with corresponding good compactifications $\bar{X}^{\prime}$ and $\bar{X}^{\prime \prime}$. We then can find a third resolution $f: \tilde{X} \rightarrow X$ dominating both $X^{\prime}$ and $X^{\prime \prime}$, and choose a compactification $\bar{X}$ which is a resolution of the closure of (the isomorphic image of) $X_{\text {reg }}=X-S$ in $\bar{X}^{\prime} \times \bar{X}^{\prime \prime}$, which is also a good normal crossing compactification of $\widetilde{X}$.

Hence, to prove that $\mathrm{Pic}^{-}(X)$ is independent of the choices made, it suffices to consider the following situation. Let $f_{1}: \widetilde{X}_{1} \rightarrow X$ be a resolution with good normal crossing compactification $\bar{X}_{1}$, and let $f_{2}: \widetilde{X}_{2} \rightarrow X$ be another one, with good normal crossing compactification $\bar{X}_{2}$, such that we have a morphism $f: \bar{X}_{2} \rightarrow \bar{X}_{1}$ whose restriction $f: \widetilde{X}_{2} \rightarrow \widetilde{X}_{1}$ is a proper morphism of $X$-schemes, necessarily a birational morphism. Under these conditions, we wish to show that $\mathrm{Pic}^{-}(X)$ defined using either $\bar{X}_{1}$ or $\bar{X}_{2}$ coincide.

Let $Y_{i}=\bar{X}_{i}-\widetilde{X}_{i}$ for $i=1,2$. We then clearly have a morphism of 1-motives

$$
\left[\operatorname{Div} \frac{0}{S_{1 / S}}\left(\bar{X}_{1}, Y_{1}\right) \rightarrow \operatorname{Pic}^{0}\left(\bar{X}_{1}, Y_{1}\right)\right] \rightarrow\left[\operatorname{Div} \frac{0}{S_{2} / S}\left(\bar{X}_{2}, Y_{2}\right) \rightarrow \operatorname{Pic}^{0}\left(\bar{X}_{2}, Y_{2}\right)\right]
$$

given by pulling back cycles and line bundles. It suffices to prove this is an isomorphism of 1-motives.

We first claim that there is an isomorphism of semi-abelian varieties

$$
\operatorname{Pic}^{0}\left(\bar{X}_{1}, Y_{1}\right) \cong \operatorname{Pic}^{0}\left(\bar{X}_{2}, Y_{2}\right) \text {. }
$$

In fact we have the following diagram

$$
\begin{array}{ccc}
\operatorname{Pic}^{0}\left(\bar{X}_{1}\right) & \cong \operatorname{Pic}^{0}\left(\bar{X}_{2}\right) \\
\downarrow & & \downarrow \\
\operatorname{Pic}\left(Y_{1}\right) & \hookrightarrow & \operatorname{Pic}\left(Y_{2}\right)
\end{array}
$$

where the bottom arrow is injective since $f_{*}\left(\mathcal{O}_{Y_{2}}\right)=\mathcal{O}_{Y_{1}}$ (because $Y_{1}$ is semi-normal, and $Y_{2} \rightarrow Y_{1}$ has connected fibers). Thus the kernels of the restrictions are the same, and so, regarding the relative $\mathrm{Pic}^{0}$ as an extension (by (5)) and using Proposition 2.1.2, the claim is clear.

Now we have a splitting of the pull-back map

$$
\left.\operatorname{Div}{\frac{0}{S_{1} / S}}_{\left(\bar{X}_{1}\right.}, Y_{1}\right) \stackrel{f^{*}}{\longrightarrow} \operatorname{Div} \frac{0}{S}_{2 / S}\left(\bar{X}_{2}, Y_{2}\right)
$$

using proper push-forward $f_{*}$ of divisors; we thus have

$$
\operatorname{Div} \frac{0}{S_{1} / S}\left(\bar{X}_{1}, Y_{1}\right) \oplus G \cong \operatorname{Div} \frac{0}{S_{2} / S}\left(\bar{X}_{2}, Y_{2}\right)
$$

where $G \stackrel{\text { def }}{=}\left\{D \in \operatorname{Div} \frac{0}{S_{2} / S}\left(\bar{X}_{2}, Y_{2}\right) \mid f_{*}(D)=0\right\}$. We will show that $G=0$. Since $\bar{X}_{2}$ and $\bar{X}_{1}$ are birational,

$$
f_{*}: \operatorname{Pic}^{0}\left(\bar{X}_{2}\right) \stackrel{\cong}{\longrightarrow} \operatorname{Pic}^{0}\left(\bar{X}_{1}\right)
$$


we note that if $D \in G$, then $D$ is linearly equivalent to zero on $\bar{X}_{2}$. Thus $D=\operatorname{div}(r)_{\bar{X}_{2}}$ where $r$ is a rational function on $\bar{X}_{2}$, and therefore also on $\bar{X}_{1}$. But

$$
\operatorname{div}(r)_{\bar{X}_{1}}=f_{*}(D)=0,
$$

whence $r$ is constant.

REMARK 2.3.1. - We remark that in our definition of $\mathrm{Pic}^{-}$, we can allow $f: \widetilde{X} \rightarrow X$ to be a birational proper morphism from a smooth variety, which is not necessarily a resolution of singularities of $X$. In fact, for any birational proper morphism $g$ : $\widetilde{X}^{\prime} \rightarrow \widetilde{X}$ between two such smooth $X$-varieties we can choose compactifications such that $g$ induces a morphism $\bar{g}: \bar{X}^{\prime} \rightarrow \bar{X}$. By arguing as above we then see that $\operatorname{Pic}^{0}(\bar{X}, Y) \cong \operatorname{Pic}^{0}\left(\bar{X}^{\prime}, Y^{\prime}\right)$ and $\operatorname{Div} \frac{0}{S} / S(\bar{X}, Y) \cong \operatorname{Div}_{S^{\prime} / S}^{0}\left(\bar{X}^{\prime}, Y^{\prime}\right)$.

\subsection{Hodge realization of $\mathrm{Pic}^{-}$}

In order to deal with the Hodge realization of $\mathrm{Pic}^{-}$the following results are needed.

Lemma 2.4.1. - Let $\bar{X}$ and $Y$ be as above, with $k=\mathbb{C}$. We then have the following properties of (the group of $\mathbb{C}$-points of) $\operatorname{Pic}(\bar{X}, Y)$.

a) There is an exact sequence

$$
H^{1}(\bar{X}, Y ; \mathbb{Z}(1)) \rightarrow H^{1}\left(\bar{X}, \mathcal{O}_{\bar{X}}(-Y)\right) \rightarrow \operatorname{Pic}(\bar{X}, Y) \stackrel{c l}{\longrightarrow} H^{2}(\bar{X}, Y ; \mathbb{Z}(1)) .
$$

b) There is an isomorphism

$$
\operatorname{Pic}^{0}(\bar{X}, Y) \cong \operatorname{ker}\left(\operatorname{Pic}(\bar{X}, Y) \stackrel{c \ell}{\longrightarrow} H^{2}(\bar{X}, Y ; \mathbb{Z}(1))\right) .
$$

c) There is an isomorphism

$$
J^{1}(\bar{X}, Y) \stackrel{\text { def }}{=} \frac{H^{1}(\bar{X}, Y ; \mathbb{C}(1))}{F^{0}+H^{1}(\bar{X}, Y ; \mathbb{Z}(1))} \cong \frac{H^{1}\left(\bar{X}, \mathcal{O}_{\bar{X}}(-Y)\right)}{H^{1}(\bar{X}, Y ; \mathbb{Z}(1))} .
$$

d) Under the isomorphism (induced by a)-c))

$$
\operatorname{Pic}^{0}(\bar{X}, Y) \cong J^{1}(\bar{X}, Y)
$$

the mapping $D \longmapsto[D]=\left(\mathcal{O}_{\bar{X}}(D), 1\right)$ from $\operatorname{Div}^{0}(\bar{X}, Y)$ to $\operatorname{Pic}^{0}(\bar{X}, Y)$ is identified with the extension class map, for the mixed Hodge structure, determined by the support of $D$.

e) Let $Z$ be a closed subscheme $Z \subset \bar{X}$ such that $Z \cap Y=\varnothing$. Then

$$
T_{\text {Hodge }}\left(\operatorname{Pic}^{+}(\bar{X}-Z, Y)\right) \cong H^{1}(\bar{X}-Z, Y ; \mathbb{Z}(1)) .
$$

Proof. - We first claim that

$$
\frac{H^{1}(\bar{X}, Y ; \mathbb{C}(1))}{F^{0}} \cong H^{1}\left(\bar{X}, \mathcal{O}_{\bar{X}}(-Y)\right) .
$$

To see this, we consider the twisted $\log$ De Rham complex $\Omega_{\bar{X}}(\log (Y))(-Y)$. It is well-known (see [51], page 4 for a quick proof) that its hypercohomology groups 
are the relative cohomology groups $H^{*}(\bar{X}, Y ; \mathbb{C})$, the Hodge-De Rham filtration on

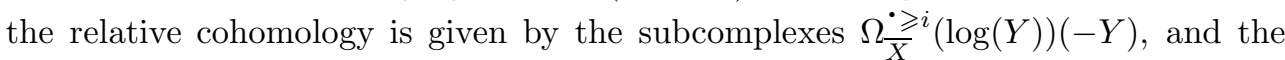
corresponding hypercohomology spectral sequence degenerates at $E_{1}$. We then have

$$
\frac{H^{k}(\bar{X}, Y, \mathbb{C})}{F^{i}} \cong \mathbb{H}^{k}\left(\bar{X}, \Omega_{\bar{X}}^{\cdot<i}(\log (Y))(-Y)\right) .
$$

The claimed isomorphism is obtained from (9) for $k=i=1$.

Let $\mathcal{O}_{\left(\bar{X}_{\text {an }}, Y_{\text {an }}\right)}^{*}$ be the sheaf on $\bar{X}_{\text {an }}$ given by the kernel of $\mathcal{O}_{\bar{X}_{\mathrm{an}}}^{*} \rightarrow i_{*} \mathcal{O}_{Y_{\mathrm{an}}}^{*}$ where $i: Y \hookrightarrow \bar{X}$ is the inclusion. We have

$$
\operatorname{Pic}(\bar{X}, Y) \cong H^{1}\left(\bar{X}_{\mathrm{an}}, \mathcal{O}_{\left(\bar{X}_{\mathrm{an}}, Y_{\mathrm{an}}\right)}^{*}\right)
$$

because of (5) and GAGA. We have that $\mathcal{O}_{\bar{X}_{\text {an }}}\left(-Y_{\text {an }}\right)$ is the kernel of the canonical map $\mathcal{O}_{\bar{X}_{\text {an }}} \rightarrow i_{*} \mathcal{O}_{Y_{\text {an }}}$; therefore, by the exponential sequences on $\bar{X}_{\text {an }}$ and $Y_{\text {an }}$, since $i_{*}$ is an exact functor, we get the following induced relative exponential exact sequence of sheaves on $\bar{X}_{\text {an }}$

$$
0 \rightarrow j_{!}(\mathbb{Z}(1)) \rightarrow \mathcal{O}_{\bar{X}_{\mathrm{an}}}\left(-Y_{\mathrm{an}}\right) \rightarrow \mathcal{O}_{\left(\bar{X}_{\mathrm{an}}, Y_{\mathrm{an}}\right)}^{*} \rightarrow 0
$$

where $j$ ! is the extension by zero functor along $j: \bar{X}_{\text {an }}-Y_{\text {an }} \hookrightarrow \bar{X}_{\text {an }}$.

We then get the following exact sequence of cohomology groups

$$
\cdots \rightarrow H^{1}(\bar{X}, Y ; \mathbb{Z}(1)) \rightarrow H^{1}\left(\bar{X}, \mathcal{O}_{\bar{X}}(-Y)\right) \rightarrow \operatorname{Pic}(\bar{X}, Y) \stackrel{c \ell}{\longrightarrow} H^{2}(\bar{X}, Y ; \mathbb{Z}(1)) \rightarrow \cdots
$$

The exact sequence in $a)$ is then obtained. Since $H^{2}(\bar{X}, Y ; \mathbb{Z}(1))$ is finitely generated and ker $c \ell$ is divisible, we get $b$ ). From (9) we then get $c$ ).

Part $d)$ is well known if $Y=\varnothing(e . g$. , see [12]). In order to show part $d)$ in general, we can proceed as follows. By considering relative Deligne-Beilinson cohomology $H_{\mathcal{D}}^{*}(\bar{X}, Y ; \mathbb{Z}(*))$ we get a canonical cycle class map

$$
c_{1}: \operatorname{Pic}(\bar{X}, Y) \rightarrow H_{\mathcal{D}}^{2}(\bar{X}, Y ; \mathbb{Z}(1))
$$

Moreover, $c_{1}$ is an isomorphism, fitting into the following commutative diagram with exact rows

$$
\begin{array}{ccccc}
\operatorname{Pic}^{0}(\bar{X}, Y) & \operatorname{Pic}(\bar{X}, Y) & \stackrel{c \ell}{\longrightarrow} H^{2}(\bar{X}, Y ; \mathbb{Z}(1)) \rightarrow & H^{2}\left(\bar{X}, \mathcal{O}_{\bar{X}}(-Y)\right) \\
\quad \cong \downarrow & \cong \downarrow c_{1} & & \| \downarrow \\
J^{1}(\bar{X}, Y) & \hookrightarrow H_{\mathcal{D}}^{2}(\bar{X}, Y ; \mathbb{Z}(1)) & \rightarrow & H^{2}(\bar{X}, Y ; \mathbb{Z}(1)) \rightarrow H^{2}(\bar{X}, Y ; \mathbb{C}(1)) / F^{0}
\end{array}
$$

obtained from (9) and (11). For any closed subscheme $Z \subset \bar{X}$ with $Z \cap Y=\varnothing$, we then have the following commutative diagram of cohomology groups having exact 
rows and columns

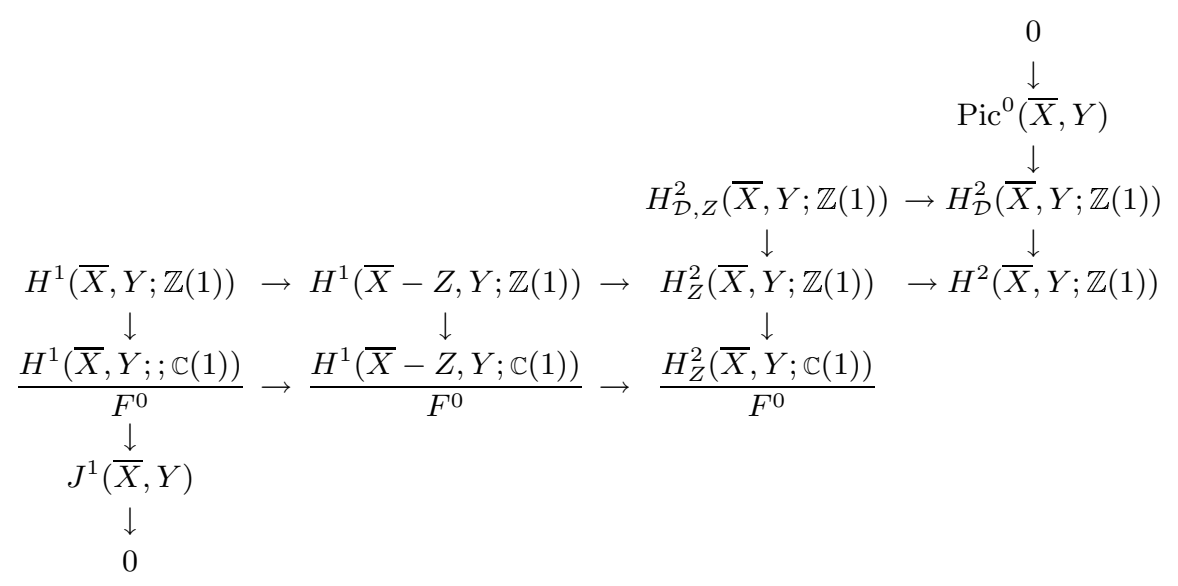

Here $H_{\mathcal{D}, Z}^{*}(\bar{X}, Y ; \mathbb{Z}(\cdot)) \cong H_{\mathcal{D}, Z}^{*}(\bar{X} ; \mathbb{Z}(\cdot))$ is the (relative) Deligne-Beilinson cohomology of $(\bar{X}, Y)$ with support in $Z$.

Let $Z$ be the support of a divisor $D \in \operatorname{Div}(\bar{X}, Y)$, i.e., $Z \cap Y=\varnothing$. We then have that

$$
H_{Z}^{*}(\bar{X}, Y ; \mathbb{Z}(1)) \cong H_{Z}^{*}(\bar{X}, \mathbb{Z}(1)) .
$$

In particular: $H_{Z}^{1}(\bar{X}, Y ; \mathbb{Z}(1))=0$ and $H_{Z}^{2}(\bar{X}, Y ; \mathbb{Z}(1))$ is purely of type $(0,0)$; in fact, we have an isomorphism

$$
H_{\mathcal{D}, Z}^{2}(\bar{X}, Y ; \mathbb{Z}(1)) \cong H_{Z}^{2}(\bar{X}, Y ; \mathbb{Z}(1)) \cong \operatorname{Div}_{Z}(\bar{X}, Y) .
$$

The claim $d$ ) then follows from a diagram chase in the diagram above, using a general homological lemma [9, Lemma 2.8].

Part $e$ ) then follows from the diagram as well, yielding the following isomorphism, in the category of 1 -motives over $\mathbb{C}$,

$$
\begin{gathered}
\operatorname{Div}_{Z}^{0}(\bar{X}, Y) \stackrel{e}{\longrightarrow} J^{1}(\bar{X}, Y) \\
\| \\
\operatorname{Div}_{Z}^{0}(\bar{X}, Y) \stackrel{u}{\longrightarrow} \operatorname{Pic}^{0}(\bar{X}, Y)
\end{gathered}
$$

where $e$ denotes the extension class map determined by $H^{1}(\bar{X}-Z, Y ; \mathbb{Z}(1))$, regarded as an extension of mixed Hodge structures.

For the following duality result we refer to the book of Spanier [50], giving a proof in the topological setting. In order to deduce such a duality statement for different cohomology theories, as well as compatibilities between them, we are going to give a proof in the Grothendieck-Verdier duality style.

Lemma 2.4.2. - Let $M$ be a compact smooth $n$-dimensional $\mathbb{C}$-variety. Let $A+B$ be a reduced normal crossing divisor in $M$ such that $A \cap B=\varnothing$. Then there is a 
duality isomorphism

$$
H^{r}(M-A, B ; \mathbb{Z}) \cong H_{2 n-r}(M-B, A ; \mathbb{Z}(-n))
$$

in the category of mixed Hodge structures. Moreover i) this isomorphism is functorial, i.e., if $A^{\prime} \subset A, B \subset B^{\prime}$ and $A^{\prime}+B^{\prime}$ is also a normal crossing divisor such that $A^{\prime} \cap B^{\prime}=\varnothing$ then the following diagram

$$
\begin{array}{ccc}
H^{r}\left(M-A^{\prime}, B^{\prime} ; \mathbb{Z}\right) & \rightarrow & H^{r}(M-A, B ; \mathbb{Z}) \\
\cong \downarrow & \downarrow \cong \\
H_{2 n-r}\left(M-B^{\prime}, A^{\prime} ; \mathbb{Z}(-n)\right) & \rightarrow H_{2 n-r}(M-B, A ; \mathbb{Z}(-n))
\end{array}
$$

commutes, in the category of mixed Hodge structures; finally ii) this duality isomorphism is compatible with the Poincaré-Lefschetz duality, i.e., if $B=B^{\prime}$ and $A^{\prime} \subset A$ as above then the following diagram, whose rows are parts of long exact sequences, commutes

$$
\begin{aligned}
& H^{r}\left(M-A^{\prime}, B ; \mathbb{Z}\right) \quad \rightarrow \quad H^{r}(M-A, B ; \mathbb{Z}) \quad \rightarrow \quad H^{r+1}\left(M-A^{\prime}, M-A ; \mathbb{Z}\right) \\
& \cong \downarrow \quad \cong \downarrow \quad \cong \downarrow \\
& H_{2 n-r}\left(M-B, A^{\prime} ; \mathbb{Z}(-n)\right) \rightarrow H_{2 n-r}(M-B, A ; \mathbb{Z}(-n)) \rightarrow H_{2 n-r-1}\left(A, A^{\prime} ; \mathbb{Z}(-n)\right)
\end{aligned}
$$

in the category of mixed Hodge structures.

Proof. - Let $V=M-(A \cup B), V_{A}=M-A$ and $V_{B}=M-B$ be the corresponding open subsets; we have a diagram

$$
\begin{array}{crr}
V & \stackrel{\beta}{\rightarrow} V_{B} \\
\alpha \downarrow & \downarrow \gamma \\
V_{A} \stackrel{\delta}{\rightarrow} & M
\end{array}
$$

We let ${ }^{A} i: A \hookrightarrow V_{B},{ }^{B} i: B \hookrightarrow V_{A}$ denote the closed imbeddings. Let $\pi: M \rightarrow k$ be the structure morphism. Because of the canonical exact sequence

$$
0 \rightarrow \alpha ! \mathbb{Z}_{V} \rightarrow \mathbb{Z}_{V_{A}} \rightarrow{ }^{B} i_{*} \mathbb{Z}_{B} \rightarrow 0
$$

of sheaves on $V_{A}$ we have

$$
\begin{aligned}
H^{r}\left(V_{A}, B\right) & \cong \operatorname{Hom}\left(\mathbb{Z}_{V_{A}}, \alpha ! \mathbb{Z}_{V}[r]\right) \\
& \cong \operatorname{Hom}\left(\mathbb{Z}_{M}, \delta_{*} \alpha ! \mathbb{Z}_{V}[r]\right)
\end{aligned}
$$

where the Hom is taken in the derived category. Thus

$$
\operatorname{RHom}\left(\mathbb{Z}_{M}, \delta_{*} \alpha ! \mathbb{Z}_{V}\right) \cong \mathrm{R} \Gamma\left(M, \delta_{*} \alpha ! \mathbb{Z}_{V}\right)
$$

computes the singular cohomology of the pair $(M-A, B)$. Now we have

$$
H_{2 n-r}(M-B, A) /(\text { torsion }) \cong \operatorname{Hom}\left(H^{2 n-r}(M-B, A), \mathbb{Z}\right)
$$


as mixed Hodge structures. Similarly the complex $\mathrm{R} \Gamma\left(M, \gamma_{*} \beta_{!} \mathbb{Z}_{V}\right)$ computes the cohomology of the pair $(M-B, A)$ and we have

$$
\begin{aligned}
\operatorname{RHom}\left(\mathrm{R} \Gamma\left(M, \gamma_{*} \beta_{!} \mathbb{Z}_{V}\right)[2 n], \mathbb{Z}(-n)\right) & \cong \operatorname{RHom}\left(\mathrm{R} \pi_{!}\left(\gamma_{*} \beta_{!} \mathbb{Z}_{V}\right)[2 n], \mathbb{Z}(-n)\right) \\
& \cong \operatorname{RHom}\left(\gamma_{*} \beta_{!} \mathbb{Z}_{V}, \pi^{!} \mathbb{Z}[-2 n](-n)\right) \\
& \cong \operatorname{RHom}\left(\delta_{!} \alpha_{*} \mathbb{Z}_{V}, \mathbb{Z}_{M}\right) \\
& \cong \operatorname{R\Gamma }\left(M, \mathcal{R} H o m\left(\delta_{!} \alpha_{*} \mathbb{Z}_{V}, \mathbb{Z}_{M}\right)\right)
\end{aligned}
$$

by using Grothendieck-Verdier duality, i.e., $\mathrm{R} \pi$ ! is left adjoint to $\pi^{\text {! }}$, where the dualizing complex $\omega_{M} \stackrel{\text { def }}{=} \pi^{!} \mathbb{Z}$ is given by $\pi^{!} \mathbb{Z} \cong \mathbb{Z}_{M}[2 n](n)$, and the obvious equality $\gamma_{*} \beta_{!}=\delta_{!} \alpha_{*}$. Now we can argue that

$$
\begin{aligned}
\mathcal{R H o m}\left(\delta_{!} \alpha_{*} \mathbb{Z}_{V}, \mathbb{Z}_{M}\right) & \cong \delta_{*} \mathcal{R H o m}\left(\alpha_{*} \mathbb{Z}_{V}, \delta^{!} \mathbb{Z}_{M}\right) \\
& \cong \delta_{*} \mathcal{R} \operatorname{Hom}\left(\alpha_{*} \mathbb{Z}_{V}, \mathbb{Z}_{V_{A}}\right) \\
& \cong \delta_{*} \alpha ! \mathbb{Z}_{V}
\end{aligned}
$$

where the last equality is given by the following isomorphism

$$
\alpha ! \mathbb{Z}_{V} \stackrel{\cong}{\longrightarrow} \operatorname{Hom}\left(\alpha_{*} \mathbb{Z}_{V}, \mathbb{Z}_{V_{A}}\right)
$$

The isomorphism (14) can be obtained from biduality for constructible sheaves. In fact, let $\omega_{V_{A}}$ be the dualizing sheaf; since $V_{A}$ is smooth $\omega_{V_{A}}[-2 n](-n) \cong \mathbb{Z}_{V_{A}}$, therefore, by biduality, the formula (14) is equivalent to

$$
\mathcal{R H o m}\left(\alpha ! \mathbb{Z}_{V}, \mathbb{Z}_{V_{A}}\right) \cong \alpha_{*} \mathbb{Z}_{V}
$$

which is clear since

$$
\begin{aligned}
\mathcal{R H o m}\left(\alpha ! \mathbb{Z}_{V}, \omega_{V_{A}}[-2 n](-n)\right) & \cong \alpha_{*} \mathcal{R H o m}\left(\mathbb{Z}_{V}, \alpha^{!} \omega_{V_{A}}[-2 n](-n)\right) \\
& \cong \alpha_{*} \mathcal{R H o m}\left(\mathbb{Z}_{V}, \omega_{V}[-2 n](-n)\right) \\
& \cong \alpha_{*} \omega_{V}[-2 n](-n) \\
& \cong \alpha_{*} \mathbb{Z}_{V}
\end{aligned}
$$

where we have used that $\alpha^{!} \omega_{V_{A}}$ is the dualizing sheaf on $V$.

Summarizing, we have obtained the following isomorphism

$$
\operatorname{RHom}\left(\mathrm{R} \Gamma\left(M, \gamma_{*} \beta_{!} \mathbb{Z}_{V}\right)[2 n], \mathbb{Z}(-n)\right) \cong \mathrm{R} \Gamma\left(M, \delta_{*} \alpha ! \mathbb{Z}_{V}\right)
$$

yielding the claimed duality isomorphism of groups.

In order to show the compatibility of the above with the mixed Hodge structures we consider the following induced pairing in the derived category

$$
\gamma_{*} \beta_{!} \mathbb{Z}_{V}[2 n](n) \stackrel{L}{\otimes} \delta_{*} \alpha ! \mathbb{Z}_{V} \rightarrow \pi^{!} \mathbb{Z}
$$


This pairing is unique (up to a unique integer multiple); indeed, we have

$$
\begin{aligned}
\operatorname{Hom}\left(\gamma_{*} \beta_{!} \mathbb{Z}_{V}\right. & {\left.[2 n](n) \stackrel{L}{\otimes} \delta_{*} \alpha_{!} \mathbb{Z}_{V}, \pi^{!} \mathbb{Z}\right) } \\
& \cong \operatorname{Hom}\left(\gamma_{*} \beta_{!} \mathbb{Z}_{V}[2 n](n) \stackrel{L}{\mathcal{R}} \operatorname{Hom}\left(\delta_{!} \alpha_{*} \mathbb{Z}_{V}[2 n](n), \pi^{!} \mathbb{Z}\right), \pi^{!} \mathbb{Z}\right) \\
& \cong \operatorname{Hom}\left(\gamma_{*} \beta_{!} \mathbb{Z}_{V}[2 n](n), \mathcal{R} \operatorname{Hom}\left(\mathcal{R} H o m\left(\delta_{!} \alpha_{*} \mathbb{Z}_{V}[2 n](n), \pi^{!} \mathbb{Z}\right), \pi^{!} \mathbb{Z}\right)\right) \\
& \cong \operatorname{Hom}\left(\gamma_{*} \beta_{!} \mathbb{Z}_{V}[2 n](n), \delta_{!} \alpha_{*} \mathbb{Z}_{V}[2 n](n)\right) \\
& \cong \operatorname{Hom}\left(\delta_{!} \alpha_{*} \mathbb{Z}_{V}[2 n](n), \delta_{!} \alpha_{*} \mathbb{Z}_{V}[2 n](n)\right) \\
& \cong \operatorname{Hom}\left(\mathbb{Z}_{V}(n), \mathbb{Z}_{V}(n)\right) \\
& \cong \mathbb{Z}
\end{aligned}
$$

where we have used the formula (14), biduality for the constructible sheaf $\delta_{!} \alpha_{*} \mathbb{Z}_{V}$ and the standard formalism of derived categories. The same arguments apply to the constant sheaves $\mathbb{Q}$ or $\mathbb{C}$.

By Saito's theory of mixed Hodge modules [44], [45], all of the above constructions and isomorphisms can (after $\otimes \mathbb{Q}$ ) be "lifted" in a natural way to the derived category of mixed Hodge modules. In particular, we see that our duality isomorphism is compatible with the mixed Hodge structures as claimed.

We leave to the reader the analogous proofs of the assertions about functoriality, and compatibility with Poincaré-Lefschetz duality.

REMARK 2.4.3. - We remark that, for the truth of the Lemma 2.4.2 the assumption that $A \cap B=\varnothing$ is not really needed: it suffices to assume that $A+B$ is a reduced normal crossing divisor on $M$, but the proof in this case is a bit more involved.

REMARK 2.4.4. - Let $\Omega_{M}^{\cdot}(\log (N))(-D)$ be the $\log$ De Rham complex with terms

$$
\Omega_{M}^{i}(\log (N)) \otimes \mathcal{O}_{M} \mathcal{O}_{M}(-D)
$$

for $D$ any Weil divisor on $M$ and $N$ a reduced normal crossing divisor in $M$ which contains $\operatorname{supp}(D)$. Let $j: M-N \hookrightarrow M$ be the inclusion; we then have a quasiisomorphism

$$
j_{!} \mathbb{C} \stackrel{\cong}{\longrightarrow} \Omega_{M}^{\cdot}(\log (N))(-N) .
$$

In the notation of Lemma 2.4.2 we remark that the following pairing

$$
\begin{gathered}
\Omega_{M}^{\cdot}(\log (A+B))(-A) \otimes_{\mathbb{C}} \Omega_{M}^{\cdot}(\log (A+B))(-B) \\
\downarrow \\
\Omega_{M}^{\cdot}(\log (A+B))(-A-B) \\
\downarrow \\
\Omega_{M}^{\cdot}
\end{gathered}
$$

can be identified with the sheaf theoretic pairing given by (15) (up to a unique scalar)

$$
\gamma_{*} \beta_{!} \mathbb{C}_{V} \stackrel{L}{\otimes} \delta_{*} \alpha_{!} \mathbb{C}_{V} \longrightarrow j_{!} \mathbb{C}_{V} \longrightarrow \mathbb{C}_{M}
$$


This is valid in the filtered derived category since ( $c f .[51])$ we obtain the Hodge-De Rham filtrations by truncation of the above log De Rham complexes; this gives a "direct" proof that the duality isomorphism in the Lemma 2.4.2 is compatible, after $\otimes \mathbb{C}$, with the respective Hodge filtrations.

As remarked by Deligne [17], the duality isomorphism is, after $\otimes \mathbb{Q}$, also compatible with weight filtrations, because this is true $\otimes \mathbb{Q}_{\ell}$, by comparison with étale cohomology, and the Weil conjectures.

For another related compatibility argument using mixed Hodge complexes we refer to $[51]$.

We then have the following key result.

Theorem 2.4.5. - Let $X$ be a $\mathbb{C}$-variety of dimension $n$. Then

$$
T_{\text {Hodge }}\left(\operatorname{Pic}^{-}(X)\right) \cong H_{2 n-1}(X, \mathbb{Z}(1-n)) \text {. }
$$

Proof. - We first make a reduction to the case when $X$ is equidimensional. Let $X^{(n)} \subset X$ be the union of the $n$-dimensional irreducible components of $X$. Then by definition, $\mathrm{Pic}^{-}(X)=\mathrm{Pic}^{-}\left(X^{(n)}\right)$. On the other hand, the natural map

$$
H_{2 n-1}\left(X^{(n)}, \mathbb{Z}(1-n)\right) \rightarrow H_{2 n-1}(X, \mathbb{Z}(1-n))
$$

is an isomorphism of mixed Hodge structures.

Now for equidimensional $X$, let $f: \widetilde{X} \rightarrow X$ be a resolution, with a good normal crossing compactification $\bar{X}$ with boundary $Y$. As before, let $S$ be the singular locus of $X, \widetilde{S}=f^{-1}(S)$, and $\bar{S} \subset \bar{X}$ the Zariski closure of $\widetilde{S}$.

Associated to the cartesian square

$$
\begin{array}{lll}
\widetilde{S} & \hookrightarrow & \widetilde{X} \\
\downarrow & & \downarrow f f \\
S & \hookrightarrow & X
\end{array}
$$

there is a Mayer-Vietoris long exact sequence of mixed Hodge structures on singular homology yielding the following extension

$$
0 \rightarrow H_{2 n-1}(\tilde{X}, \mathbb{Z}(1-n)) \rightarrow H_{2 n-1}(X, \mathbb{Z}(1-n)) \rightarrow L_{X} \rightarrow 0
$$

where

$$
L_{X}=\operatorname{ker} H_{2 n-2}(\widetilde{S}, \mathbb{Z}(1-n)) \rightarrow H_{2 n-2}(\widetilde{X}, \mathbb{Z}(1-n)) \oplus H_{2 n-2}(S, \mathbb{Z}(1-n)) .
$$

Now we claim:

(i) $H_{2 n-2}(\widetilde{S}, \mathbb{Z}(1-n)) \cong \operatorname{Div} \bar{S}(\bar{X}, Y)$,

(ii) $f_{*}: H_{2 n-2}(\widetilde{S}, \mathbb{Z}(1-n)) \rightarrow H_{2 n-2}(S, \mathbb{Z}(1-n))$ is the proper push-forward of algebraic cycles, and

(iii) $H_{2 n-i}(\widetilde{X}, \mathbb{Z}(1-n)) \cong H^{i}(\bar{X}, Y)(1)$ as mixed Hodge structures. 
In fact $H_{2 n-2}(\widetilde{S}, \mathbb{Z}(1-n))$ is the free abelian group generated by the compact irreducible $(n-1)$-dimensional components of $\widetilde{S}$, and $H_{2 n-2}(S, \mathbb{Z}(1-n))$ has a similar description. Thus (i) - (ii) are clear and (iii) follows from Lemma 2.4.2 because $\widetilde{X}=\bar{X}-Y$. Moreover we have that the mapping

$$
H_{2 n-2}(\widetilde{S}, \mathbb{Z}(1-n)) \longrightarrow H_{2 n-2}(\widetilde{X}, \mathbb{Z}(1-n))
$$

induced by the inclusion $\widetilde{S} \hookrightarrow \widetilde{X}$, is just the cycle map relative to $Y$, i.e., the following diagram

commutes.

$$
\begin{array}{ccccc}
\operatorname{Div}_{\bar{S}}(\bar{X}, Y) & \stackrel{\cong}{\longrightarrow} & H_{2 n-2}(\widetilde{S}, \mathbb{Z}(1-n)) & \longrightarrow & H_{2 n-2}(\widetilde{X}, \mathbb{Z}(1-n)) \\
\downarrow & & \downarrow & & \\
\operatorname{Pic}(\bar{X}, Y) & = & \operatorname{Pic}(\bar{X}, Y) & \stackrel{c \ell}{\longrightarrow} & H^{2}(\bar{X}, Y)
\end{array}
$$

Since, by definition, the kernel of $c \ell$ is $\operatorname{Pic}^{0}(\bar{X}, Y)$ ( $c f$. Lemma 2.1.2), the lattice $L_{X}$ is canonically isomorphic to $\operatorname{Div} \frac{0}{S} / S(\bar{X}, Y)$. Moreover the exact sequence $(16)$ modulo torsion is canonically isomorphic to the following exact sequence

$$
0 \rightarrow H^{1}(\bar{X}, Y, \mathbb{Z}(1)) /(\text { torsion }) \rightarrow H_{2 n-1}(X, \mathbb{Z}(1-n)) /(\text { torsion }) \rightarrow L_{X} \rightarrow 0
$$

in the category of torsion free mixed Hodge structures. But $H^{1}(\bar{X}, Y)(1)$ is torsionfree, by the universal coefficient theorem in topology; hence so is $H_{2 n-1}(X, \mathbb{Z}(1-n))$. The Hodge structure on $L_{X}$ is pure of weight zero and type $(0,0)$; we then have

$$
W_{-i}\left(H_{2 n-1}(X, \mathbb{Z}(1-n))\right)=W_{-i}\left(H^{1}(\bar{X}, Y, \mathbb{Z}(1))\right), \quad i \geqslant 1 .
$$

We also have the following extension of mixed Hodge structures

$$
0 \rightarrow \frac{H^{0}(Y, \mathbb{Z})}{\operatorname{im} H^{0}(\bar{X}, \mathbb{Z})} \otimes \mathbb{Z}(1) \rightarrow H^{1}(\bar{X}, Y, \mathbb{Z}(1)) \rightarrow \operatorname{ker}\left(H^{1}(\bar{X}, \mathbb{Z}(1)) \rightarrow H^{1}(Y, \mathbb{Z}(1))\right) \rightarrow 0
$$

Thus the weight filtration of $H_{2 n-1}(X, \mathbb{Z}(1-n))$ admits the following description. Let

$$
r=\operatorname{rank} \frac{H^{0}(Y, \mathbb{Z})}{\operatorname{im} H^{0}(\bar{X}, \mathbb{Z})}
$$

then

$$
W_{-2}\left(H_{2 n-1}(X, \mathbb{Z}(1-n))\right) \cong \mathbb{Z}(1)^{\oplus r}
$$

and

$$
W_{-1}\left(H_{2 n-1}(X, \mathbb{Z}(1-n))\right) \cong H^{1}(\bar{X}, Y, \mathbb{Z}(1)) .
$$

Since $H^{1}(\bar{X}, \mathbb{Z}(1))$ is pure of weight -1 , we have

$$
\operatorname{ker}\left(H^{1}(\bar{X}, \mathbb{Z}(1)) \rightarrow H^{1}(Y, \mathbb{Z}(1))\right)=\operatorname{ker}\left(H^{1}(\bar{X}, \mathbb{Z}(1)) \rightarrow \oplus H^{1}\left(Y_{i}, \mathbb{Z}(1)\right)\right)
$$

whence

$$
\operatorname{gr}_{-1}^{W} H_{2 n-1}(X, \mathbb{Z}(1-n)) \cong \operatorname{ker}\left(H^{1}(\bar{X}, \mathbb{Z}(1)) \rightarrow \oplus H^{1}\left(Y_{i}, \mathbb{Z}(1)\right)\right)
$$

and

$$
\operatorname{gr}_{0}^{W} H_{2 n-1}(X, \mathbb{Z}(1-n)) \cong L_{X}
$$


Thus the 1 -motive associated (by Deligne) to $H_{2 n-1}(X, \mathbb{Z}(1-n))$ is given by the following

$$
\begin{gathered}
L_{X} \\
1 \rightarrow\left(\mathbb{C}^{*}\right)^{\oplus r} \rightarrow J^{1}\left(\frac{\downarrow^{e}}{X}, Y\right) \rightarrow \operatorname{ker}^{0}\left(J^{1}(\bar{X}) \rightarrow \oplus J^{1}\left(Y_{i}\right)\right) \rightarrow 0
\end{gathered}
$$

$\left(J^{1}(\bar{X}, Y)\right.$ was defined in Lemma $2.4 .1(\mathrm{c}) ; J^{1}(\bar{X}), J^{1}\left(Y_{i}\right)$ are similarly defined). Since

$$
T_{\text {Hodge }}\left(L_{X} \rightarrow J^{1}(\bar{X}, Y)\right) \cong H_{2 n-1}(X, \mathbb{Z}(1-n))
$$

by Deligne's construction, we are reduced to showing that

$$
\left[L_{X} \rightarrow J^{1}(\bar{X}, Y)\right] \cong \mathrm{Pic}^{-}(X)
$$

in the category of 1-motives over $\mathbb{C}$.

By Lemma 2.4.1 (cf. Proposition 2.1.2, where $T(\bar{X}, Y)(\mathbb{C})=\left(\mathbb{C}^{*}\right)^{\oplus r}$ and $A(\bar{X}, Y)$ is the above abelian variety) we have that

$$
\operatorname{Pic}^{0}(\bar{X}, Y) \cong J^{1}(\bar{X}, Y) .
$$

According to our definition of $\mathrm{Pic}^{-}(X)$ we are left to check that the following

$$
\begin{array}{cc}
L_{X} & \stackrel{e}{\longrightarrow} J^{1}(\bar{X}, Y) \\
\| & \downarrow \cong \\
\operatorname{Div} \frac{0}{S / S}(\bar{X}, Y) \stackrel{u}{\longrightarrow} \operatorname{Pic}^{0}(\bar{X}, Y)
\end{array}
$$

commutes. We will deduce this from Lemma 2.4.1(d)

The Mayer-Vietoris exact sequence yielding (16) is given by the following commutative diagram of mixed Hodge structures

$$
\begin{aligned}
& H_{2 n-1}(\widetilde{X}) \hookrightarrow H_{2 n-1}(\widetilde{X}, \widetilde{S}) \rightarrow H_{2 n-2}(\widetilde{S}) \rightarrow H_{2 n-2}(\widetilde{X}) \\
& \downarrow \quad \cong \downarrow \quad \downarrow \quad \downarrow \\
& H_{2 n-1}(X) \hookrightarrow H_{2 n-1}(X, S) \rightarrow H_{2 n-2}(S) \rightarrow H_{2 n-2}(X)
\end{aligned}
$$

which yields the following diagram of mixed Hodge structures

$$
\begin{aligned}
& 0 \rightarrow H_{2 n-1}(\tilde{X}, \mathbb{Z}(1-n)) \rightarrow H_{2 n-1}(X, \mathbb{Z}(1-n)) \rightarrow \operatorname{Div} \frac{0}{S} / S(\bar{X}, Y) \rightarrow 0 \\
& 0 \rightarrow H_{2 n-1}(\widetilde{X}, \mathbb{Z}(1-n)) \rightarrow \quad H_{2 n-1}(\widetilde{X}, \widetilde{S}) \quad \rightarrow \quad \operatorname{Div} \frac{0}{S}\left(\frac{\downarrow}{X}, Y\right) \rightarrow 0
\end{aligned}
$$

where

$$
\operatorname{ker}\left(H_{2 n-2}(\widetilde{S}) \rightarrow H_{2 n-2}(\tilde{X})\right) \cong \operatorname{Div} \frac{0}{S}(\bar{X}, Y) .
$$

Let $D \in L_{X}=\operatorname{Div} \frac{0}{S / S}(\bar{X}, Y)$; then $Z=\operatorname{supp}(D)$ is a closed subset of $\bar{X}$ such that $Z \cap Y=\varnothing$, and $D$ is homologically equivalent to zero relative to $Y$; we let

$$
\mathbb{Z}[Z]^{0} \stackrel{\text { def }}{=} \operatorname{ker}\left(\operatorname{Div}_{Z}^{0}(\bar{X}, Y) \rightarrow H^{2}(\bar{X}, Y ; \mathbb{Z}(1))\right) .
$$


We have the following diagram of torsion-free mixed Hodge structures

$$
\begin{aligned}
& 0 \rightarrow H^{1}(\bar{X}, Y ; \mathbb{Z}(1)) \quad \rightarrow \quad H^{1}(\bar{X}-Z, Y ; \mathbb{Z}(1)) \quad \rightarrow \quad \mathbb{Z}[Z]^{0} \rightarrow 0 \\
& 0 \stackrel{\cong}{\cong}(\widetilde{X}, \mathbb{Z}(1-n)) \rightarrow H_{2 n-1}(\widetilde{X}, \stackrel{\downarrow}{\widetilde{S}} ; \mathbb{Z}(1-n)) \rightarrow \operatorname{Div} \frac{0}{S}(\bar{X}, Y) \rightarrow 0
\end{aligned}
$$

where the middle vertical mapping is obtained as follows. By Lemma 2.4.2 we have

$$
H^{1}(\bar{X}-Z, Y ; \mathbb{Z}(1)) \cong H_{2 n-1}(\bar{X}-Y, Z ; \mathbb{Z}(1-n)) .
$$

Since $\widetilde{X}=\bar{X}-Y$ and $Z \hookrightarrow \widetilde{S}$ we have the following canonical map of mixed Hodge structures

$$
H_{2 n-1}(\bar{X}-Y, Z ; \mathbb{Z}(1-n)) \hookrightarrow H_{2 n-1}(\tilde{X}, \widetilde{S} ; \mathbb{Z}(1-n)) .
$$

The claimed map is obtained by composition of the duality isomorphism and the latter inclusion. Thus the diagram (21) commutes by the functoriality assertion in Lemma 2.4.2.

By diagram chase on (21) one can then see that the image of $D$ under the mapping

$$
e: L_{X} \rightarrow J^{1}(\bar{X}, Y)
$$

is the image of $[D]$ under the extension class map

$$
\mathbb{Z}[Z]^{0} \rightarrow J^{1}(\bar{X}, Y)
$$

determined by the top row of (21). Thus (20) commutes by Lemma 2.4.1 part $d$ ). The Theorem 2.4.5 is proved.

REMARK 2.4.6. - In order to show that (20) commutes, which is the key point in proving Theorem 2.4.5, one can instead choose $Z$ to be the union of all compact components of $\widetilde{S}$. By excision and duality we then have

$$
\begin{aligned}
H_{2 n-1}(X) \hookrightarrow H_{2 n-1}(X, S, \mathbb{Z}(1-n)) & \cong H_{2 n-1}(\widetilde{X}, \widetilde{S}, \mathbb{Z}(1-n)) \\
& \cong H_{2 n-1}(\widetilde{X}, Z, \mathbb{Z}(1-n)) \cong H^{1}(\bar{X}-Z, Y, \mathbb{Z}(1))
\end{aligned}
$$

and, comparing with the Mayer-Vietoris sequence (16) we have the following pull-back diagram (obtained as above from Lemma 2.4.2)

$$
\begin{aligned}
0 & \rightarrow H^{1}(\bar{X}, Y, \mathbb{Z}(1)) \rightarrow H^{1}(\bar{X}-Z, Y, \mathbb{Z}(1)) \rightarrow \operatorname{Div}_{Z}^{0}(\bar{X}, Y) \rightarrow 0 \\
& \| \\
0 & \rightarrow H^{1}(\bar{X}, Y, \mathbb{Z}(1)) \rightarrow H_{2 n-1}(X, \mathbb{Z}(1-n)) \rightarrow \operatorname{Div}_{S}^{0}{ }_{S}(\bar{X}, Y) \rightarrow 0
\end{aligned}
$$

in the category of mixed Hodge structures. Therefore, the claimed commutativity of (20), now follows directly from Lemma 2.4.1 part $e$ ), as $H^{1}(\bar{X}-Z, Y, \mathbb{Z}(1))$ is the Hodge realization of $\mathrm{Pic}^{+}(\bar{X}-Z, Y)$ and $\mathrm{Pic}^{-}(X)$ is a sub-1-motive. 


\section{5. Étale realization of $\mathrm{Pic}^{-}$}

Let $V$ be any $k$-scheme over a field $k$ (of characteristic zero). For any pair $(V, Z)$ where $Z$ is a closed subscheme of $V$ we denote by $i: Z \hookrightarrow V$ and $j: V-Z \hookrightarrow V$ the corresponding inclusions. We then have that $\mathbb{G}_{m, V} \rightarrow i_{*} \mathbb{G}_{m, Z}$ is an epimorphism of étale sheaves, and we let $\mathbb{G}_{m, V, Z)}$ denote its kernel. Associated to any such pair there is an exact sequence

$$
0 \rightarrow j_{!}\left(\mu_{m}\right) \rightarrow \mathbb{G}_{m,(V, Z)} \stackrel{m}{\longrightarrow} \mathbb{G}_{m,(V, Z)} \rightarrow 0
$$

induced by multiplication by $m$ on $\mathbb{G}_{m}$ 's and the snake lemma, where as usual $\mu_{m}$ denotes the étale sheaf of $m^{\text {th }}$ roots of unity $(c f .[\mathbf{3 3}],[\mathbf{5 2}])$. A "relative Hilbert's theorem 90 " is clearly available ( $c f$. [52, Section 1]).

Proposition 2.5.1. - There is an isomorphism

$$
H_{e ́ t}^{1}\left(V, \mathbb{G}_{m,(V, Z)}\right) \cong \operatorname{Pic}(V, Z)
$$

Proof. - From the Leray spectral sequence along $\varepsilon: V_{\text {ét }} \rightarrow V_{\text {Zar }}$ for the sheaf $\mathbb{G}_{m,(V, Z)}$ we get a functorial map $\operatorname{Pic}(V, Z) \rightarrow H_{\text {ét }}^{1}\left(V, \mathbb{G}_{m,(V, Z)}\right)$. We can then consider the long exact sequence (5) and compare with the corresponding sequence of étale cohomology groups. Since $H_{\text {ét }}^{i}\left(V, \mathbb{G}_{m, V}\right) \cong H^{i}\left(V, \mathcal{O}_{V}^{*}\right)$ and $H_{\text {ét }}^{i}\left(Z, \mathbb{G}_{m, Z}\right) \cong H^{i}\left(Z, \mathcal{O}_{Z}^{*}\right)$ for $i=0,1$ we then get the result.

Proposition 2.5.2. - We have the following "relative Kummer sequence"

$$
0 \rightarrow H^{0}\left(V, \mathbb{G}_{m,(V, Z)}\right) / m \stackrel{u}{\longrightarrow} H_{e ́ t}^{1}\left(V, j_{!}\left(\mu_{m}\right)\right) \stackrel{p}{\longrightarrow} \operatorname{Pic}(V, Z)_{m-\text { tors }} \rightarrow 0
$$

where:

- $H_{e ̂ t}^{1}\left(V, j_{!}\left(\mu_{m}\right)\right)$ can be interpreted as the group of isomorphism classes of triples $(\mathcal{L}, \varphi, \eta)$ given by a line bundle $\mathcal{L}$ on $V$, a trivialization $\varphi:\left.\mathcal{L}\right|_{Z} \cong \mathcal{O}_{Z}$ and an isomorphism $\eta: \mathcal{O}_{V} \cong \mathcal{L}^{\otimes m}$ which is compatible with $\varphi^{\otimes m}$, i.e., such that $\left.\eta\right|_{Z}=\varphi^{\otimes m}$

- $H^{0}\left(V, \mathbb{G}_{m,(V, Z)}\right)$ is the subgroup of those elements in $H^{0}\left(V, \mathcal{O}_{V}^{*}\right)$ yielding 1 in the group $H^{0}\left(Z, \mathcal{O}_{Z}^{*}\right)$;

- the map $u$ is defined by taking a unit $a \in H^{0}\left(V, \mathbb{G}_{m,(V, Z)}\right)$ to $\left(\mathcal{O}_{V}, 1, a^{-1}\right)$;

- the map $p$ takes a triple $(\mathcal{L}, \varphi, \eta)$ to the pair $(\mathcal{L}, \varphi)$ which is an $m$-torsion element of $\operatorname{Pic}(V, Z)$.

Proof. - The description above can be easily obtained by modifying the original argument for absolute Pic (cf. [33, III.4]).

We can regard $H_{\text {êt }}^{*}\left(V, j_{!}\left(\mu_{m}\right)\right)$ as "relative étale cohomology" groups of the pair $(V, Z)$ for which we adopt the notation $H_{\text {et }}^{*}\left(V, Z ; \mu_{m}\right)$.

Étale homology groups

$$
H_{*}^{\text {ét }}\left(V, \mu_{m}^{\otimes t}\right)
$$


are defined, for an $n$-dimensional $V$ and integer $t$, to be the cohomology groups of the following (dual) complex

$$
\mathrm{RHom}\left(\mathrm{R} \Gamma\left(V, \mu_{m}^{\otimes-t}\right), \mu_{m}^{\otimes(-n)}[-2 n]\right)
$$

in the (twisted) derived category of étale sheaves of $\mathbb{Z} / m$-modules; these homology groups, in general, are not the same as Borel-Moore étale homology groups defined by the dualizing complex. We then have the following result (cf. Lemma 2.4.2).

Lemma 2.5.3. - Let $V$ be an n-dimensional proper smooth variety over $k=\bar{k}$ of characteristic 0 . Let $A+B$ be a normal crossing divisor in $V$ such that $A \cap B=\varnothing$. Then there is a functorial duality isomorphism

$$
H_{e ́ t}^{r}\left(V-A, B ; \mu_{m}\right) \cong H_{2 n-r}^{e ́ t}\left(V-B, A ; \mu_{m}^{\otimes(1-n)}\right)
$$

which is compatible with Poincaré-Lefschetz duality.

Proof. - The same proof of Lemma 2.4.2 applies here to the étale sheaf $\mu_{m}$.

Suppose that $\bar{X}$ is a good n.c. compactification, with boundary $Y$, of a resolution $\tilde{X}$ of an equidimensional $n$-dimensional $k$-variety $X$, where $k=\bar{k}$, char. $k=0$, and let $S, \widetilde{S}, \bar{S}$ be as before. Let $(D, \mathcal{L}) \in \operatorname{Div}_{\bar{S} / S}(\bar{X}, Y) \times \operatorname{Pic}^{0}(\bar{X}, Y)$; by definition (see Chapter 1 for details)

$$
T_{\mathbb{Z} / m}\left(\operatorname{Pic}^{-}(X)\right)=\frac{\left\{(D, \mathcal{L}) \mid \eta_{D}: \mathcal{L}^{\otimes m} \cong \mathcal{O}_{\bar{X}}(-D)\right\}}{\left\{\left(m D, \mathcal{O}_{\bar{X}}(-D)\right)\right\}} .
$$

We have a canonical map

$$
\rho_{m}^{-}: T_{\mathbb{Z} / m}\left(\operatorname{Pic}^{-}(X)\right) \rightarrow H_{2 n-1}^{\text {ét }}\left(X, \mu_{m}^{\otimes(1-n)}\right)
$$

defined as follows. Let $D$ be a divisor in $\operatorname{Div} \frac{0}{S} / S(\bar{X}, Y)$ and let $Z$ be its support. If $(D, \mathcal{L})$ is a pair in $T_{\mathbb{Z} / m}\left(\mathrm{Pic}^{-}(X)\right)$ then $\left(\left.\mathcal{L}\right|_{\bar{X}-Z}, 1,\left.\eta_{D}\right|_{\bar{X}-Z}\right)$ belongs to $H_{\text {êt }}^{1}\left(\bar{X}-Z, Y ; \mu_{m}\right)$ by relative Kummer theory (i.e., the description in Proposition 2.5.2); furthermore, the image of the triple $\left(\left.\mathcal{L}\right|_{\bar{X}-Z}, 1,\left.\eta_{D}\right|_{\bar{X}-Z}\right)$ under the boundary map

$$
H_{\text {êt }}^{1}\left(\bar{X}-Z, Y, \mu_{m}\right) \rightarrow H_{\text {ét }, Z}^{2}\left(\bar{X}, Y, \mu_{m}\right)
$$

is the class of $D$, which vanishes, since by choice $D \in \operatorname{Div} \frac{0}{S} / S(\bar{X}, Y)$.

We have the following commutative diagram with exact rows

$$
\begin{aligned}
& 0 \rightarrow H_{\text {êt }}^{1}\left(\bar{X}, Y, \mu_{m}\right) \rightarrow H_{\text {êt }}^{1}\left(\bar{X}-Z, Y, \mu_{m}\right) \rightarrow H_{\text {êt }, Z}^{2}\left(\bar{X}, Y, \mu_{m}\right) \\
& 0 \rightarrow H_{2 n-1}^{\text {ét }}\left(\widetilde{X}, \mu_{m}^{\otimes 1-n}\right) \rightarrow H_{2 n-1}^{\text {ét }}\left(\widetilde{X}, \widetilde{S}, \mu_{m}^{\otimes 1-n}\right) \rightarrow H_{2 n-2}^{\text {ét }}\left(\widetilde{S}, \mu_{m}^{\otimes 1-n}\right) \\
& \downarrow \quad \downarrow \cong \downarrow \\
& 0 \rightarrow H_{2 n-1}^{\text {ét }}\left(X, \mu_{m}^{\otimes 1-n}\right) \rightarrow H_{2 n-1}^{\text {ét }}\left(X, S, \mu_{m}^{\otimes 1-n}\right) \rightarrow H_{2 n-2}^{\text {ét }}\left(S, \mu_{m}^{\otimes 1-n}\right)
\end{aligned}
$$

We then can define $\rho_{m}^{-}(D, \mathcal{L})$ to be the image of $\left(\left.\mathcal{L}\right|_{\bar{X}-Z}, 1,\left.\eta_{D}\right|_{\bar{X}-Z}\right)$ in

$$
H_{2 n-1}^{\text {ét }}\left(X, \mu_{m}^{\otimes(1-n)}\right) \text {. }
$$


We let

We can show the following.

$$
H_{2 n-1}^{\text {ét }}(X, \widehat{\mathbb{Z}}(1-n)) \stackrel{\text { def }}{=} \underset{m}{\lim _{m}} H_{2 n-1}^{\text {ét }}\left(X, \mu_{m}^{\otimes(1-n)}\right) .
$$

THEOREM 2.5.4. - Let $X$ be a variety of dimension $n$ over an algebraically closed field $k$ of characteristic zero. Then

$$
\widehat{T}\left(\operatorname{Pic}^{-}(X)\right) \cong H_{2 n-1}^{e ́ t}(X, \widehat{\mathbb{Z}}(1-n)) .
$$

Proof. - As in the proof of Theorem 2.4.5, we reduce immediately to the case when $X$ is equidimensional. Now we fix a choice of resolution $f: \widetilde{X} \rightarrow X$, good compactification $\bar{X}$, etc.

By definition, $\operatorname{Pic}^{-}(X)$ is given by the 1 -motive $\left[\operatorname{Div} \frac{0}{S} / S(\bar{X}, Y) \rightarrow \operatorname{Pic}^{0}(\bar{X}, Y)\right]$. We have the following commutative diagram

$$
\begin{aligned}
& 0 \rightarrow \widehat{T}\left(\operatorname{Pic}^{0}(\bar{X}, Y)\right) \rightarrow \widehat{T}\left(\operatorname{Pic}^{-}(X)\right) \quad \rightarrow \widehat{T}\left(\operatorname{Div} \frac{0}{S} / S(\bar{X}, Y)[1]\right) \rightarrow 0 \\
& \bar{\rho}_{\text {ét }} \downarrow \quad \downarrow \rho_{\text {ét }} \quad \downarrow \rho_{\text {ét }}^{0} \\
& 0 \rightarrow H_{\text {ét }}^{1}(\bar{X}, Y ; \widehat{\mathbb{Z}}(1)) \rightarrow H_{2 n-1}^{\text {ét }}(X, \widehat{\mathbb{Z}}(1-n)) \rightarrow \quad \widehat{\mathbb{Z}}^{\oplus r} \quad \rightarrow 0
\end{aligned}
$$

where the bottom row is given by the Mayer-Vietoris sequence for étale homology and the duality Lemma 2.5.3 ( $r$ is a certain non-negative integer), and the top exact sequence is given by (3) in Chapter 1 . We get the mapping $\rho_{\text {ét }}$ above by taking limit of $\rho_{m}^{-}$, and $\bar{\rho}_{\text {ét }}$ is the induced map.

Note that $\bar{\rho}_{\text {ét }}$ may be viewed as the analog of $\rho_{\text {ét }}$ for the variety $\widetilde{X}$. It is also easy to see from the definitions that $\widehat{T}\left(\operatorname{Div} \frac{0}{S} / S(\bar{X}, Y)[1]\right) \cong \widehat{\mathbb{Z}}^{\oplus r}$ as well, such that $\rho_{\text {ét }}^{0}$ is an isomorphism. Granting this, we are left to show our claim holds true for smooth schemes, i.e., that $\bar{\rho}_{\text {ét }}$ is an isomorphism. The latter follows from the fact that the relative Néron-Severi group of $(\bar{X}, Y)$ is finitely generated, whence $\widehat{T}\left(\operatorname{Pic}^{0}(\bar{X}, Y)\right)=\lim _{m} \operatorname{Pic}(\bar{X}, Y)_{m-\text { tors }}$, and, by Proposition 2.5.1 and the Kummer sequence in Proposition 2.5.2, we have $\operatorname{Pic}(\bar{X}, Y)_{m-\text { tors }}=H_{\text {ét }}^{1}\left(\bar{X}, Y ; \mu_{m}\right)$, since $H^{0}\left(X, \mathbb{G}_{m,(\bar{X}, Y)}\right)$ is divisible.

REMARK 2.5.5. - Theorem 2.5.4 can also be used to show that $\mathrm{Pic}^{-}$is independent of the choices of resolutions and compactifications. In fact, after Proposition 1.3.1, the induced isomorphism on étale realizations lifts to 1-motives. But, as remarked before, we consider this proof to be "not in the spirit of the theory of 1-motives".

\subsection{De Rham realization of $\mathrm{Pic}^{-}$}

Let $k$ be a field of characteristic 0 . Let $\bar{X}$ be a smooth $k$-variety, with smooth compactification $\bar{X}$ and normal crossing boundary $Y=\bar{X}-X$. Let $\pi: \widetilde{Y} \rightarrow Y$ be the normalization, and $i: Y \hookrightarrow \bar{X}$ the inclusion. Note that $\tilde{Y}$ is a smooth proper $k$-variety as well. 
If $(\mathcal{L}, \nabla)$ is a line bundle on $\bar{X}$ equipped with an integrable ( $k$-linear) connection, then restriction to $Y$ yields a connection on $\left.\mathcal{L}\right|_{Y}$ with values in $\pi_{*} \Omega_{\widetilde{Y}}^{1}$,

$$
\widetilde{\nabla}: i^{*} \mathcal{L} \rightarrow i^{*} \mathcal{L} \otimes \mathcal{O}_{Y} \pi_{*} \Omega_{\widetilde{Y}}^{1}
$$

defined as the composition of the restricted connection $i^{*} \mathcal{L} \rightarrow i^{*} \mathcal{L} \otimes_{\mathcal{O}_{Y}} \Omega_{Y}^{1}$ with the natural $\mathcal{O}_{Y}$-linear map $i^{*} \mathcal{L} \otimes \Omega_{Y}^{1} \rightarrow i^{*} \mathcal{L} \otimes \pi_{*} \Omega_{\widetilde{Y}}^{1}$. There is also a connection $\widetilde{d}: \mathcal{O}_{Y} \rightarrow \pi_{*} \Omega_{\widetilde{Y}}^{1}$, similarly defined using the exterior derivative map $d: \mathcal{O}_{Y} \rightarrow \Omega_{Y}^{1}$; this is just $\widetilde{\nabla}$ in the case when $\mathcal{L}=\mathcal{O}_{\bar{X}}$ and $\nabla=d$.

We will denote by $\operatorname{Pic}^{\natural}(\bar{X}, Y)$ the group of isomorphism classes of triples $(\mathcal{L}, \nabla, \varphi)$, where $(\mathcal{L}, \nabla)$ is a line bundle on $\bar{X}$ with an integrable connection and

$$
\varphi:\left(i^{*} \mathcal{L}, \widetilde{\nabla}\right) \cong\left(\mathcal{O}_{Y}, \widetilde{d}\right)
$$

is a trivialization on $Y$ as connections with values in $\pi_{*} \Omega_{\widetilde{Y}}^{1}$; equivalently, we have a trivialization of $i^{*} \mathcal{L}$ such that the induced trivialization of $\pi^{*} i^{*} \mathcal{L}$ is given by a flat section, for the induced connection on $\pi^{*} i^{*} \mathcal{L}$ (in the standard sense) obtained from $\nabla$.

We can consider the relative $\downarrow$-Picard functor on the category of schemes over $k$, which we denote by $\operatorname{Pic}_{(\bar{X}, Y) / k}^{\natural}$, and is defined to be the $f p q c$-sheaf associated to the functor

$$
T \longmapsto \operatorname{Pic}^{\natural}\left(\bar{X} \times_{k} T, Y \times_{k} T\right) .
$$

We clearly have the following commutative square

$$
\begin{gathered}
\operatorname{Pic}^{\natural}(\bar{X}, Y) \rightarrow \operatorname{Pic}^{\natural}(\bar{X}) \\
\downarrow \\
\operatorname{Pic}(\bar{X}, Y) \rightarrow \operatorname{Pic}(\bar{X})
\end{gathered}
$$

which is functorial as well.

Let $i: Y \hookrightarrow \bar{X}$ be the inclusion of the normal crossing boundary, and let $\pi: \tilde{Y} \rightarrow Y$ be the normalization. We have an induced relative dlog map given by the following diagram

$$
\begin{aligned}
& 0 \rightarrow \Omega \frac{1}{X}(\log Y)(-Y) \rightarrow \quad \Omega \frac{1}{X} \quad \rightarrow i_{*} \pi_{*} \Omega_{\widetilde{Y}}^{1} \rightarrow 0 \\
& \uparrow \operatorname{dlog} \quad \uparrow \operatorname{dlog} \uparrow \operatorname{dlog} \\
& 0 \rightarrow \mathcal{O}_{(\bar{X}, Y)}^{*} \quad \rightarrow \quad \mathcal{O}_{\bar{X}}^{*} \quad \rightarrow \quad i_{*} \mathcal{O}_{Y}^{*} \rightarrow 0
\end{aligned}
$$

We now have the following.

Proposition 2.6.1. - Let $(\bar{X}, Y)$ be any pair as above over $k=\bar{k}$, and let $Y_{i}(i=$ $1,2, \ldots)$ denote the (smooth) irreducible components of the normal crossing boundary divisor $Y$.

a) There is a functorial isomorphism

$$
\operatorname{Pic}^{\natural}(\bar{X}, Y) \cong \mathbb{H}^{1}\left(\bar{X}, \mathcal{O}_{(\bar{X}, Y)}^{*} \stackrel{\operatorname{dlog}}{\longrightarrow} \Omega \frac{1}{X}(\log Y)(-Y)\right) .
$$


b) There is an extension

$$
1 \rightarrow \frac{H^{0}\left(Y, \mathcal{O}_{Y}^{*}\right)}{H^{0}\left(\bar{X}, \mathcal{O}_{\bar{X}}^{*}\right)} \rightarrow \operatorname{Pic}^{\natural}(\bar{X}, Y)^{0} \rightarrow \operatorname{ker}^{0}\left(\operatorname{Pic}^{\natural}(\bar{X})^{0} \rightarrow \oplus \operatorname{Pic}^{\natural}\left(Y_{i}\right)^{0}\right) \rightarrow 0
$$

where $\left(\mathrm{Pic}^{\natural}\right)^{0}$ denotes the pull-back of $\mathrm{Pic}^{0}$ in $\mathrm{Pic}^{\natural}$.

c) The universal $\mathbb{G}_{a}$-extension of the semi-abelian variety $\operatorname{Pic}^{0}(\bar{X}, Y)$ is given by the $k$-group scheme $\left(\operatorname{Pic}_{(\bar{X}, Y) / k}^{\natural}\right)^{0}$, i.e., in the notation of Section 1.4, we have an isomorphism

$$
\left(\operatorname{Pic}^{0}(\bar{X}, Y)\right)^{\natural} \cong\left(\operatorname{Pic}_{(\bar{X}, Y) / k}^{\natural}\right)^{0} .
$$

d) We have an isomorphism

$$
\text { Lie } \operatorname{Pic}^{\natural}(\bar{X}, Y)^{0} \cong \mathbb{H}^{1}\left(\bar{X}, \mathcal{O}_{\bar{X}}(-Y) \rightarrow \Omega \frac{1}{X}(\log Y)(-Y)\right) .
$$

Proof. - In order to show part a) we consider the canonical mapping which associates to any line bundle $\mathcal{L}$ with an integrable connection $\nabla$, trivialized along $Y$ (in the appropriate sense), the cohomology class of a Čech cocycle given by the transition functions defining $\mathcal{L}$ and the induced forms. Since the following sequence (defined by the obvious maps)

$0 \rightarrow H^{0}\left(\bar{X}, \Omega \frac{1}{X}(\log Y)(-Y)\right) \rightarrow \operatorname{Pic}^{\natural}(\bar{X}, Y) \rightarrow \operatorname{Pic}(\bar{X}, Y) \rightarrow H^{1}\left(\bar{X}, \Omega \frac{1}{X}(\log Y)(-Y)\right)$ is exact, we get the claimed isomorphism: note that

$$
H^{0}\left(\bar{X}, \Omega_{\bar{X}}^{1}(\log Y)(-Y)\right) \subset H^{0}\left(\bar{X}, \Omega \frac{1}{X}\right)
$$

consists of closed 1-forms, since char. $k=0$.

The exact sequence in $b$ ) is obtained by the exact sequence of complexes given by the columns in (23): in fact, the following equation holds

$$
\operatorname{ker}^{0}\left(\operatorname{Pic}^{\natural}(\bar{X})^{0} \rightarrow \mathbb{H}^{1}\left(Y, \mathcal{O}_{Y}^{*} \rightarrow \pi_{*} \Omega_{\widetilde{Y}}^{1}\right)\right)=\operatorname{ker}^{0}\left(\operatorname{Pic}^{\natural}(\bar{X})^{0} \rightarrow \oplus \operatorname{Pic}^{\natural}\left(Y_{i}\right)^{0}\right)
$$

by the Proposition 2.1.2.

From the above discussion we get the following diagram with short exact rows and columns

$$
\begin{aligned}
& 0
\end{aligned}
$$

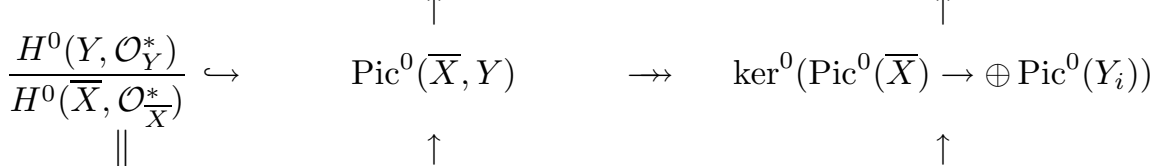

$$
\begin{aligned}
& \frac{H^{0}\left(Y, \mathcal{O}_{Y}^{*}\right)}{H^{0}\left(\bar{X}, \mathcal{O}_{\frac{\pi}{X}}^{*}\right)} \hookrightarrow \quad \operatorname{Pic}^{\natural}(\bar{X}, Y)^{0} \quad \rightarrow \quad \operatorname{ker}^{0}\left(\operatorname{Pic}^{\natural}(\bar{X})^{0} \rightarrow \oplus \operatorname{Pic}^{\natural}\left(Y_{i}\right)^{0}\right) \\
& H^{0}\left(\bar{X}, \Omega_{\bar{X}}(\log Y)(-Y)\right)=\operatorname{ker}\left(H^{0}\left(\bar{X}, \Omega_{\bar{X}}\right) \underset{\uparrow}{\stackrel{\uparrow}{\rightarrow}} \oplus_{i} H^{0}\left(Y_{i}, \Omega_{Y_{i}}^{1}\right)\right)
\end{aligned}
$$


Therefore we see that $\operatorname{Pic}^{\natural}(\bar{X}, Y)^{0}$ is the group of $k$-points of the pull-back of the group scheme

$$
\operatorname{ker}^{0}\left(\left(\operatorname{Pic}_{\bar{X} / k}^{\natural}\right)^{0} \rightarrow \oplus\left(\operatorname{Pic}_{Y_{i} / k}^{\natural}\right)^{0}\right) .
$$

The latter is the universal extension of the abelian variety

$$
\operatorname{ker}^{0}\left(\operatorname{Pic}^{0}(\bar{X}) \rightarrow \oplus \operatorname{Pic}^{0}\left(Y_{i}\right)\right),
$$

therefore $\operatorname{Pic}^{\natural}(\bar{X}, Y)^{0}$ is the universal extension of the semi-abelian variety $\operatorname{Pic}^{0}(\bar{X}, Y)$, and $c$ ) is proved.

The part $d$ ) is standard (e.g., can be obtained in a manner similar to the corresponding result for the usual Picard functors, by computing $k[\varepsilon]$-points as in [32]).

Let $X$ be equidimensional over $k=\bar{k}$, and fix a resolution $f: \widetilde{X} \rightarrow X$ and good normal crossing compactification $\bar{X}$ with boundary $Y$, as usual. Let $Z$ denote the union of all compact components in $\widetilde{S}$. By our choice of resolution and compactification, $Z$ has normal crossings, and $Z \cap Y=\varnothing$. Denote by $Z_{j}(j=1,2, \ldots)$ its smooth irreducible components. Recall (Definition 2.2.1) that $Z$ yields a 1-motive defined as follows

$$
\operatorname{Pic}^{+}(\bar{X}-Z, Y) \stackrel{\text { def }}{=}\left[\operatorname{Div}_{Z}^{0}(\bar{X}, Y) \stackrel{u_{Z}}{\longrightarrow} \operatorname{Pic}^{0}(\bar{X}, Y)\right] .
$$

By definition, $\operatorname{Pic}^{-}(X)$ is a sub-1-motive of $\operatorname{Pic}^{+}(\bar{X}-Z, Y)$.

Correspondingly, we define the group $\operatorname{Pic}^{\natural-\log }(\bar{X}-Z, Y)$ as the group of isomorphism classes of triples $\left(\mathcal{L}, \nabla^{\log }, \varphi\right)$ where $\mathcal{L}$ is a line bundle on $\bar{X}, \nabla^{\log }$ is an integrable connection on $\mathcal{L}$ with $\log$ poles along $Z$, i.e.,

$$
\nabla^{\log }: \mathcal{L} \rightarrow \mathcal{L} \otimes \Omega \frac{1}{X}(\log Z)
$$

and $\varphi:\left(i^{*} \mathcal{L}, \widetilde{\nabla}\right) \cong\left(\mathcal{O}_{Y}, \widetilde{d}\right)$ is a trivialization (note that we are assuming $Z \cap Y=\varnothing$ ).

We also have the following commutative square

$$
\begin{aligned}
\operatorname{Pic}^{\natural}(\bar{X}, Y) & \rightarrow \operatorname{Pic}^{\natural-\log }(\bar{X}-Z, Y) \\
\downarrow & \downarrow \\
\operatorname{Pic}(\bar{X}, Y) & =\operatorname{Pic}(\bar{X}, Y)
\end{aligned}
$$

which is functorial. Denote $\operatorname{Pic}^{\natural-\log }(\bar{X}-Z, Y)^{0}$ the pull-back of $\operatorname{Pic}^{0}(\bar{X}, Y) \subseteq$ $\operatorname{Pic}(\bar{X}, Y)$ along the right hand vertical map in the above diagram.

Our goal here is to construct the universal $\mathbb{G}_{a}$-extension $\operatorname{Pic}^{+}(\bar{X}-Z, Y)^{\natural}$ of the 1-motive $\operatorname{Pic}^{+}(\bar{X}-Z, Y)$. In order to do this, consider the following lifting

$$
u_{Z}^{\natural}: \operatorname{Div}_{Z}^{0}(\bar{X}, Y) \rightarrow \operatorname{Pic}^{\natural-\log }(\bar{X}-Z, Y)^{0}
$$

of $u_{Z}: \operatorname{Div}_{Z}^{0}(\bar{X}, Y) \rightarrow \operatorname{Pic}^{0}(\bar{X}, Y)$. The lifting $u_{Z}^{\natural}$ is obtained from the fact that given a divisor $D \in \operatorname{Div}_{Z}(\bar{X}, Y)$, the line bundle $\mathcal{O}_{\bar{X}}(D)$ comes equipped also with a canonical connection with $\log$ poles along $\operatorname{supp}(D) \subset Z$. The connection is characterized by the property that the tautological meromorphic section, with divisor $D$, is flat (i.e., if $s$ is this section, $\nabla(s)=0$ defines a connection on the open complement of 
$\operatorname{supp}(D)$, which one verifies, by local calculation, has a unique meromorphic extension with $\log$ poles along $\operatorname{supp}(D)$ ). We then have the following result.

Lemma 2.6.2. - Let $\bar{X}, Y$ and $Z$ be as above.

a) There is an isomorphism

$$
\operatorname{Pic}^{\natural-\log }(\bar{X}-Z, Y) \cong \mathbb{H}^{1}\left(\bar{X}, \mathcal{O}_{(\bar{X}, Y)}^{*} \stackrel{\operatorname{dlog}}{\longrightarrow} \Omega \frac{1}{X}(\log (Y+Z))(-Y)\right) .
$$

b) The group $\operatorname{Pic}^{\natural-\log }(\bar{X}-Z, Y)^{0}$ is an extension of the following vector group

$$
\operatorname{Div}_{Z}^{0}(\bar{X}, Y)^{\natural}=\operatorname{ker}\left(\oplus_{j} H^{0}\left(Z_{j}, \mathcal{O}_{Z_{j}}\right) \rightarrow H^{1}\left(\bar{X}, \Omega \frac{1}{X}(\log (Y))(-Y)\right)\right)
$$

by the group $\operatorname{Pic}^{\natural}(\bar{X}, Y)^{0}$.

c) We then have that

$$
\operatorname{Pic}^{+}(\bar{X}-Z, Y)^{\natural} \cong\left[\operatorname{Div}_{Z}^{0}(\bar{X}, Y) \stackrel{u_{Z}^{\natural}}{\longrightarrow} \operatorname{Pic}^{\natural-\log }(\bar{X}-Z, Y)^{0}\right] .
$$

d) We have an isomorphism

$$
\operatorname{LiePic}{ }^{\natural-\log }(\bar{X}-Z, Y)^{0} \cong \mathbb{H}^{1}\left(\bar{X}, \mathcal{O}_{\bar{X}}(-Y) \rightarrow \Omega \frac{1}{X}(\log (Y+Z))(-Y)\right) .
$$

Proof. - The proofs of parts $a$ ) and b) are very similar to those in Proposition 2.6.1. In fact we have relative residue sequences given by the first row of the following commutative diagram $(c f .[\mathbf{2 0}, 2.3])$

$$
\begin{aligned}
& 0 \quad 0 \\
& \downarrow \quad \downarrow \\
& 0 \rightarrow \Omega \frac{1}{X}(\log Y)(-Y) \rightarrow \Omega \frac{1}{X}(\log (Y+Z))(-Y) \rightarrow \oplus_{j} \mathcal{O}_{Z_{j}}\left(-Z_{j} \cap Y\right) \rightarrow 0 \\
& 0 \rightarrow \quad \quad \Omega \frac{1}{X} \quad \rightarrow \quad \Omega \frac{1}{X}(\log Z) \quad \rightarrow \quad \oplus_{j} \mathcal{O}_{Z_{j}} \quad \rightarrow 0 \\
& \begin{array}{ccc}
\oplus_{i} \Omega_{Y_{i}}^{1} & \cong & \oplus_{i} \Omega_{Y_{i}}^{1}\left(\log Z \cap Y_{i}\right) \\
\downarrow & & \downarrow \\
0 & & 0
\end{array}
\end{aligned}
$$

where the isomorphisms are because $Y \cap Z=\varnothing$. Here recall that $Y_{i}$ are the irreducible components of $Y$. The following diagram (similar to (23))

$$
\begin{aligned}
& 0 \rightarrow \Omega \frac{1}{X}(\log Y)(-Y) \rightarrow \Omega \frac{1}{X}\left(\log (Y+Z)(-Y) \rightarrow \oplus_{j} \mathcal{O}_{Z_{j}} \rightarrow 0\right. \\
& \uparrow \operatorname{dlog} \uparrow \operatorname{dlog} \uparrow \\
& 0 \rightarrow \mathcal{O}_{(\bar{X}, Y)}^{*} \quad=\quad \mathcal{O}_{(\bar{X}, Y)}^{*} \quad \rightarrow \quad 0 \quad \rightarrow 0,
\end{aligned}
$$

together with (24), yield $a$ ) and the extension of

$$
\operatorname{Div}_{Z}^{0}(\bar{X}, Y)^{\natural}=\operatorname{ker}\left(\oplus_{j} H^{0}\left(Z_{j}, \mathcal{O}_{Z_{j}}\right) \rightarrow H^{1}\left(\bar{X}, \Omega \frac{1}{X}(\log Y)(-Y)\right)\right)
$$


claimed in $b$ ). In fact, from the above, we get the following push-out diagram

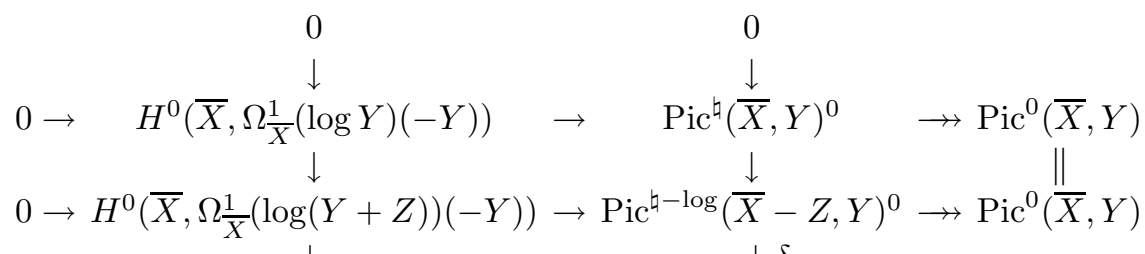

$$
\begin{aligned}
& \begin{array}{cc}
\operatorname{Div}_{Z}^{0}(\bar{X}, Y)^{\natural} & = \\
\downarrow & \operatorname{Div}_{Z}^{0}(\bar{X}, Y)^{\natural} \\
\downarrow & \downarrow \\
0 & 0
\end{array}
\end{aligned}
$$

where "res" is the ordinary residue of forms and $\delta$ is the residue of connections. Note that here $\operatorname{Pic}^{\natural-\log }(\bar{X}-Z, Y)^{0}$ surjects onto $\operatorname{Pic}^{0}(\bar{X}, Y)$ as the canonical map

$$
\operatorname{Pic}(\bar{X}, Y) \rightarrow H^{1}\left(\bar{X}, \Omega \frac{1}{X}(\log (Y+Z))(-Y)\right)
$$

factors through $H^{1}\left(\bar{X}, \Omega \frac{1}{X}(\log (Y))(-Y)\right)$.

For the latter claims $c$ ) and $d$ ) we proceed as follows. Considering the above extensions, we are left to show that the canonical induced map

$$
\operatorname{Ext}\left(\operatorname{Pic}^{+}(\bar{X}-Z, Y), \mathbb{G}_{a}\right)^{\vee} \stackrel{\cong}{\longrightarrow} H^{0}\left(\bar{X}, \Omega \frac{1}{X}(\log (Y+Z))(-Y)\right)
$$

is an isomorphism: in fact, granting $(25), c$ ) follows from the above push-out diagram, Proposition 2.6.1 and the construction of the universal extension as being given in Chapter 1.

In order to show that the map in (25) is an isomorphism, we consider the following commutative diagram

$$
\begin{array}{ccc}
0 & 0 \\
\downarrow & \downarrow \\
\operatorname{Ext}\left(\operatorname{Pic}^{0}(\bar{X}, Y), \mathbb{G}_{a}\right)^{\vee} & \stackrel{\cong}{\downarrow} & H^{0}\left(\bar{X}, \Omega_{\frac{1}{X}}(\log (Y))(-Y)\right) \\
\left.\operatorname{Ext}^{\downarrow} \operatorname{Pic}^{+}(\bar{X}-Z, Y), \mathbb{G}_{a}\right)^{\vee} & \rightarrow & H^{0}\left(\bar{X}, \Omega_{\frac{1}{X}}(\log (Y+Z))(-Y)\right) \\
\downarrow & & \downarrow \text { res } \\
\operatorname{Hom}\left(\operatorname{Div}_{Z}^{0}(\bar{X}, Y), \mathbb{G}_{a}\right)^{\vee} & \stackrel{\cong}{\longrightarrow} & \operatorname{Div}_{Z}^{0}(\bar{X}, Y)^{\natural} \\
\downarrow & & \downarrow \\
0 & & 0
\end{array}
$$

where: by Proposition 2.6.1 we know that

$$
\operatorname{Ext}\left(\operatorname{Pic}^{0}(\bar{X}, Y), \mathbb{G}_{a}\right)^{\vee} \cong H^{0}\left(\bar{X}, \Omega \frac{1}{X}(\log Y)(-Y)\right)
$$

and, since $\operatorname{Div}_{Z}(\bar{X}, Y)=\mathbb{Z}[Z]$, we have that

$$
\operatorname{Hom}\left(\mathbb{Z}[Z]^{0}, \mathbb{G}_{a}\right)^{\vee} \cong \operatorname{ker}\left(\oplus_{j} H^{0}\left(Z_{j}, \mathcal{O}_{Z_{j}}\right) \rightarrow H^{1}\left(\bar{X}, \Omega \frac{1}{X}(\log Y)(-Y)\right)\right)
$$


is the restriction of the canonical isomorphism $\operatorname{Hom}\left(\mathbb{Z}[Z], \mathbb{G}_{a}\right)^{\vee} \cong \oplus_{j} H^{0}\left(Z_{j}, \mathcal{O}_{Z_{j}}\right)$ to those divisors which are algebraically equivalent to zero relatively to $Y$.

Thus the relative residue sequence (24) yields that the map in (25) is an isomorphism as well. The Lie algebra computation yielding $d$ ) is then straightforward.

REMARK 2.6.3 (De Rham cohomology and homology). - For a variety $X$ over a field $k$ of characteristic zero (not necessarily algebraically closed), let $X . \rightarrow X$ be a smooth proper hypercovering, and let $X . \hookrightarrow \bar{X}$. be a smooth compactification with normal crossing boundary $Y_{\text {. }}$, as in [15]. We define the De Rham cohomology of $X$ as follows:

$$
H_{\mathrm{DR}}^{*}(X) \stackrel{\text { def }}{=} \mathbb{H}^{*}\left(\bar{X} \cdot, \Omega_{\bar{X}}^{\cdot}(\log Y \cdot)\right) .
$$

The Hodge-De Rham filtration is that induced by truncations, as usual. This definition is in accordance with Deligne's definition in $[\mathbf{1 5}, 10.3 .15]$, determining De Rham cohomology as a filtered vector space. Similarly, we define the Tate twist $H_{\mathrm{DR}}^{*}(X)(m)$ to be the underlying vector space of $H_{\mathrm{DR}}^{*}(X)$, with the obvious shift in indexing of its Hodge-De Rham filtration. Relative cohomology may also be defined in a similar way, as in [15] (see also [51]).

De Rham homology, denoted by $H_{*}^{D R}(X) \stackrel{\text { def }}{=} H_{\mathrm{DR}}^{*}(X)^{\vee}$, is defined to be the dual (filtered) vector space: it differs, in general, from Hartshorne (Borel-Moore) De Rham homology $[\mathbf{2 9}]$.

It can be shown, by comparison with the case $k=\mathbb{C}$ and cohomological descent, that

(i) the underlying $k$-vector space of $H_{\mathrm{DR}}^{*}(X)$ is naturally identified with Hartshorne's algebraic De Rham cohomology [29]

(ii) $\left(H_{\mathrm{DR}}^{*}(X), F^{*}\right)$ is independent of the choice of the hypercovering and compactification $X . \hookrightarrow \bar{X}$.

(iii) if $f: X \rightarrow Y$ is a morphism of $k$-varieties, the induced map

$$
f^{*}: H_{\mathrm{DR}}^{*}(Y) \rightarrow H_{\mathrm{DR}}^{*}(X)
$$

is strictly compatible with the respective Hodge-De Rham filtrations; in particular, if the underlying linear transformation is an isomorphism of vector spaces, then it is an isomorphism of filtered vector spaces

(iv) if $\operatorname{dim} X=n$, then $H_{\mathrm{DR}}^{i}(X)=0$ for $i>2 n$, and for irreducible $X$ over $k=\bar{k}$, $H_{\mathrm{DR}}^{2 n}(X)$ is either 0 (if $X$ is not proper over $k$ ) or 1-dimensional; if $X^{(n)}$ is the union of the $n$-dimensional irreducible components of $X$, then

$$
H_{\mathrm{DR}}^{i}(X) \rightarrow H_{\mathrm{DR}}^{i}\left(X^{(n)}\right)
$$

is an isomorphism for $i \geqslant 2 n-1$

(v) $H_{\mathrm{DR}}^{*}$ has other standard properties, like the excision isomorphism, and the Mayer-Vietoris exact sequence; these are valid in the category of filtered vector spaces and strictly compatible linear maps. 
If $X$ is smooth over $k$, and $\bar{X}$ is a smooth compactification with normal crossing boundary $Y$, we may regard $\bar{X}$ and $Y$ as "constant" simplicial schemes, so that we obtain

$$
H_{\mathrm{DR}}^{*}(X)=\mathbb{H}^{*}\left(\bar{X}, \Omega_{\bar{X}}^{\cdot}(\log Y)\right) .
$$

More generally, if $\bar{X}$ is a proper smooth $k$-variety and $Y, Z$ are disjoint normal crossing divisors, we get

$$
H_{\mathrm{DR}}^{*}(\bar{X}-Z, Y) \stackrel{\text { def }}{=} \mathbb{H}^{*}\left(\bar{X}, \Omega_{\bar{X}}(\log (Y+Z))(-Y)\right)
$$

with Hodge-De Rham filtration defined by truncation of the (twisted) log De Rham complex.

Now Lemma 2.6.2 implies the following.

Corollary 2.6.4. - Let $\bar{X}$ be a non-singular proper $k$-variety, $Z$ and $Y$ disjoint normal crossing divisors in $\bar{X}$. Then there is a natural isomorphism of filtered $k$-vector spaces

$$
T_{\mathrm{DR}}\left(\mathrm{Pic}^{+}(\bar{X}-Z, Y)\right) \cong H_{\mathrm{DR}}^{1}(\bar{X}-Z, Y)(1) .
$$

We now have the following duality result.

LEMMA 2.6.5. - Let $V$ be an n-dimensional proper smooth algebraic variety over a field of characteristic zero. Let $A+B$ be a normal crossing divisor in $V$ such that $A \cap B=\varnothing$. Then there is a functorial duality isomorphism

$$
H_{\mathrm{DR}}^{r}(V-A, B)(-n) \cong H_{2 n-r}^{D R}(V-B, A)
$$

which is compatible with the Hodge-De Rham filtration and Poincaré-Lefschetz duality.

Proof. — We can consider the following pairing

$$
\begin{gathered}
\Omega_{V}^{\cdot}(\log (A+B))(-A) \otimes \Omega_{V}^{\cdot}(\log (A+B))(-B) \\
\downarrow \\
\Omega_{V}^{\cdot}(\log (A+B))(-A-B) \\
\downarrow \\
\Omega_{V}^{\cdot}
\end{gathered}
$$

It will suffices to show that such a pairing yields non-degenerate pairings on hypercohomology

$$
\mathbb{H}^{r}\left(V, \Omega_{V}^{\cdot}(\log (A+B))(-A)\right) \otimes_{k} \mathbb{H}^{2 n-r}\left(V, \Omega_{V}(\log (A+B))(-B)\right) \rightarrow \mathbb{H}^{2 n}\left(V, \Omega_{V}\right)
$$

where $\mathbb{H}^{2 n}\left(V, \Omega_{V}^{\cdot}\right)=H^{n}\left(V, \Omega_{V}^{n}\right)=k$.

Since we are in characteristic zero we are left to show it for $k=\mathbb{C}$ for which it is clear from the proof of Lemma 2.4.2 and Remark 2.4.4. Alternately, one can deduce the duality isomorphism, as in the proof of Poincaré duality for algebraic De Rham cohomology, by reducing to Serre duality (cf. [28, III.8]). 
THEOREM 2.6.6. - Let $X$ be any $n$-dimensional $k$-variety, where $k$ is algebraically closed of characteristic 0 . Then

$$
T_{\mathrm{DR}}\left(\mathrm{Pic}^{-}(X)\right) \cong H_{2 n-1}^{D R}(X)(1-n) .
$$

Proof. - As usual, we can reduce to the case when $X$ is equidimensional. Fix a resolution $f: \widetilde{X} \rightarrow X$ with good normal crossing compactification $\bar{X}$ and boundary $Y$.

As above, let $Z$ be the union of all compact components of $\widetilde{S}$. We clearly have the following (see (24)) relative residue sequence

$$
0 \rightarrow \Omega_{\frac{1}{X}}(\log Y)(-Y) \rightarrow \Omega \frac{1}{X}(\log (Y+Z))(-Y) \rightarrow \oplus_{j} \mathcal{O}_{Z_{j}} \rightarrow 0
$$

where $Z_{j}$ are the smooth irreducible components of $Z$. Moreover

$$
\begin{aligned}
H_{2 n-1}^{D R}(X)(1-n) \hookrightarrow H_{2 n-1}^{D R}(X, S)(1-n) & \cong H_{2 n-1}^{D R}(\widetilde{X}, \widetilde{S})(1-n) \\
& \cong H_{2 n-1}^{D R}(\widetilde{X}, Z)(1-n) \cong H_{\mathrm{DR}}^{1}(\bar{X}-Z, Y)(1)
\end{aligned}
$$

by excision and duality, i.e., Lemma 2.6.5, and we have the following pull-back diagram (compare with (22))

$$
\begin{aligned}
& 0 \rightarrow H_{\mathrm{DR}}^{1}(\bar{X}, Y)(1) \rightarrow H_{\mathrm{DR}}^{1}(\bar{X}-Z, Y)(1) \stackrel{\text { res }}{\longrightarrow} \operatorname{Div}_{Z}^{0}(\bar{X}, Y) \otimes k \rightarrow 0 \\
& 0 \rightarrow H_{\mathrm{DR}}^{1}(\bar{X}, Y)(1) \rightarrow H_{2 n-1}^{D R}(X)(1-n) \rightarrow \operatorname{Div} \frac{0}{S / S}(\bar{X}, Y) \otimes k \rightarrow 0
\end{aligned}
$$

by duality and the Mayer-Vietoris sequence for De Rham homology.

Consider the following pull-back diagram of 1-motives

$$
\begin{aligned}
0 & \rightarrow \operatorname{Pic}^{0}(\bar{X}, Y) \rightarrow \operatorname{Pic}^{+}(\bar{X}-Z, Y) \rightarrow \operatorname{Div}_{Z}^{0}(\bar{X}, Y)[1] \rightarrow 0 \\
0 & \rightarrow \operatorname{Pic}^{0}(\bar{X}, Y) \rightarrow \operatorname{Pic}^{-}(X) \rightarrow \operatorname{Div} \frac{0}{S} / S(\bar{X}, Y)[1] \rightarrow 0 .
\end{aligned}
$$

We then get the following commutative diagram with short exact rows (and whose middle column implies the theorem)

$$
\begin{array}{ccccc}
T_{\mathrm{DR}}\left(\operatorname{Pic}^{0}(\bar{X}, Y)\right) & \hookrightarrow & T_{\mathrm{DR}}\left(\operatorname{Pic}^{+}(\bar{X}-Z, Y)\right) & \rightarrow & T_{\mathrm{DR}}\left(\operatorname{Div}_{Z}^{0}(\bar{X}, Y)[1]\right) \\
\cong \uparrow & & \uparrow & & \\
H_{\mathrm{DR}}^{1}(\bar{X}, Y) & \hookrightarrow & H_{2 n-1}^{D R}(X) & \longrightarrow & \operatorname{Div} \frac{0}{S / S}(\bar{X}, Y) \otimes k \\
\cong \uparrow & & \cong \uparrow & & \cong \uparrow \\
T_{\mathrm{DR}}\left(\operatorname{Pic}^{0}(\bar{X}, Y)\right) & \hookrightarrow & T_{\mathrm{DR}}\left(\operatorname{Pic}^{-}(X)\right) & \rightarrow & T_{\mathrm{DR}}\left(\operatorname{Div} \frac{0}{S} / S\right.
\end{array}
$$

where: $i)$ the top and bottom rows are obtained by applying $T_{\mathrm{DR}}$ to the earlier diagram of 1-motives, and are exact by construction ( $c f$. Chapter 1), ii) the second row is exact according to $(26)$, iii) the vertical isomorphisms are then obtained by applying Proposition 2.6.1, Lemma 2.6.2 and Corollary 2.6.4, yielding the top row of (26) as the top row of De Rham realizations in (27). 



\section{CHAPTER 3}

\section{COHOMOLOGICAL ALBANESE 1-MOTIVE: $\mathrm{Alb}^{+}$}

We keep the same notations and hypotheses of the previous chapter.

\subsection{Definition of $\mathrm{Alb}^{+}$}

Let $X$ be a variety over an algebraically closed field $k$ of characteristic 0 . To define our cohomological Albanese $\mathrm{Alb}^{+}(X)$, we just take the Cartier dual of $\mathrm{Pic}^{-}(X)$. We are then left with finding a "more explicit" description of $\mathrm{Alb}^{+}$, if possible; this is given by Proposition 3.1.4, when $X$ is smooth, and by (28), when $X$ is proper.

Definition 3.1.1. - For an algebraic variety $X$ over an algebraically closed field $k$ of characteristic zero we define the following 1-motive

$$
\operatorname{Alb}^{+}(X) \stackrel{\text { def }}{=} \operatorname{Pic}^{-}(X)^{\vee}=\left[\operatorname{Div} \frac{0}{S} / S(\bar{X}, Y) \rightarrow \operatorname{Pic}^{0}(\bar{X}, Y)\right]^{\vee} .
$$

We call $\mathrm{Alb}^{+}(X)$ the cohomological Albanese 1-motive of $X$. Since $\mathrm{Pic}^{-}$is independent of the choices of resolutions and compactifications so is $\mathrm{Alb}^{+}$.

We recall that Deligne's definition of "motivic cohomology" of a curve $C$ (see [15], cf. $[\mathbf{1 6}])$ is the 1 -motive

$$
H_{m}^{1}(C)(1) \stackrel{\text { def }}{=}\left[\operatorname{Div}_{F}^{0}\left(\bar{C}^{\prime}\right) \rightarrow \operatorname{Pic}^{0}\left(\bar{C}^{\prime}\right)\right]
$$

where $\bar{C}^{\prime}$ is a compactification of the semi-normalization $C^{\prime}$ of the given curve $C$, such that $F=\bar{C}^{\prime}-C^{\prime}$ is a finite set of non-singular points. We can relate Deligne's definition to ours.

Proposition 3.1.2. - If C is a curve (i.e., a purely 1-dimensional variety) over an algebraically closed field $k$ of characteristic 0, we have a canonical identification

$$
H_{m}^{1}(C)(1) \cong \operatorname{Alb}^{+}(C)
$$


Proof. - The normalization $\widetilde{C}$ of $C$ clearly factors through the semi-normal curve $C^{\prime}$ and the morphism $C^{\prime} \rightarrow C$ is bijective on points, and so induces an isomorphism on the groups of Weil divisors. We therefore have that $\mathrm{Alb}^{+}(C)=\mathrm{Alb}^{+}\left(C^{\prime}\right)$. On the other hand, $H_{m}^{1}(C)(1)=H_{m}^{1}\left(C^{\prime}\right)(1)$ as well, from Deligne's definition.

We then can assume $C=C^{\prime}$ itself to be semi-normal; let $\pi: \widetilde{C} \rightarrow C$ be the normalization. First consider the compact case, i.e., $\bar{C}^{\prime}=C^{\prime}=C$. We then have a canonical quasi-isomorphism

$$
\left[\mathcal{O}_{C}^{*} \rightarrow i_{*} \mathcal{O}_{S}^{*}\right] \cong\left[R \pi_{*} \mathcal{O}_{C}^{*} \rightarrow R \pi_{*} \widetilde{i}_{*} \mathcal{O}_{\widetilde{S}}^{*}\right]
$$

where $i: S \hookrightarrow C$ is the embedding of the finite set $S$ of singular points and $\widetilde{i}: \widetilde{S} \hookrightarrow \widetilde{C}$ the embedding of the inverse image of $S$ : therefore, we get an isomorphism

$$
\operatorname{Pic}(C, S) \cong \operatorname{Pic}(\widetilde{C}, \widetilde{S})
$$

From the exact sequences (5) we get the following diagram

$$
\begin{aligned}
& 0 \\
& 0 \rightarrow \frac{H^{0}\left(S, \mathcal{O}_{S}^{*}\right)}{H^{0}\left(C, \mathcal{O}_{C}^{*}\right)} \rightarrow \operatorname{Pic}^{0}(C, S) \rightarrow \operatorname{Pic}^{0}(C) \rightarrow 0 \\
& 0 \rightarrow \frac{H^{0}\left(\widetilde{S}, \mathcal{O}_{\widetilde{S}}^{*}\right)}{H^{0}\left(\widetilde{C}, \mathcal{O}_{\widetilde{C}}^{*}\right)} \rightarrow \operatorname{Pic}^{0}(\widetilde{C}, \widetilde{S}) \rightarrow \operatorname{Pic}^{0}(\widetilde{C}) \rightarrow 0 \\
& 0 \rightarrow \operatorname{Div}_{\widetilde{S} / S}^{\stackrel{\downarrow}{0}}(\widetilde{C})^{\vee} \rightarrow \operatorname{Pic}^{0}(C) \quad \rightarrow \operatorname{Pic}^{0}(\widetilde{C}) \rightarrow 0
\end{aligned}
$$

showing that $\left[\operatorname{Div} \underset{\widetilde{S} / S}{0}(\widetilde{C}) \rightarrow \operatorname{Pic}^{0}(\widetilde{C})\right]$ is Cartier dual of $\operatorname{Pic}^{0}(C)=H_{m}^{1}(C)(1)(c f$. $[53])$.

If $C$ is not compact, let $\bar{C}$ be a smooth compactification of the normalization $\widetilde{C}$, and set $F=\bar{C}-C$; then $\left[\operatorname{Div}_{F}^{0}(\bar{C}) \rightarrow \operatorname{Pic}^{0}(\bar{C})\right]$ dualizes to

$$
0 \rightarrow \frac{H^{0}\left(F, \mathcal{O}_{F}^{*}\right)}{H^{0}\left(\bar{C}, \mathcal{O}_{\bar{C}}^{*}\right)} \rightarrow \operatorname{Pic}^{0}(\bar{C}, F) \rightarrow \operatorname{Pic}^{0}(\bar{C}) \rightarrow 0
$$

One can then see that the symmetric avatars of $\mathrm{Alb}^{+}(C)$ and $H_{m}^{1}(C)(1)$ are the same, e.g., by making use of the "classical" Lemma 3.1.3.

The proof of the following fact is left as an exercise for the reader.

LEMma 3.1.3. - Let $C$ be a non-singular projective curve. Let $S$ and $T$ be disjoint finite sets of closed points. Then we have the following duality isomorphism between 
1-motives

$$
\left[\operatorname{Div}_{S}^{0}(C) \rightarrow \operatorname{Pic}^{0}(C, T)\right]^{\vee}=\left[\operatorname{Div}_{T}^{0}(C) \rightarrow \operatorname{Pic}^{0}(C, S)\right] .
$$

We have that $\operatorname{Alb}^{+}(X)$ is a semi-abelian variety whenever $X$ is proper over $k$; in fact, in this case $\bar{X}=\widetilde{X}$, i.e., $Y=\varnothing$, and $\operatorname{Alb}^{+}(X)$ is given by the following Cartier dual

$$
\operatorname{Alb}^{+}(X)=\left[\operatorname{Div} \underset{S / S}{0}(\widetilde{X}) \rightarrow \operatorname{Pic}^{0}(\widetilde{X})\right]^{\vee}
$$

Thus, if $X$ is a proper $k$-variety, $\mathrm{Alb}^{+}(X)$ can be represented as an extension

$$
0 \rightarrow T(\widetilde{S} / S) \rightarrow \operatorname{Alb}^{+}(X) \rightarrow \operatorname{Alb}(\widetilde{X}) \rightarrow 0
$$

where the torus $T(\widetilde{S} / S)$ has character group $\operatorname{Div} \underset{\widetilde{S} / S}{0}(\widetilde{X})$ (cf. Chapter 1). Therefore, we can regard $\mathrm{Alb}^{+}(X)$ as a $\mathbb{G}_{m}$-bundle over the abelian variety $\operatorname{Alb}(\tilde{X})$.

If $X$ is a smooth variety over $k=\bar{k}$, we then have that $\widetilde{X}=X$, i.e., $\widetilde{S}=S=\varnothing$, whence $\mathrm{Pic}^{-}(X)$ is a semi-abelian variety, and $\mathrm{Alb}^{+}(X)$ is given by a homomorphism from a lattice to an abelian variety. It is natural to ask what these are, "concretely".

Let $\bar{X}$ be a non-singular proper variety over $k=\bar{k}$, and $Y \subset \bar{X}$ a normal crossing divisor. Denote by $\mathbb{Z}^{Y}$ and $\mathbb{Z}^{\bar{X}}$ the free abelian groups generated by the connected components of $Y$ and $\bar{X}$ respectively (note that the connected components of $\bar{X}$ are irreducible, but this need not hold for $Y$ ). Then there is a canonical homomorphism $\gamma: \mathbb{Z}^{Y} \rightarrow \mathbb{Z}^{\bar{X}}$ induced by the mapping that takes a component of $Y$ to the component of $\bar{X}$ to which it belongs. The kernel of $\gamma$ is generated by classes $\left[Y_{I}\right]-\left[Y_{J}\right]$ where $Y_{I}$ and $Y_{J}$ are distinct connected components of $Y$ contained in the same component of $\bar{X}$.

Let $Y_{I}$ and $Y_{J}$ be distinct connected components of $Y$, contained in the same component of $\bar{X}$, and choose (closed) points $y_{I} \in Y_{I}$ and $y_{J} \in Y_{J}$. Then we consider $a_{\bar{X}}\left(y_{I}-y_{J}\right) \in \operatorname{Alb}(\bar{X})$, where $a_{\bar{X}}: \mathcal{Z}_{0}(\bar{X})_{0} \rightarrow \operatorname{Alb}(\bar{X})$ denotes the Albanese mapping for zero-cycles of degree zero. If $\widetilde{Y} \rightarrow Y$ is the normalization, then $\tilde{Y} \rightarrow \bar{X}$ is a morphism between smooth and proper varieties, and so yields a morphism $\operatorname{Alb}(\widetilde{Y}) \rightarrow$ $\operatorname{Alb}(\bar{X})$ of abelian varieties. Note that $\tilde{Y}=\coprod_{i} Y_{i}$, and $\operatorname{Alb}(\tilde{Y})=\oplus_{i} \operatorname{Alb}\left(Y_{i}\right)$, where $Y=\cup_{i} Y_{i}$ is the decomposition into irreducible components.

Proposition 3.1.4. - Let $\bar{X}$ be a smooth proper $k$-variety, and $Y$ a normal crossing divisor in $\bar{X}$. The Cartier dual of $\operatorname{Pic}^{0}(\bar{X}, Y)$ is the 1 -motive given by the lattice

$$
\mathbb{Z}^{(\bar{X}, Y) \stackrel{\text { def }}{=}} \operatorname{ker}\left(\mathbb{Z}^{Y} \stackrel{\gamma}{\rightarrow} \mathbb{Z}^{\bar{X}}\right)=T(\bar{X}, Y)^{\vee},
$$

the abelian variety

$$
\frac{\operatorname{Alb}(\bar{X})}{\operatorname{im}\left(\oplus_{i} \operatorname{Alb}\left(Y_{i}\right)\right)}=\left(\operatorname{ker}^{0}\left(\operatorname{Pic}^{0}(\bar{X}) \rightarrow \oplus_{i} \operatorname{Pic}^{0}\left(Y_{i}\right)\right)\right)^{\vee}
$$

and the homomorphism of group schemes

$$
u_{X}: \mathbb{Z}^{(\bar{X}, Y)} \rightarrow \frac{\operatorname{Alb}(\bar{X})}{\operatorname{im}\left(\oplus \operatorname{Alb}\left(Y_{i}\right)\right)}
$$


defined by

$$
u_{X}\left(Y_{I}-Y_{J}\right)=a_{\bar{X}}\left(y_{I}-y_{J}\right) \quad\left(\bmod \operatorname{im} \oplus_{i} \operatorname{Alb}\left(Y_{i}\right)\right)
$$

where $Y_{I}, Y_{J}$ lie in the same component of $X$, and $y_{I} \in Y_{I}, y_{J} \in Y_{J}$ are any closed points. Therefore,

$$
\operatorname{Alb}^{+}(X)=\left[\mathbb{Z}^{(\bar{X}, Y)} \stackrel{u_{X}}{\longrightarrow} \frac{\operatorname{Alb}(\bar{X})}{\operatorname{im}\left(\oplus_{i} \operatorname{Alb}\left(Y_{i}\right)\right)}\right] .
$$

Proof. - We first note that the homomorphism $u_{X}$ is well-defined; in fact if $y_{I}^{\prime}, y_{J}^{\prime}$ is another such pair of points, then we easily see that $a_{\bar{X}}\left(y_{I}-y_{J}\right)-a_{\bar{X}}\left(y_{I}^{\prime}-y_{J}^{\prime}\right)$ lies in the image of $\oplus_{i} \operatorname{Alb}\left(Y_{i}\right) \rightarrow \operatorname{Alb}(\bar{X})$ (first we consider the case when the pair of points $y_{I}^{\prime}, y_{I}$, as well as $y_{J}^{\prime}, y_{J}$, each lie in an irreducible component of $Y$; then we can deduce the general case).

By (5) it is clear that the character group of the torus $T(\bar{X}, Y)$ is given by the lattice $\mathbb{Z}^{(\bar{X}, Y)}$. The following pull-back homomorphism between abelian varieties

$$
\operatorname{Pic}^{0}(\bar{X}) \stackrel{\rho}{\longrightarrow} \oplus_{i} \operatorname{Pic}^{0}\left(Y_{i}\right)
$$

is dual to the following push-forward homomorphism

$$
\oplus_{i} \operatorname{Alb}\left(Y_{i}\right) \rightarrow \operatorname{Alb}(\bar{X})
$$

Thus

as claimed.

In order to check that the map $u_{X}$ is Cartier dual to $\operatorname{Pic}^{0}(\bar{X}, Y)$, it suffices to show that $u_{X}$ coincides, on each generator $\left[Y_{I}\right]-\left[Y_{J}\right]$ of $\mathbb{Z}^{(\bar{X}, Y)}$, with the analogous homomorphism for the Cartier dual 1-motive. Choosing points $y_{I} \in Y_{I}, y_{j} \in Y_{J}$ which are smooth on $Y$, one can reduce (by considering the normalization of an irreducible curve passing through the pair of points, and standard functoriality for Picard and Albanese varieties) to checking the duality assertion when $\bar{X}$ is a smooth connected projective curve, and $Y$ consists of 2 points, for which it is "classical" (see [53] for a more general statement; see also [48, Exemple, pg.11-04], and [37]).

We can now show that the Albanese 1-motive $\mathrm{Alb}^{+}$is a birational invariant of normal proper varieties, and that in fact it is given by the Albanese variety of any resolution of singularities of $X$. More generally, we have the following.

Proposition 3.1.5. - If $X$ is a normal $k$-variety the Albanese 1-motive $\mathrm{Alb}^{+}(X)$ is the Cartier dual of $\operatorname{Pic}^{0}(\bar{X}, Y)$. In particular, if $X$ is also proper, then $\operatorname{Alb}^{+}(X)=$ $\operatorname{Alb}(\bar{X})$.

Proof. - First consider the case when $X$ is a proper, normal surface. The proposition is true in this case because the intersection matrix of the exceptional divisor of a desingularization of a normal surface singularity is known [35] to be negative 
definite: the group $\operatorname{Div} \underset{\widetilde{S}}{0}(\widetilde{X})$ is zero since any non-zero linear combination of compact components of $\widetilde{S}$ cannot be numerically equivalent to zero.

For higher dimensional proper $X$, we take $\bar{X}$ to be smooth and projective; now by choosing successive hyperplane sections, we can find a complete intersection smooth surface $T$ in $\widetilde{X}$ and a commutative square

$$
\begin{array}{ccc}
\operatorname{Div}_{\widetilde{S}}(\widetilde{X}) & \rightarrow N S(\widetilde{X}) \\
\downarrow & & \downarrow \\
\operatorname{Div}_{\widetilde{S} \cap T}(\widetilde{T}) & \rightarrow & N S(T)
\end{array}
$$

where $\widetilde{S} \cap T \subset T$ is a reduced normal crossing divisor. Since $T$ is general $\operatorname{Div}_{\widetilde{S}}(\widetilde{X})$ injects into $\operatorname{Div}_{\widetilde{S} \cap T}(T)$. If $T_{0}$ is the normalization of the image of $T$ in $X$, then $T \rightarrow T_{0}$ is a resolution of singularities of a normal proper surface, with exceptional divisor $\widetilde{S} \cap T$; hence $\operatorname{Div} \underset{\widetilde{S} \cap T}{0}(T)=0$ by the case of surfaces considered above, and so $\operatorname{Div} \underset{S}{0}(\widetilde{X})=0$ as well.

If $X$ is open we just notice that $\operatorname{Div} \frac{0}{S}(\bar{X}, Y)$ is contained in $\operatorname{Div} \frac{0}{S}(\bar{X})$; however, the latter group can be assumed to vanish, since $\bar{X}$ can be chosen to be a projective resolution of a normal compactification of $X$.

Remark 3.1.6. - After Proposition 3.1.5, we have the following alternative description of $\mathrm{Pic}^{-}(X)$, for a proper $k$-variety $X$.

Let $X_{n}$ be the normalization of $X, S_{n}$ be the pull-back of the singular locus, and $\widetilde{X}$ a resolution of the normalization. We then have an exact sequence

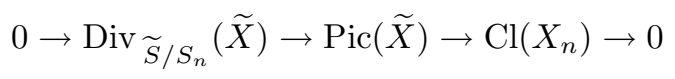

where $\mathrm{Cl}$ denotes the divisor class group and $\operatorname{Div}_{\widetilde{S} / S_{n}}(\widetilde{X})$ is the group generated by exceptional divisors for $\widetilde{X} \rightarrow X_{n}$. Equivalently, $\operatorname{Div}_{\widetilde{S} / S_{n}}(\widetilde{X})$ is the kernel of the push-forward map $\operatorname{Div}(\widetilde{X}) \rightarrow \operatorname{Div}\left(X_{n}\right)$; it is also the kernel of the push-forward map $\operatorname{Div}_{\widetilde{S}}(\widetilde{X}) \rightarrow \operatorname{Div}_{S_{n}}\left(X_{n}\right)$.

We have $\operatorname{Div} \underset{\widetilde{S} / S_{n}}{0}(\widetilde{X})=0$, by Proposition 3.1.5. Hence $\operatorname{Div}_{\widetilde{S} / S_{n}}(\widetilde{X})$ has no intersection with $\operatorname{Pic}^{0}(\widetilde{X})$, and so $\operatorname{Pic}^{0}(\widetilde{X})$ injects into $\mathrm{Cl}\left(X_{n}\right)$; denote its image by $\mathrm{Cl}^{0}\left(X_{n}\right)$. Let Div $S_{n} / S\left(X_{n}\right)$ denote the group of Weil divisors on $X_{n}$ have vanishing push-forward in $X$; these divisors are necessarily supported on $S_{n}$. Let $\operatorname{Div}_{S_{n} / S}^{0}\left(X_{n}\right)$ be the inverse image of $\mathrm{Cl}^{0}\left(X_{n}\right)$ under the obvious map $\operatorname{Div}_{S_{n} / S}\left(X_{n}\right) \rightarrow \mathrm{Cl}\left(X_{n}\right)$ which send a Weil divisor to its divisor class.

We can now define a class group 1-motive of $X$ to be the following 1-motive:

$$
\left[\operatorname{Div}_{S_{n} / S}^{0}\left(X_{n}\right) \rightarrow \mathrm{Cl}^{0}\left(X_{n}\right)\right] .
$$


We then have that the homological Picard 1-motive $\mathrm{Pic}^{-}(X)$ is canonically isomorphic to the class group 1-motive

$$
\operatorname{Pic}^{-}(X) \cong\left[\operatorname{Div}_{S_{n} / S}^{0}\left(X_{n}\right) \rightarrow \mathrm{Cl}^{0}\left(X_{n}\right)\right] .
$$

Note that this description of $\mathrm{Pic}^{-}(X)$ does not involve any resolution of singularities of $X$; in particular, it yields a possible description of $\operatorname{Pic}^{-}(X)$ in positive characteristics as well. We do not pursue this idea further here.

\subsection{Albanese mappings to $\mathrm{Alb}^{+}$}

Let $X$ be an equidimensional proper $k$-variety of dimension $n$, where $k$ is algebraically closed of characteristic 0 . Let $X_{\text {reg }}$ denote the set of smooth points of $X$. We may also consider $X_{\text {reg }}$ as an open subscheme of any given resolution of singularities $\widetilde{X}$. Let $X_{\text {reg }}=\coprod_{j} U_{j}$ be the decomposition into irreducible (or equivalently connected) components. If $\widetilde{X} \rightarrow X$ is a resolution, then the Zariski closures $\overline{U_{j}} \subset \widetilde{X}$ are the irreducible (equivalently, connected) components of $\tilde{X}$.

Choose base points $x_{j} \in U_{j}$ for each $j$, and let $\mathbf{x}=\left\{x_{j}\right\}_{j}$. Let $a_{\mathbf{x}}: \widetilde{X} \rightarrow \operatorname{Alb}(\tilde{X})$ be the corresponding Albanese mapping. Since $X$ is proper over $k, \operatorname{Alb}^{+}(X)$ is a torus bundle over $\operatorname{Alb}(\widetilde{X})$. Consider the following pull-back square

$$
\begin{array}{ll}
\operatorname{Alb}^{+}(X) & \rightarrow \operatorname{Alb}(\widetilde{X}) \\
\widetilde{a}_{\mathbf{x}} \uparrow & \uparrow a_{\mathbf{x}} \\
\operatorname{Alb}^{\dagger}(X) & \rightarrow \widetilde{X}
\end{array}
$$

Then $\operatorname{Alb}^{\dagger}(X)$ is a torus bundle on $\widetilde{X}$, with toric fiber

$$
T(\widetilde{S} / S) \stackrel{\text { def }}{=} \operatorname{Hom}\left(\operatorname{Div} \underset{S}{0} / S(\widetilde{X}), \mathbb{G}_{m}\right)
$$

We claim that the restriction of the torus bundle $\operatorname{Alb}^{\dagger}(X) \rightarrow \widetilde{X}$ to the open subset $X_{\text {reg }} \subset \widetilde{X}$ has a natural trivialization. In fact, dually, any divisor $D$ in $\operatorname{Div} \underset{\widetilde{S} / S}{0}(\widetilde{X})=$ $T(\widetilde{S} / S)^{\vee}$ is mapped to the class in $\operatorname{Pic}^{0}(\widetilde{X})$ of the line bundle $\mathcal{O}(D)$, which is canonically trivialized on $X_{\text {reg }}$, since $\operatorname{supp}(D) \cap X_{\text {reg }}=\varnothing$. Therefore, by a "classical" argument due to Severi $(c f .[48, \S 1])$ there is a section $\sigma: X_{\text {reg }} \rightarrow \operatorname{Alb}^{\dagger}(X)$. By composing $\sigma$ with $\widetilde{a}_{\mathbf{x}}$ we get the Albanese mapping

$$
a_{\mathbf{x}}^{+}: X_{\text {reg }} \rightarrow \mathrm{Alb}^{+}(X) \text {. }
$$

It is easy to see that $a_{\mathbf{x}}^{+}$is independent of the choice of the resolution of singularities $\widetilde{X}$ of $X$.

If $X$ is not equidimensional, let $X^{(n)}$ denote the union of its $n$-dimensional irreducible components. We define $X_{\text {reg }}$ to be the intersection of $X^{(n)}$ with the locus of smooth points of $X$. Since $\operatorname{Alb}^{+}(X)=\operatorname{Alb}^{+}\left(X^{(n)}\right)$, while $X_{\text {reg }} \subset X_{\text {reg }}^{(n)}$, we obtain 
an Albanese mapping $a_{\mathbf{x}}^{+}: X_{\mathrm{reg}} \rightarrow \mathrm{Alb}^{+}(X)$ by restricting that of $X^{(n)}$, if the base points $x_{j}$ are chosen in $X_{\text {reg. }}$.

\subsection{Hodge, étale and De Rham realizations of $\mathrm{Alb}^{+}$}

Let $X$ be an $n$-dimensional variety over $\mathbb{C}$. We recall that Cartier duality for 1-motives is compatible, under the Hodge realization, with the canonical involution $H \longmapsto \operatorname{Hom}(H, \mathbb{Z}(1))$ on the category of mixed Hodge structures. We thus have the following consequence of Theorem 2.4.5.

Corollary 3.3.1. - Let $X$ be as above. Then

$$
T_{\text {Hodge }}\left(\operatorname{Alb}^{+}(X)\right) \cong H^{2 n-1}(X, \mathbb{Z}(n)) /(\text { torsion })
$$

Proof. - We have the formula

$$
\operatorname{Hom}\left(H_{2 n-1}(X, \mathbb{Z}(1-n)), \mathbb{Z}(1)\right)=H^{2 n-1}(X, \mathbb{Z}(n)) /(\text { torsion })
$$

in the category of mixed Hodge structures. Cartier duality for 1-motives and Theorem 2.4.5 then yield the result.

We let

We then have:

$$
J^{n}(X) \stackrel{\text { def }}{=} \frac{H^{2 n-1}(X, \mathbb{C}(n))}{F^{0} H^{2 n-1}(X, \mathbb{C}(n))+\operatorname{im} H^{2 n-1}(X, \mathbb{Z}(n))} .
$$

Corollary 3.3.2. - Let $X$ be a proper variety over $\mathbb{C}$ and $n=\operatorname{dim} X$. The Albanese 1-motive $\operatorname{Alb}^{+}(X)$ is canonically isomorphic to the semi-abelian variety $J^{n}(X)$, given as an algebraic extension

$$
0 \rightarrow T \rightarrow J^{n}(X) \stackrel{f^{*}}{\longrightarrow} J^{n}(\widetilde{X}) \rightarrow 0
$$

where $f: \widetilde{X} \rightarrow X$ is any resolution of singularities, and the torus $T$ is given by

$$
\frac{H^{2 n-2}(\widetilde{S}, \mathbb{Z})}{\operatorname{im}\left(H^{2 n-2}(S, \mathbb{Z}) \oplus H^{2 n-2}(\widetilde{X}, \mathbb{Z})\right)} \otimes \mathbb{C}^{*}
$$

Proof. - This follows from the Mayer-Vietoris sequence of mixed Hodge structures

$$
H^{2 n-2}(S, \mathbb{Z}) \oplus H^{2 n-2}(\widetilde{X}, \mathbb{Z}) \rightarrow H^{2 n-2}(\widetilde{S}, \mathbb{Z}) \rightarrow H^{2 n-1}(X, \mathbb{Z}) \rightarrow H^{2 n-1}(\widetilde{X}, \mathbb{Z})
$$

Tate twisted by $n$, where: $H^{2 n-2}(\widetilde{S}, \mathbb{Z}(n))$ is pure of weight -2 and $H^{2 n-1}(\widetilde{X}, \mathbb{Z}(n))$ is pure of weight -1 .

In fact, the Deligne 1-motive canonically associated to $H^{2 n-1}(X, \mathbb{Z}(n))$ is exactly the claimed semi-abelian variety $J^{n}(X)$ but, by the Theorem 2.4.5, the Hodge realization of $\mathrm{Alb}^{+}(X)$ is also $H^{2 n-1}(X, \mathbb{Z}(n))$, modulo torsion, and the Hodge realization functor is fully faithful.

We now let $X$ be a variety over an algebraically closed field $k$ of characteristic zero. 
Corollary 3.3.3. - Let $X$ be as above and $n=\operatorname{dim} X$. Then

$$
\widehat{T}\left(\operatorname{Alb}^{+}(X)\right) \cong H_{e ́ t}^{2 n-1}(X, \widehat{\mathbb{Z}}(n)) /(\text { torsion })
$$

Proof. - This follows from the formula

$$
\operatorname{Hom}\left(H_{2 n-1}^{\text {ét }}(X, \widehat{\mathbb{Z}}(1-n)), \widehat{\mathbb{Z}}(1)\right)=H_{\text {êt }}^{2 n-1}(X, \widehat{\mathbb{Z}}(n)) /(\text { torsion })
$$

and Theorem 2.5.4.

Let $X$ be any $n$-dimensional variety over an algebraically closed field of characteristic zero as above. Recall $(2.6 .3)$ that $H_{\mathrm{DR}}^{*}(X) \stackrel{\text { def }}{=} \mathbb{H}^{*}\left(\bar{X}_{\bullet}, \Omega_{\bar{X}}\left(\log \left(Y_{\bullet}\right)\right)\right)$, the De Rham cohomology (filtered) $k$-vector spaces of $X$, where $\bar{X}$. is any smooth compactification of a proper smooth hypercovering $X$. with normal crossing boundary $Y$.

Corollary 3.3.4. - Let $X$ be as above. Then

$$
T_{\mathrm{DR}}\left(\operatorname{Alb}^{+}(X)\right) \cong H_{\mathrm{DR}}^{2 n-1}(X)(n)
$$

Proof. - By Theorem 2.6.6, as above, we get the result. 


\section{CHAPTER 4}

\section{COHOMOLOGICAL PICARD 1-MOTIVE: Pic $^{+}$}

\subsection{Simplicial Picard functor}

We first extend some results from the folklore on Picard functors to the context of simplicial schemes. Presumably these are known to experts, though we do not have any reference for these facts.

Let $\pi: V . \rightarrow S$ be a simplicial scheme over a base scheme $S$. We will denote by $\operatorname{Pic}(V$.$) the group of isomorphism classes of simplicial line bundles on V$. (i.e., of invertible $\mathcal{O}_{V_{\bullet}}$-modules). We have the following description of $\operatorname{Pic}\left(V_{\bullet}\right)$. Denote by $d_{k}^{i}: V_{i} \rightarrow V_{i-1}$ the faces map of the given simplicial scheme $V$. , and consider the following set of data and conditions:

- a line bundle : $\mathcal{L}$ on $V_{0}$;

- an isomorphism : $\alpha:\left(d_{0}^{1}\right)^{*}(\mathcal{L}) \stackrel{\cong}{\cong}\left(d_{1}^{1}\right)^{*}(\mathcal{L})$ on $V_{1}$;

satisfying the

- cocycle condition : that the following composite

$$
\left(\left(d_{1}^{2}\right)^{*}(\alpha)\right)^{-1} \circ\left(\left(d_{2}^{2}\right)^{*}(\alpha)\right) \circ\left(\left(d_{0}^{2}\right)^{*}(\alpha)\right)
$$

yields $1 \in \Gamma\left(V_{2}, \mathbb{G}_{m}\right)$, i.e., if we let

$$
\begin{aligned}
& f_{0} \stackrel{\text { def }}{=} d_{0}^{1} d_{0}^{2}=d_{0}^{1} d_{1}^{2} \\
& f_{1} \stackrel{\text { def }}{=} d_{0}^{1} d_{2}^{2}=d_{1}^{1} d_{0}^{2} \\
& f_{2} \stackrel{\text { def }}{=} d_{1}^{1} d_{2}^{2}=d_{1}^{1} d_{1}^{2}
\end{aligned}
$$

then we want that the following diagram

$$
\left(d_{1}^{2}\right)^{*}(\alpha) f_{\substack{f_{0}^{*}(\mathcal{L}) \\ \swarrow}} f_{1}^{*}(\mathcal{L})
$$

commutes 
We clearly then have the following.

Proposition 4.1.1. - Let V. be a simplicial scheme. Elements of $\mathbf{P i c}(V \cdot)$ correspond to isomorphism classes of pairs $(\mathcal{L}, \alpha)$ as above, satisfying the cocycle condition. Moreover, there is a functorial isomorphism

$$
\operatorname{Pic}\left(V_{\bullet}\right) \cong \mathbb{H}^{1}\left(V_{\bullet}, \mathcal{O}_{V_{\bullet}}^{*}\right) .
$$

Proof. - The identification of $\mathbf{P i c}(V \cdot)$ with isomorphism classes of pairs $(\mathcal{L}, \alpha)$ is easy, and left to the reader. For a proof of the cohomological description, see Appendix .

We now consider the simplicial Picard functor on the category of schemes over $S$, which we denote as follows

$$
T \longmapsto \mathbf{P i c}_{V \cdot / S}(T)
$$

obtained by sheafifying the functor

$$
T \longmapsto \operatorname{Pic}\left(V \cdot \times_{S} T\right)
$$

with respect to the fpqc-topology. This means that if $\pi: V \cdot \times_{S} T \rightarrow T$, then

$$
\operatorname{Pic}_{V \cdot / S}(T) \cong H_{f p q c}^{0}\left(T, R^{1} \pi_{*}\left(\mathcal{O}_{V \cdot \times_{S} T}^{*}\right)\right) \text {. }
$$

As usual, if $\pi_{*}\left(\mathcal{O}_{V \cdot}^{*}\right)=\mathcal{O}_{S}^{*}$, the Leray spectral sequence along $\pi$ and descent yields an exact sequence

$$
0 \rightarrow \operatorname{Pic}(S) \rightarrow \operatorname{Pic}\left(V_{\bullet}\right) \rightarrow \mathbf{P i c}_{V_{\bullet} / S}(S) \rightarrow H^{2}\left(S, \mathbb{G}_{m}\right) \rightarrow \mathbb{H}^{2}\left(V_{\bullet}, \mathcal{O}_{V_{\bullet}}^{*}\right) .
$$

Furthermore, if there is a section of $\pi$, we have that

$$
\operatorname{Pic}_{V . / S}(S) \cong \frac{\operatorname{Pic}(V \cdot)}{\operatorname{Pic}(S)} \text {. }
$$

We are mainly interested in the case when $S$ is the spectrum of a field $k$ and $X$. is a proper (smooth) simplicial scheme over $k$; the previous description for $k$-points of $\mathbf{P i c}_{X . / k}$ (i.e., the formula $\left.\mathbf{P i c}_{V . / k}(k) \cong \mathbf{P i c}(V).\right)$ applies in the geometric case (i.e., when $k$ is algebraically closed), since $H^{i}\left(k, \mathbb{G}_{m}\right)=0$ for $i=1,2$ in that case; here, we do not need the assumption that $\pi_{*} \mathcal{O}_{X}$. $=k$.

In order to give another description of the simplicial Picard functor, which is more suitable for our purposes, we consider the canonical spectral sequence

$$
E_{1}^{p, q}=H^{q}\left(X_{p}, \mathcal{O}_{X_{p}}^{*}\right) \Rightarrow \mathbb{H}^{p+q}\left(X_{.}, \mathcal{O}_{X .}^{*}\right)
$$

Let $\pi_{i}: X_{i} \rightarrow k$ denote the structure morphisms. The spectral sequence yields the following exact sequence of $f p q c$-sheaves:

(31) $0 \rightarrow \frac{\operatorname{ker}\left(\left(\pi_{1}\right)_{*} \mathbb{G}_{m, X_{1}} \rightarrow\left(\pi_{2}\right)_{*} \mathbb{G}_{m, X_{2}}\right)}{\operatorname{im}\left(\left(\pi_{0}\right)_{*} \mathbb{G}_{m, X_{0}} \rightarrow\left(\pi_{1}\right)_{*} \mathbb{G}_{m, X_{1}}\right)} \rightarrow \mathbf{P i c}_{X . / k} \rightarrow \operatorname{ker}\left(\operatorname{Pic}_{X_{0} / k} \rightarrow \operatorname{Pic}_{X_{1} / k}\right)$

We have the following facts. 
LEMMA 4.1.2. - If $X$. is smooth and proper over a field $k$, then the simplicial Picard functor $\mathbf{P i c}_{X . / k}$ is representable by a group scheme locally of finite type over $k$.

Proof. - See Appendix .

For smooth proper simplicial schemes over $k=\bar{k}$ we have the following description.

Proposition 4.1.3. - Let $X$. be smooth and proper over $k=\bar{k}$ of characteristic zero. The sequence (31) yields a semi-abelian group scheme over $k$, which can be represented as an extension

$$
1 \rightarrow T(X .) \rightarrow \mathbf{P i c}^{0}(X .) \rightarrow A(X .) \rightarrow 0
$$

where:

(i) $\mathbf{P i c}^{0}(X$.$) is the connected component of the identity of \mathbf{P i c} \mathbf{c}_{X k}$;

(ii) $T(X$.$) is the k$-torus defined by

$$
T(X .) \stackrel{\text { def }}{=} \frac{\operatorname{ker}\left(\left(\pi_{1}\right)_{*} \mathbb{G}_{m, X_{1}} \rightarrow\left(\pi_{2}\right)_{*} \mathbb{G}_{m, X_{2}}\right)}{\operatorname{im}\left(\left(\pi_{0}\right)_{*} \mathbb{G}_{m, X_{0}} \rightarrow\left(\pi_{1}\right)_{*} \mathbb{G}_{m, X_{1}}\right)}
$$

where $\pi_{i}: X_{i} \rightarrow k$ are the structure morphisms;

(iii) $A(X$.$) is the following abelian variety$

$$
A(X .) \stackrel{\text { def }}{=} \operatorname{ker}^{0}\left(\operatorname{Pic}^{0}\left(X_{0}\right) \rightarrow \operatorname{Pic}^{0}\left(X_{1}\right)\right)
$$

obtained as the connected component of the identity of the kernel.

Proof. - From Lemma 4.1.2, by taking connected components of the identity of the group schemes in $(31)$, where $T(X$.$) is connected, we claim that \mathbf{P i c}^{0}(\bar{X}$.) surjects onto the abelian variety $\operatorname{ker}^{0}\left(\mathrm{Pic}_{X_{0} / k}^{0} \rightarrow \operatorname{Pic}_{X_{1} / k}^{0}\right)$ : by the spectral sequence (30), the image of $\operatorname{Pic}(\bar{X}$.$) is the kernel of the following edge homomorphism$

$$
\operatorname{ker}\left(\mathrm{Pic}_{X_{0} / k} \rightarrow \mathrm{Pic}_{X_{1} / k}\right) \rightarrow \frac{\operatorname{ker}\left(\left(\pi_{2}\right)_{*} \mathbb{G}_{m, X_{2}} \rightarrow\left(\pi_{3}\right)_{*} \mathbb{G}_{m, X_{3}}\right)}{\operatorname{im}\left(\left(\pi_{1}\right)_{*} \mathbb{G}_{m, X_{1}} \rightarrow\left(\pi_{2}\right)_{*} \mathbb{G}_{m, X_{2}}\right)}
$$

which vanishes on the connected component of the identity of the domain.

\subsection{Definition of $\mathrm{Pic}^{+}$}

Now let $X$. be a smooth simplicial $k$-variety, which we assume to be obtained from a simplicial pair $\left(\bar{X} ., Y_{\bullet}\right)$ such that $X .=\bar{X} .-Y_{\bullet}, \bar{X}$. is a proper smooth simplicial scheme and $Y$. has components $Y_{i}$ which are normal crossing divisors in $\bar{X}_{i}$. We then have a spectral sequence

$$
E_{1}^{p, q}=H_{Y_{p}}^{q}\left(\bar{X}_{p}, \mathcal{O}_{\bar{X}_{p}}^{*}\right) \Rightarrow \mathbb{H}_{Y .}^{p+q}\left(\bar{X} ., \mathcal{O}_{\bar{X}}^{*}\right) .
$$

Since each component of $\bar{X}$. is smooth we have that $H_{Y_{p}}^{q}\left(\bar{X}_{p}, \mathcal{O}_{\bar{X}_{p}}^{*}\right) \neq 0$ if and only if $q=1$ and we clearly have that

$$
H_{Y_{p}}^{1}\left(\bar{X}_{p}, \mathcal{O}_{\bar{X}_{p}}^{*}\right) \cong \operatorname{Div}_{Y_{p}}\left(\bar{X}_{p}\right) .
$$


From the above spectral sequence we then have

$$
\mathbb{H}_{Y}^{1}\left(\bar{X} ., \mathcal{O}_{\bar{X}}^{*}\right) \cong \operatorname{ker}\left(\operatorname{Div}_{Y_{0}}\left(\bar{X}_{0}\right) \stackrel{d_{0}^{*}-d_{1}^{*}}{\longrightarrow} \operatorname{Div}_{Y_{1}}\left(\bar{X}_{1}\right)\right)
$$

We will denote by $\operatorname{Div}_{Y}(\bar{X}$. $)$ the subgroup of divisors on $\bar{X}_{0}$ given by the right side of (33). The canonical mapping

$$
\operatorname{Div}_{Y_{\bullet}}\left(\bar{X}_{\bullet}\right)=\mathbb{H}_{Y_{\bullet}}^{1}\left(\bar{X}_{\bullet}, \mathcal{O}_{\bar{X}}^{*}\right) \rightarrow \mathbb{H}^{1}\left(\bar{X}_{\bullet}, \mathcal{O}_{\bar{X}}^{*}\right) \cong \mathbf{P i c}_{\bar{X}, / k}(k)
$$

is compatible with the restriction of the map taking a divisor on $\bar{X}_{0}$ to the associated line bundle.

In order to define $\mathrm{Pic}^{+}$, we let

$$
\operatorname{Pic}^{0}(\bar{X} \cdot) \stackrel{\text { def }}{=} \mathbf{P i c}_{\bar{X}_{.} / k}^{0}(k) \subset \mathbf{P i c}(\bar{X} .),
$$

and let $\operatorname{Div}_{Y_{\bullet}}^{0}\left(\bar{X}_{\bullet}\right)$ denote the inverse image of $\operatorname{Pic}^{0}(\bar{X} \cdot)$ under the above mapping (34).

Now let $X$ be an algebraic variety over a field $k=\bar{k}$ of characteristic zero. Let $\pi: X . \rightarrow X$ be a smooth proper hypercovering of $X$, such that we can choose a simplicial pair $\left(\bar{X} ., Y_{\bullet}\right)$ as above (i.e., so that $\bar{X} .-Y .=X$. and $Y$. has normal crossings.)

Definition 4.2.1. - With the hypothesis and notation as above we define the 1motive

$$
\operatorname{Pic}^{+}(X) \stackrel{\text { def }}{=}\left[\operatorname{Div}_{Y \cdot}^{0}(\bar{X} \cdot) \rightarrow \operatorname{Pic}^{0}(\bar{X} \cdot)\right] .
$$

We call $\mathrm{Pic}^{+}(X)$ the cohomological Picard 1-motive of $X$.

Remark 4.2.2. - If $X$ is smooth, let $\bar{X}$ be a smooth compactification with normal crossing boundary $Y$. We may take $\bar{X}$. to be the constant simplicial scheme associated to $\bar{X}$. Then we see easily that $\operatorname{Pic}^{+}(X) \cong\left[\operatorname{Div}_{Y}^{0}(\bar{X}) \rightarrow \operatorname{Pic}^{0}(\bar{X})\right]$.

On the other hand, if $X$ is proper over $k$, then $\bar{X}$. $=X$., and $\operatorname{Pic}^{+}(X)=\operatorname{Pic}^{0}(X$. is a semi-abelian variety.

\subsection{Hodge realization of $\mathrm{Pic}^{+}$}

Let $(\bar{X} ., Y$.$) be a simplicial pair as above. For k=\mathbb{C}$, by the simplicial exponential sequence on $(\bar{X} \cdot)_{\text {an }}$ and GAGA, we have an isomorphism

$$
\left.\operatorname{Pic}(\bar{X} .)=\mathbb{H}^{1}\left(\bar{X} ., \mathcal{O}_{\bar{X}}^{*}\right) \cong \mathbb{H}^{1}\left((\bar{X} \cdot)_{\text {an }}, \mathcal{O}_{(\bar{X}}^{*}\right)_{\text {an }}\right)
$$

and a simplicial cycle map

$$
c \ell .: \operatorname{Pic}(\bar{X} .) \rightarrow \mathbb{H}^{2}(\bar{X} ., \mathbb{Z}(1)) .
$$


Lemma 4.3.1. - Let $\bar{X}$. be as above and $k=\mathbb{C}$. Then

$$
\mathbf{J}^{1}(\bar{X} .) \stackrel{\text { def }}{=} \frac{\mathbb{H}^{1}(\bar{X} \cdot, \mathbb{C}(1))}{F^{0}+\mathbb{H}^{1}(\bar{X} \cdot, \mathbb{Z}(1))} \cong \mathbf{P i c}^{0}(\bar{X} .)
$$

and

$$
\operatorname{Div}_{Y \cdot}^{0}(\bar{X} .) \cong \operatorname{ker}\left(\mathbb{H}_{Y \cdot}^{2}(\bar{X} ., \mathbb{Z}(1)) \rightarrow \mathbb{H}^{2}(\bar{X} ., \mathbb{Z}(1))\right)
$$

Under these isomorphisms the canonical mapping $\operatorname{Div}_{Y \cdot}^{0}\left(\bar{X}_{\bullet}\right) \rightarrow \mathbf{P i c}^{0}(\bar{X} \cdot)$ defined above is identified with an appropriate extension class map for mixed Hodge structures on $\mathbb{H}^{1}(X ., \mathbb{Z}(1))$.

Proof. — From the simplicial exponential sequence, since the complex $\mathbb{Z}(1) . \rightarrow \mathcal{O}_{\bar{X}}$. is quasi-isomorphic to $\mathcal{O}_{\bar{X}}^{*}[-1]$ on $(\bar{X} \cdot)_{\text {an }}$, we have that

$$
\mathbf{J}^{1}(\bar{X} \cdot) \cong \operatorname{ker} c
$$

because

$$
\mathbb{H}^{1}\left(\bar{X} ., \mathcal{O}_{\bar{X}}\right) \cong \frac{\mathbb{H}^{1}(\bar{X} \cdot, \mathbb{C}(1))}{F^{0}}
$$

Since we have a spectral sequence

$$
E_{1}^{p, q}=H_{Y_{p}}^{q}\left(\bar{X}_{p}, \mathbb{Z}(1)\right) \Rightarrow \mathbb{H}_{Y}^{p+q}(\bar{X} ., \mathbb{Z}(1))
$$

such that $E_{1}^{p, *}=0$ for $q=0,1$, we obtain $\mathbb{H}_{Y \cdot}^{1}(\bar{X} ., \mathbb{Z}(1))=0$, and moreover

$$
\mathbb{H}_{Y .}^{2}(\bar{X} \cdot, \mathbb{Z}(1)) \cong \operatorname{ker}\left(H_{Y_{0}}^{2}\left(X_{0}, \mathbb{Z}(1)\right) \stackrel{d_{0}^{*}-d_{1}^{*}}{\longrightarrow} H_{Y_{1}}^{2}\left(X_{1}, \mathbb{Z}(1)\right)\right)
$$

whence $\mathbb{H}_{Y_{\bullet}}^{2}(\bar{X} ., \mathbb{Z}(1)) \cong \operatorname{Div}_{Y_{\bullet}}(\bar{X}$.). The following diagram (with exact bottom row)

$$
\begin{gathered}
\operatorname{Div}_{Y \cdot}^{0}(\bar{X} \cdot) \hookrightarrow \mathbb{H}_{Y \cdot}^{1}\left(\bar{X} \cdot, \mathcal{O}_{\bar{X}}^{*}\right) \stackrel{\cong}{\leftrightarrows} \mathbb{H}_{Y \cdot}^{2}(\bar{X} \cdot, \mathbb{Z}(1)) \\
\downarrow \\
\downarrow \\
\operatorname{Pic}^{0}(\bar{X} \cdot) \hookrightarrow \operatorname{Pic}(\bar{X} \cdot) \stackrel{c \ell}{\longrightarrow} \mathbb{H}^{2}(\bar{X} \cdot, \mathbb{Z}(1))
\end{gathered}
$$

commutes, showing the claimed description of $\operatorname{Div}_{Y \cdot}^{0}(\bar{X} \cdot)$ (note that $\mathbb{H}_{Y}^{1}\left(\bar{X} ., \mathcal{O}_{\bar{X}}^{*}\right.$ ) is computed using the Zariski topology). 
To show that the cycle class coincides with the extension class for the mixed Hodge structure on $\mathbb{H}^{1}(X, \mathbb{Z}(1))$, we consider the following commutative diagram of cohomology groups having exact rows and columns

$$
\begin{aligned}
& 0 \\
& \downarrow \\
& \operatorname{Pic}^{0}(\bar{X} \cdot) \\
& \downarrow \\
& \mathbb{H}_{Y}^{1}\left(\bar{X}_{.}, \mathcal{O}_{\bar{X}}^{*}\right) \rightarrow \mathbb{H}^{1}\left(\bar{X}_{\bullet}, \mathcal{O}_{\bar{X}}^{*}\right) \\
& \mathbb{H}^{1}(\bar{X} \cdot \mathbb{Z}(1)) \quad \rightarrow \quad \mathbb{H}^{1}(X ., \mathbb{Z}(1)) \quad \rightarrow \quad \mathbb{H}_{Y}^{2}\left(\overline{X_{\bullet}}, \mathbb{Z}(1)\right) \quad \rightarrow \mathbb{H}^{2}(\bar{X} \cdot, \mathbb{Z}(1))
\end{aligned}
$$

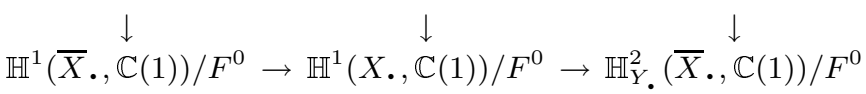

$$
\begin{aligned}
& \begin{array}{c}
\mathbf{J}^{1}(\bar{X} \cdot) \\
\downarrow \\
0
\end{array}
\end{aligned}
$$

The result then follows from a diagram chase ( $c f$. the proof of Lemma 2.4.1 and [9, Lemma 2.8]).

TheOREM 4.3.2. - Let $X$ be defined over $\mathbb{C}$. Then

$$
T_{\text {Hodge }}\left(\operatorname{Pic}^{+}(X)\right) \cong H^{1}(X, \mathbb{Z}(1)) \text {. }
$$

Proof. - We have an exact sequence of mixed Hodge structures

$$
0 \rightarrow \mathbb{H}^{1}(\bar{X} ., \mathbb{Z}(1)) \rightarrow H^{1}(X, \mathbb{Z}(1)) \rightarrow \operatorname{Div}_{Y \cdot}^{0}(\bar{X} \cdot) \rightarrow 0
$$

where $H^{1}(X, \mathbb{Z}(1)) \cong \mathbb{H}^{1}(X ., \mathbb{Z}(1)$. ) by universal cohomological descent: the claim then follows from the Lemma 4.3.1.

\section{4. Étale realization of $\mathrm{Pic}^{+}$}

Let $V$. be any simplicial $k$-scheme. We first need to recall the existence of the following long exact sequence

$$
\cdots \rightarrow \mathbb{H}_{\text {ét }}^{0}\left(V \cdot, \mathbb{G}_{m}\right) \rightarrow \mathbb{H}_{\text {ét }}^{1}\left(V \cdot, \mu_{m}\right) \rightarrow \mathbb{H}_{\text {ét }}^{1}\left(V \cdot, \mathbb{G}_{m}\right) \stackrel{m}{\longrightarrow} \mathbb{H}_{\text {ét }}^{1}\left(V \cdot, \mathbb{G}_{m}\right) \rightarrow \cdots
$$

and a "simplicial Hilbert's Theorem 90".

Proposition 4.4.1. - There is an isomorphism

$$
\mathbb{H}_{e ́ t}^{1}\left(V ., \mathbb{G}_{m}\right) \cong \mathbf{P i c}(V .)
$$

Proof. - Consider the Leray spectral sequence along $\varepsilon_{\bullet}:\left(V_{\bullet}\right)_{\text {ét }} \rightarrow(V \cdot)_{\text {Zar }}$. Since $(\varepsilon \cdot)_{*}\left(\mathbb{G}_{m}\right)=\mathbb{G}_{m}$ we then have a canonical functorial map

$$
\varepsilon_{.}^{*}: \mathbb{H}^{1}\left(V ., \mathcal{O}_{V \cdot}^{*}\right) \rightarrow \mathbb{H}_{\text {ét }}^{1}\left(V ., \mathbb{G}_{m}\right) .
$$


Consider the canonical spectral sequence

$$
E_{1}^{p, q}=H_{\text {êt }}^{q}\left(V_{p}, \mathbb{G}_{m}\right) \Rightarrow \mathbb{H}_{\text {èt }}^{p+q}\left(V, \mathbb{G}_{m}\right)
$$

A similar spectral sequence is clearly available for Zariski cohomology groups, and $\varepsilon^{*}$. is compatible with a morphism between the respective spectral sequences.

Since we have that $H_{\text {ét }}^{q}\left(V_{p}, \mathbb{G}_{m}\right)=H_{\mathrm{Zar}}^{q}\left(V_{p}, \mathcal{O}_{V_{p}}^{*}\right)$ for all $p \geqslant 0$ and $q=0,1$, via $\varepsilon_{p}:\left(V_{p}\right)_{\text {ét }} \rightarrow\left(V_{p}\right)_{\text {Zar }}$, we then get that $\varepsilon^{*}$. is an isomorphism.

We then can see that the cohomology group $\mathbb{H}_{\text {ét }}^{1}\left(V \cdot, \mu_{m}\right)$ is isomorphic to the group given by the isomorphism classes of pairs $(\mathcal{L} ., \eta$. $)$ where $\mathcal{L}$. is a simplicial line bundle and $\eta$. is an isomorphism $\eta$. : $\mathcal{L}^{\otimes m} \cong \mathcal{O}_{V}$. Moreover, we get the following.

Proposition 4.4.2. - We have the "simplicial Kummer sequence"

$$
0 \rightarrow \mathbb{H}^{0}\left(V_{\bullet}, \mathcal{O}_{V_{\bullet}}^{*}\right) / m \stackrel{u}{\longrightarrow} \mathbb{H}_{e t t}^{1}\left(V_{\bullet}, \mu_{m}\right) \stackrel{p}{\longrightarrow} \mathbf{P i c}\left(V_{\bullet}\right)_{m-\text { tors }} \rightarrow 0
$$

where:

- $\mathbb{H}_{e t}^{1}\left(V \cdot, \mu_{m}\right)$ can be regarded as the group of isomorphism classes of triples $(\mathcal{L}, \alpha, \eta)$ given by a line bundle $\mathcal{L}$ on $V_{0}$, an isomorphism $\alpha:\left(d_{0}\right)^{*}(\mathcal{L}) \cong$ $\left(d_{1}\right)^{*}(\mathcal{L})$ on $V_{1}$ satisfying the cocycle condition, an isomorphism $\eta: \mathcal{O}_{V_{0}} \stackrel{\cong}{\longrightarrow} \mathcal{L}^{\otimes m}$ on $V_{0}$ which is compatible with $\alpha^{\otimes m}$ on $V_{1}$, i.e., such that the composite of the following isomorphisms

$$
\begin{aligned}
\mathcal{O}_{V_{1}}=\left(d_{0}\right)^{*}\left(\mathcal{O}_{V_{0}}\right) \stackrel{\left(d_{0}\right)^{*}(\eta)}{\longrightarrow}\left(d_{0}\right)^{*}\left(\mathcal{L}^{\otimes m}\right) \stackrel{\alpha^{\otimes m}}{\longrightarrow} & \left(d_{1}\right)^{*}\left(\mathcal{L}^{\otimes m}\right) \\
& \stackrel{\left(d_{1}\right)^{*}(\eta)^{-1}}{\longrightarrow}\left(d_{1}\right)^{*}\left(\mathcal{O}_{V_{0}}\right)=\mathcal{O}_{V_{1}}
\end{aligned}
$$

is the identity on $\mathcal{O}_{V_{1}}$ (here $d_{0}$ and $d_{1}$ from $V_{1}$ to $V_{0}$ are the face maps of the simplicial scheme);

- $\mathbb{H}^{0}\left(V ., \mathcal{O}_{V_{.}}^{*}\right)$ is given by those units $u_{0} \in H^{0}\left(V_{0}, \mathcal{O}_{V_{0}}^{*}\right)$ such that $d_{0}^{*}\left(u_{0}\right)=d_{1}^{*}\left(u_{0}\right)$ on $V_{1}$;

- the map $u$ is defined by taking a unit $u_{0}$ to the triple $\left(\mathcal{O}_{V_{0}}, 1, u_{0}^{-1}\right)$;

- the map $p$ is defined by taking a triple $(\mathcal{L}, \alpha, \eta)$ to a the torsion pair $(\mathcal{L}, \alpha)$ in the simplicial Picard group.

Proof. - Taking into account Propositions 4.1.1 and 4.4.1, the proof is an easy modification of [33, III.4].

Now let $X$ be a $k$-variety, where $k$ is algebraically closed of characteristic 0 . Fix a smooth proper hypercovering $X$. and a smooth compactification $\bar{X}$. with normal crossing boundary $Y$. For $(D, \mathcal{L} \cdot) \in \operatorname{Div}_{Y \cdot}^{0}\left(\bar{X}_{\bullet}\right) \times \operatorname{Pic}^{0}(\bar{X} \cdot)$ as above, by definition

$$
T_{\mathbb{Z} / m}\left(\operatorname{Pic}^{+}(X)\right)=\frac{\left\{(D, \mathcal{L} \cdot) \mid \eta \cdot: \mathcal{L}_{\cdot}^{\otimes m} \cong \mathcal{O}_{\bar{X} \cdot}(-D)\right\}}{\left\{\left(m D, \mathcal{O}_{\bar{X} \cdot}(-D)\right)\right\}} .
$$


We have a canonical map

$$
\rho_{m}: T_{\mathbb{Z} / m}\left(\operatorname{Pic}^{+}(X)\right) \rightarrow \mathbb{H}_{\text {êt }}^{1}\left(X ., \mu_{m}\right)
$$

defined as follows

$$
\rho_{m}(D, \mathcal{L} .) \stackrel{\text { def }}{=}(\mathcal{L} ., \eta \cdot)_{\mid X} .
$$

Note that $\eta$. is well-defined up to multiplication by an element of $k^{*}$, which is $m$ divisible, so that the isomorphism class of $(\mathcal{L} ., \eta \bullet)_{\mid X}$. is well-defined.

We let

$$
\mathbb{H}_{\text {êt }}^{*}(X ., \widehat{\mathbb{Z}}(1)) \stackrel{\text { def }}{=} \underbrace{\lim }_{m} \mathbb{H}_{\text {ét }}^{*}\left(X ., \mu_{m}\right)
$$

We can show the following.

TheOREM 4.4.3. - Let $X$ be defined over $k$ which is algebraically closed of characteristic zero. Then

$$
\widehat{T}\left(\operatorname{Pic}^{+}(X)\right) \cong H_{e ́ t}^{1}(X, \widehat{\mathbb{Z}}(1)) .
$$

Proof. - If we let $\operatorname{Pic}^{+}(X)$ be given by the 1 -motive $\left[\operatorname{Div}_{Y_{\bullet}}^{0}(\bar{X}.) \rightarrow \operatorname{Pic}^{0}(\bar{X}).\right]$ for a chosen hypercovering and compactification with normal crossing boundary, we get the following commutative diagram (with short exact rows)

$$
\begin{aligned}
& \widehat{T}\left(\operatorname{Pic}^{0}(\bar{X} \cdot)\right) \hookrightarrow \widehat{T}\left(\operatorname{Pic}^{+}(X)\right) \rightarrow \quad \widehat{T}\left(\operatorname{Div}_{Y_{\bullet}}^{0}(\bar{X} \cdot)[1]\right)
\end{aligned}
$$

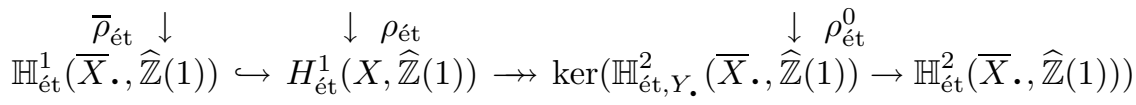

where (i) the bottom row is just the exact sequence of cohomology with supports, (ii) we have

$$
\mathbb{H}_{\text {ét }}^{1}(X . \widehat{\mathbb{Z}}(1)) \cong H_{\text {ét }}^{1}(X, \widehat{\mathbb{Z}}(1)),
$$

since $X . \rightarrow X$ is a universal cohomological descent morphism, and (iii) the top exact sequence is given by (3) in Chapter 1 . We get the mapping $\rho_{\text {ét }}$ above by taking the inverse limit of $\rho_{m} ; \bar{\rho}_{\text {ét }}$ is the induced map. From the above description the mapping $\rho_{\text {êt }}^{0}$ is an isomorphism: in fact, it is easy to see that we have an isomorphism $\widehat{T}\left(\operatorname{Div}_{Y_{\bullet}}(\bar{X} \cdot)\right) \cong \mathbb{H}_{\text {ét, } Y_{\bullet}}^{2}(\bar{X} ., \widehat{\mathbb{Z}}(1))$ such that the following diagram

$$
\begin{gathered}
\widehat{T}\left(\operatorname{Div}_{Y \cdot}(\bar{X} \cdot)\right) \stackrel{\cong}{\rightrightarrows} \mathbb{H}_{\text {ét, }, Y}^{2}(\bar{X} \cdot, \mathbb{Z}(1)) \\
\downarrow \\
\widehat{\operatorname{Pic}(\bar{X}} \cdot) \stackrel{\widehat{c \ell}}{\longrightarrow} \mathbb{H}_{\text {ét }}^{2}(\bar{X} ., \widehat{\mathbb{Z}}(1))
\end{gathered}
$$

commutes (here $\widehat{T}\left(\operatorname{Div}_{Y_{\bullet}}(\bar{X} \cdot)\right)$ and $\left.\widehat{\operatorname{Pic}(\bar{X}} \cdot\right)$ are the profinite completions of the lattice $\operatorname{Div}_{Y_{.}}(\bar{X} \cdot)$ and $\operatorname{Pic}(\bar{X} \cdot)$, respectively).

Granting this, we are left to show that $\bar{\rho}_{\text {ét }}$ is an isomorphism. The latter follows from the fact that the Néron-Severi group of such a smooth proper simplicial $k$-scheme $\bar{X}$. (i.e., the group of connected components of $\operatorname{Pic}(\bar{X}$.$) ) is finitely$ 
generated, whence $\widehat{T}\left(\mathbf{P i c}^{0}(\bar{X} \cdot)\right)=\varliminf_{m} \mathbf{P i c}(\bar{X} \cdot)_{m-\text { tors }}$ and, by the simplicial variants of Hilbert's Theorem 90 and Kummer theory (see (4.4.1) and (4.4.2)), we have $\operatorname{Pic}(\bar{X} \cdot)_{m-\text { tors }}=\mathbb{H}_{\text {êt }}^{1}\left(\bar{X} \cdot, \mu_{m}\right)$.

Remark 4.4.4. - From Theorem 4.4.3 and Proposition 1.3.1, we can see that the definition of $\mathrm{Pic}^{+}(X)$ is independent of choices of hypercoverings and compactifications. In fact, given two such smooth proper hypercoverings of $X$ which admit smooth compactifications with normal crossing boundaries, we can always find a third one mapping to both, see [25, Exposé V bis, 5.1.7 and 5.2.4]. Now let $X$. be a smooth proper hypercovering of $X$ with smooth compactification $\bar{X}^{\prime}$., and let $X^{\prime \prime}$ be another one, with smooth compactification $\bar{X}^{\prime \prime}$. mapping to $\bar{X}^{\prime}$. compatibly with the normal crossing boundaries. Then, we get a map of 1-motives

$$
\left[\operatorname{Div}_{Y_{\bullet}^{\prime}}^{0}\left(\bar{X}^{\prime} \cdot\right) \rightarrow \operatorname{Pic}^{0}\left(\bar{X}^{\prime} \cdot\right)\right] \rightarrow\left[\operatorname{Div}_{Y^{\prime \prime}}^{0}\left(\bar{X}^{\prime \prime}\right) \rightarrow \operatorname{Pic}^{0}\left(\bar{X}^{\prime \prime} \cdot\right)\right]
$$

by pulling back cycles and simplicial line bundles. By the Theorem 4.4.3

$$
\widehat{T}\left(\left[\operatorname{Div}_{Y_{\bullet}^{\prime}}^{0}\left(\bar{X}^{\prime} \cdot\right) \rightarrow \operatorname{Pic}^{0}\left(\bar{X}^{\prime} \cdot\right)\right]\right) \cong \widehat{T}\left(\left[\operatorname{Div}_{Y^{\prime \prime}}^{0}\left(\bar{X}_{\bullet}^{\prime \prime}\right) \rightarrow \operatorname{Pic}^{0}\left(\bar{X}_{\bullet}^{\prime \prime}\right)\right]\right) .
$$

By Proposition 1.3.1 this isomorphism lifts to an isomorphism of 1-motives.

However, as for the case of $\mathrm{Pic}^{-}$, one would like to see directly, by a geometric argument, that the above map of 1-motives is an isomorphism.

\subsection{De Rham realization of $\mathrm{Pic}^{+}$}

Let $k$ be a field of characteristic 0 . For any simplicial $k$-scheme $X$. we will denote by $\operatorname{Pic}^{\natural}(X$.$) the group of isomorphism classes of pairs (\mathcal{L} ., \nabla \cdot)$, where $\mathcal{L}$. is a simplicial line bundle and $\nabla$. is a simplicial integrable connection

$$
\nabla .: \mathcal{L} . \rightarrow \mathcal{L} \cdot \otimes_{\mathcal{O}_{X}} \Omega_{X}^{1} \cdot
$$

We can consider the simplicial -Picard functor on the category of $k$-schemes, which we denote by $\mathbf{P i c}_{X}^{\natural} / k$, obtained by sheafifying the functor

$$
T \longmapsto \operatorname{Pic}^{\natural}\left(X \cdot \times_{k} T\right)
$$

with respect to the fpqc-topology.

For a given pair $(\mathcal{L} ., \nabla$.$) we clearly get a pair (\mathcal{L}, \nabla)$ on $X_{0}$ and an isomorphism $\alpha: d_{0}^{*}(\mathcal{L}, \nabla) \stackrel{\cong}{\longrightarrow} d_{1}^{*}(\mathcal{L}, \nabla)$, i.e., $\alpha$ is an isomorphism $\left(d_{0}\right)^{*}(\mathcal{L}) \stackrel{\cong}{\longrightarrow}\left(d_{1}\right)^{*}(\mathcal{L})$ which is compatible with the connections, and, moreover, $\alpha$ satisfies the cocycle condition (cf. Proposition 4.1.1). In fact, we have the following description.

Proposition 4.5.1. - Let X. be any smooth proper simplicial $k$-scheme. Elements of $\mathbf{P i c}{ }^{\natural}(X$.$) are in natural bijection with isomorphism classes of triples (\mathcal{L}, \nabla, \alpha)$ consisting of an invertible sheaf $\mathcal{L}$ on $X_{0}$, with an integrable connection $\nabla$, and an 
isomorphism $\alpha: d_{0}^{*}(\mathcal{L}, \nabla) \stackrel{\cong}{\longrightarrow} d_{1}^{*}(\mathcal{L}, \nabla)$ satisfying the cocycle condition. There is a functorial isomorphism

$$
\mathbf{P i c}^{\natural}(X .) \cong \mathbb{H}^{1}\left(X ., \mathcal{O}_{X}^{*} \stackrel{\text { dlog }}{\longrightarrow} \Omega_{X}^{1} .\right)
$$

Proof. - It follows from the Proposition 4.1 .1 and a simplicial version of $[\mathbf{3 2}$, Sections 3-4] according to the general hint given by Deligne in $[\mathbf{1 5}, 10.3 .10]$.

We have the following exact sequence

$$
0 \rightarrow \mathbb{H}^{0}\left(X ., \Omega_{X .}^{1}\right) \rightarrow \mathbf{P i c}^{\natural}(X .) \rightarrow \operatorname{Pic}(X .) \rightarrow \mathbb{H}^{1}\left(X ., \Omega_{X .}^{1}\right)
$$

which is obtained from the exact sequence of complexes of simplicial sheaves

$$
0 \rightarrow \Omega_{X}^{1}[-1] \rightarrow\left[\mathcal{O}_{X}^{*} \stackrel{\text { dlog }}{\longrightarrow} \Omega_{X .}^{1}\right] \rightarrow \mathcal{O}_{X}^{*} \rightarrow 0
$$

using the Proposition 4.5.1.

Since $X$. is smooth and proper over $k$, the semi-abelian variety $\mathbf{P i c}^{0}(X$.$) is mapped$ to zero in $\mathbb{H}^{1}\left(X ., \Omega_{X}^{1}\right.$. ; we thus get an extension

$$
0 \rightarrow \mathbb{H}^{0}\left(X ., \Omega_{X .}^{1}\right) \rightarrow \mathbf{P i c}^{\natural}(X .)^{0} \rightarrow \mathbf{P i c}^{0}(X .) \rightarrow 0
$$

by pulling back along the inclusion $\mathbf{P i c}^{0} \hookrightarrow$ Pic. The group extension (35) is the group of $k$-points of the universal $\mathbb{G}_{a}$-extension of the semi-abelian scheme $\mathbf{P i c} \mathbf{C}_{X / k}^{0}$; in fact we have the following.

Lemma 4.5.2. - Let $X$. be a smooth proper simplicial $k$-scheme, where $k$ is algebraically closed of characteristic 0 . We have that

$$
\left(\mathbf{P i c}_{X . / k}^{0}\right)^{\natural} \cong\left(\mathbf{P i c}_{X . / k}^{\natural}\right)^{0},
$$

and we have a canonical isomorphism

$$
\operatorname{Lie}\left(\mathbf{P i c}_{X . / k}^{\natural}\right)^{0} \cong \mathbb{H}^{1}\left(X ., \mathcal{O}_{X} . \rightarrow \Omega_{X}^{1}\right) .
$$

Proof. - The universal $\mathbb{G}_{a}$-extension of any semi-abelian scheme is obtained as a pull-back from the universal extension of its abelian quotient. The abelian quotient of $\mathbf{P i c}_{X . / k}^{0}$ is

$$
A_{X} . \stackrel{\text { def }}{=} \operatorname{ker}^{0}\left(\operatorname{Pic}_{X_{0} / k}^{0} \rightarrow \operatorname{Pic}_{X_{1} / k}^{0}\right) .
$$

By [32, Sections 3-4] it is easy to see that the universal $\mathbb{G}_{a}$-extension of $A_{X}$. is given by the group scheme ( $c f$. Chapter 1$)$

$$
\operatorname{ker}^{0}\left(\left(\operatorname{Pic}_{X_{0} / k}^{\natural}\right)^{0} \rightarrow\left(\operatorname{Pic}_{X_{1} / k}^{\natural}\right)^{0}\right)
$$

and we then have that

$$
\operatorname{Ext}\left(A_{X}, \mathbb{G}_{a}\right)^{\vee} \cong \operatorname{ker}\left(H^{0}\left(X_{0}, \Omega_{X_{0}}^{1}\right) \rightarrow H^{0}\left(X_{1}, \Omega_{X_{1}}^{1}\right)\right) .
$$


Everything then follows from the following diagram with exact rows and columns,

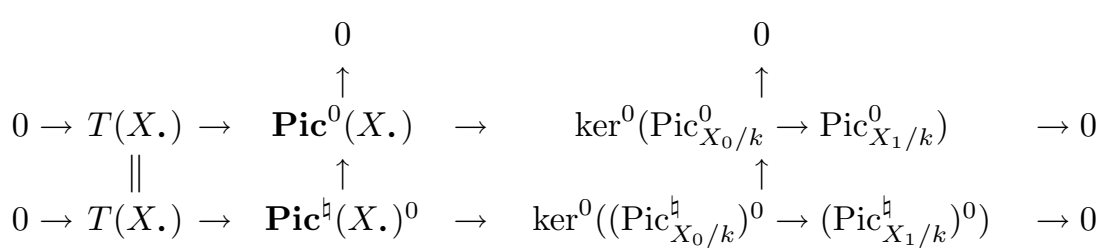

$$
\begin{aligned}
& \mathbb{H}^{0}\left(X_{\bullet}, \Omega_{X .}^{1}\right)=\operatorname{ker}\left(H^{0}\left(X_{0}, \Omega_{X_{0}}^{1}\right) \rightarrow H^{0}\left(X_{1}, \Omega_{X_{1}}^{1}\right)\right) \\
& \uparrow \quad \uparrow
\end{aligned}
$$

where $T(X$.$) is the toric part of \mathbf{P i c}_{X, / k}^{0}$ and the middle row and column are exact by Proposition 4.5.1 and (35). Therefore, by taking associated sheaves, we see that $\left(\mathbf{P i c}_{X . / k}^{\natural}\right)^{0}$ is representable by the pull-back of the universal extension of $A_{X}$. Finally, since the Lie algebra of $\mathbf{P i c}_{X . / k}^{0}$ is $H^{1}\left(X ., \mathcal{O}_{X}\right.$.), from (35), we get the last claim by taking Lie algebras (cf. [32]).

More generally, let $X$. be a smooth simplicial $k$-variety, where $k$ is a field of characteristic 0 . Let $\bar{X}$. be a smooth compactification with normal crossing boundary $Y$.

We then define $\mathbf{P} \mathbf{i c}^{\natural-\log }(X$.$) to be the group of isomorphism classes of pairs$ $\left(\mathcal{L} ., \nabla^{\log }\right)$, where $\mathcal{L}$. is a simplicial line bundle on $\bar{X}$ and $\nabla^{\log }$ is a simplicial integrable connection with $\log$ poles along $Y$. , i.e., $\nabla^{\log }$ is a $k$-linear simplicial sheaf homomorphism

$$
\nabla_{\cdot}^{\log }: \mathcal{L} \cdot \rightarrow \mathcal{L} \cdot \otimes_{\mathcal{O}_{X}}, \Omega_{\bar{X}}(\log Y \cdot)
$$

satisfying the Leibniz product rule (cf. [20, Section 2], [32, Section 3], [14]). We clearly have a natural injective homomorphism

$$
\operatorname{Pic}^{\natural}(\bar{X} .) \longrightarrow \mathbf{P i c}^{\natural-\log }(X .)
$$

and we have the following cohomological description.

Proposition 4.5.3. - Let X. be any smooth simplicial k-variety. Elements of the group $\mathbf{P i c} \mathbf{c}^{\natural-\log }(X$.$) are in bijection with isomorphism classes of triples \left(\mathcal{L}, \nabla^{\log }, \alpha\right)$ consisting of an invertible sheaf $\mathcal{L}$ on $\bar{X}_{0}$, with an integrable connection with log poles

$$
\nabla^{\log }: \mathcal{L} \rightarrow \mathcal{L} \otimes_{\mathcal{O}_{X_{0}}} \Omega \bar{X}_{\bar{X}_{0}}\left(\log Y_{0}\right)
$$

and an isomorphism $\alpha: d_{0}^{*}\left(\mathcal{L}, \nabla^{\log }\right) \stackrel{\cong}{\rightrightarrows} d_{1}^{*}\left(\mathcal{L}, \nabla^{\log }\right)$, satisfying the cocycle condition. There is a functorial isomorphism

$$
\mathbf{P i c}^{\natural-\log }\left(X_{\bullet}\right) \cong \mathbb{H}^{1}\left(\bar{X} ., \mathcal{O}_{\bar{X}}^{*} . \stackrel{\operatorname{dlog}}{\longrightarrow} \Omega_{\bar{X}}^{1}(\log Y \cdot)\right)
$$

Proof. - A variant of the proof of Proposition 4.5.1 (cf. Lemma 2.6.2). 
Now let $X$ be a $k$-variety, where $k$ is a field of characteristic 0 . Let $X . \rightarrow X$ be a smooth proper hypercovering, and $\bar{X}$. a smooth proper compactification with normal crossing boundary $Y$.. We recall (2.6.3) that by the De Rham cohomology of $X$ we mean the graded, filtered $k$-vector space

$$
H_{\mathrm{DR}}^{*}(X) \stackrel{\text { def }}{=} \mathbb{H}^{*}\left(\bar{X} \cdot, \Omega_{\bar{X}}^{\cdot}(\log Y \cdot)\right)
$$

THEOREM 4.5.4. - Let $X$ be a k-variety, where $k$ is algebraically closed of characteristic 0 . Then

$$
T_{\mathrm{DR}}\left(\operatorname{Pic}^{+}(X)\right) \cong H_{\mathrm{DR}}^{1}(X)(1) .
$$

Proof. - Let $\operatorname{Pic}^{+}(X)$ be given by the 1-motive $\left[\operatorname{Div}_{Y}^{0}(\bar{X} \cdot) \rightarrow \mathbf{P i c}^{0}(\bar{X} \cdot)\right]$ for a chosen hypercovering and compactification with normal crossing boundary $Y$.. We have the following exact sequence of complexes

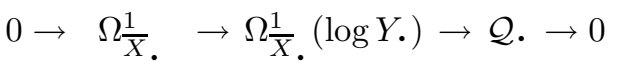

$$
\begin{aligned}
& \uparrow \operatorname{dlog} \quad \uparrow \operatorname{dlog} \uparrow \\
& 0 \rightarrow \mathcal{O}_{\bar{X}}^{*}=\mathcal{O}_{\bar{X}}^{*} . \quad \rightarrow 0
\end{aligned}
$$

where $\mathcal{Q}$. is just the quotient $\Omega_{\bar{X}}^{1}{ }_{. / k}\left(\log Y_{\bullet}\right) / \Omega_{\bar{X}}^{1}{ }_{. / k}$. We therefore have the following push-out diagram

$$
\begin{aligned}
& 0 \rightarrow \quad \mathbb{H}^{0}\left(\bar{X} \cdot, \Omega_{\bar{X}}{ }^{\prime}\right) \quad \rightarrow \quad \mathbf{P i c}^{\natural}(\bar{X} \cdot)^{0} \quad \rightarrow \mathbf{P i c}^{0}(\bar{X} \cdot) \rightarrow 0 \\
& 0 \rightarrow \mathbb{H}^{0}\left(\bar{X} \cdot, \stackrel{\downarrow}{\downarrow} \frac{1}{X}_{.}(\log Y .)\right) \rightarrow \mathbf{P i c}^{\natural-\log }(X .)^{0} \rightarrow \mathbf{P i c}^{0}(\bar{X} \cdot) \rightarrow 0
\end{aligned}
$$

where the top row is (35) and the bottom row is obtained from the dlog map as well. From the latter we are then left to show that

$$
\operatorname{Ext}\left(\operatorname{Pic}^{+}, \mathbb{G}_{a}\right)^{\vee}=\mathbb{H}^{0}\left(\bar{X} \cdot, \Omega_{\bar{X}}^{1}(\log Y \cdot)\right) .
$$

In fact, granting (36), we have that, by the push-out diagram and the Lemma 4.5.2, the universal $\mathbb{G}_{a}$-extension of $\operatorname{Pic}^{+}(X)$ is given by

$$
\operatorname{Pic}^{+}(X)^{\natural} \cong\left[\operatorname{Div}_{Y \cdot}^{0}(\bar{X} \cdot) \stackrel{u^{\natural}}{\longrightarrow} \operatorname{Pic}^{\natural-\log }(X \cdot)^{0}\right]
$$


where the lifting $u^{\natural}$ above of $u: \operatorname{Div}_{Y_{\bullet}}^{0}(\bar{X} \cdot) \rightarrow \mathbf{P i c}^{0}(\bar{X} \cdot)$ can be described as in Lemma 2.6.2 via the Proposition 4.5.3. Therefore

$$
\begin{aligned}
T_{\mathrm{DR}}\left(\operatorname{Pic}^{+}(X)\right) & \stackrel{\text { def }}{=} \operatorname{Lie} \mathbf{P i c}^{\natural-\log }(X \cdot)^{0} \\
& \cong \operatorname{Lie}\left(\mathbb{H}^{1}\left(\bar{X} \cdot, \mathcal{O}_{\bar{X}}^{*} \cdot \stackrel{\operatorname{dlog}}{\longrightarrow} \Omega \frac{1}{\bar{X}} \cdot(\log Y \cdot)\right)\right. \\
& \cong \mathbb{H}^{1}\left(\bar{X} \cdot, \mathcal{O}_{\bar{X}} \rightarrow \Omega_{\bar{X}}(\log Y \cdot)\right) \\
& \cong \mathbb{H}^{1}\left(\bar{X} \cdot, \Omega_{\bar{X}}^{\cdot}(\log Y \cdot)\right) \\
& \stackrel{\text { def }}{=} H_{\mathrm{DR}}^{1}(X)
\end{aligned}
$$

Moreover, this isomorphism is clearly compatible with the Hodge filtrations, provided we shift the index of the filtration on the right by 1 .

In order to show (36) we consider the following commutative diagram with exact columns

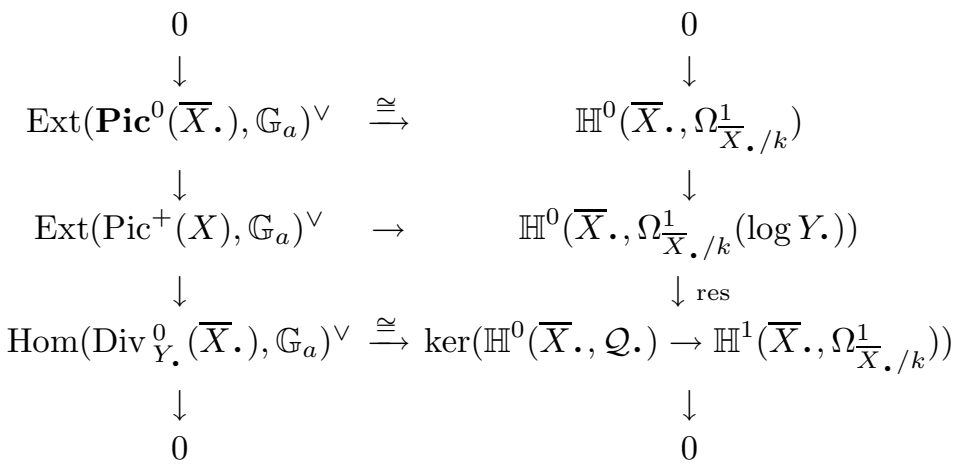

where the horizontal maps are the canonical maps induced by universality; from the previous Lemma 4.5.2 we know that the horizontal map on top is an isomorphism, so that we are left to show that the horizontal map at the bottom is an isomorphism.

If $Y$. is smooth this last claim is clear since we have a simplicial surjective Poincaré residue map

$$
\text { res. : } \Omega \frac{1}{X} \cdot\left(\log Y_{\bullet}\right) \rightarrow \mathcal{O}_{Y_{\bullet}},
$$

and therefore $\mathbb{H}^{0}(\bar{X} ., \mathcal{Q}.) \cong \mathbb{H}^{0}\left(Y_{.}, \mathcal{O}_{Y_{\text {. }}}\right)$. In general, since the subschemes $Y_{i} \subset X_{i}$ are normal crossing divisors, for each $i \geqslant 0$, we have exact sequences $(c f$. [20, 2.3])

$$
0 \rightarrow \Omega \frac{1}{\bar{X}_{i}} \rightarrow \Omega \frac{1}{\bar{X}_{i}}\left(\log Y_{i}\right) \rightarrow \oplus_{j i} \mathcal{O}_{Y_{j i}} \rightarrow 0
$$

where the index $j i$ ( $i$ fixed) runs over the smooth components of $Y_{i}$, i.e., $Y_{i}=\cup_{j i} Y_{j i}$. These sequences are compatible via the face and degeneracy maps of the simplicial scheme $\bar{X}$. B Because of this construction, and the definition of global sections of a 
simplicial sheaf, we clearly get a canonical identification

$$
\mathbb{H}^{0}(\bar{X} ., \mathcal{Q} .)=\operatorname{ker}\left(\oplus_{j 0} H^{0}\left(Y_{j 0}, \mathcal{O}_{Y_{j 0}}\right) \rightarrow \oplus_{j 1} H^{0}\left(Y_{j 1}, \mathcal{O}_{Y_{j 1}}\right)\right) \cong \operatorname{Div}_{Y}(\bar{X} .) \otimes k .
$$

We finally then get $\operatorname{Div}_{Y_{\bullet}}^{0}(\bar{X} \cdot) \otimes k \cong \operatorname{ker}\left(\operatorname{Div}_{Y_{\bullet}}(\bar{X} \cdot) \otimes k \rightarrow \mathbb{H}^{1}\left(\bar{X} ., \Omega \frac{1}{X_{\bullet}}\right)\right)$ as claimed.

REMARK 4.5.5. - From Theorem 4.5.4, we obtain an "algebraic proof" (i.e., independent of base change to $\mathbb{C}$ and comparison with the analytic topology) that the filtered vector space $\left(H_{\mathrm{DR}}^{1}(X), F\right)$ is independent of the choices of $X$. and its compactification $\bar{X}$., since the 1-motive $\mathrm{Pic}^{+}$is independent of these choices, as we saw earlier using étale realizations, as a consequence of Theorem 4.4.3.

REMARK 4.5.6. - For a given singular variety $X$ we can consider a singular compactification $\bar{X}$ in such a way that $\bar{X}$., in our above considerations, is a hypercovering of $\bar{X}$. By the previous argument, in the proof of the Theorem 4.5.4, we constructed the following extension

$$
\begin{aligned}
& 0 \rightarrow T_{\mathrm{DR}}\left(\operatorname{Pic}^{0}(\bar{X} .)\right) \rightarrow T_{\mathrm{DR}}\left(\operatorname{Pic}^{+}(X)\right) \rightarrow T_{\mathrm{DR}}\left(\operatorname{Div}_{Y_{\bullet}}^{0}(\bar{X} \cdot)[1]\right) \rightarrow 0 \\
& \cong \uparrow \quad \cong \uparrow \quad \cong \uparrow \\
& 0 \rightarrow \quad H_{\mathrm{DR}}^{1}(\bar{X})(1) \quad \rightarrow \quad H_{\mathrm{DR}}^{1}(X)(1) \stackrel{\text { res }}{\longrightarrow} \operatorname{Div}_{Y \cdot}^{0}(\bar{X} \cdot) \otimes k \quad \rightarrow 0
\end{aligned}
$$

The resulting bottom row can be regarded as obtained from an exact sequence of cohomology with supports as well as a Poincaré "residue" map compatible with the Hodge filtration. 


\section{CHAPTER 5}

\section{HOMOLOGICAL ALBANESE 1-MOTIVE: Alb}

We keep the notations and hypotheses from the previous chapter.

\subsection{Definition of $\mathrm{Alb}^{-}$}

In order to define our homological Albanese $\mathrm{Alb}^{-}(X)$ we just take the Cartier dual of $\operatorname{Pic}^{+}(X)$.

Definition 5.1.1. - If $X$ is a variety over an algebraically closed field $k$ of characteristic zero, define the following 1-motive

$$
\operatorname{Alb}^{-}(X) \stackrel{\text { def }}{=} \operatorname{Pic}^{+}(X)^{\vee}=\left[\operatorname{Div}_{Y}^{0}(\bar{X} \cdot) \rightarrow \operatorname{Pic}^{0}(\bar{X} \cdot)\right]^{\vee},
$$

where $X . \rightarrow X$ is a smooth proper hypercovering, and $\bar{X}$. a smooth compactification of $X$. with normal crossing boundary $Y$.. We call $\mathrm{Alb}^{-}(X)$ the homological Albanese 1-motive of $X$.

Lemma 5.1.2. - If $X$ is proper over $k=\bar{k}$ of characteristic 0 , and $\pi: X . \rightarrow X$ is any proper hypercovering, then the natural homomorphism between connected algebraic $k$-groups

$$
\pi^{*}: \operatorname{Pic}^{0}(X) \rightarrow \operatorname{Pic}^{0}(X .)
$$

is a surjection with torsion free kernel.

Proof. - In fact, by Kummer theory and cohomological descent we get the following commutative square of isomorphisms

$$
\begin{gathered}
\mathbb{H}_{\text {ét }}^{1}\left(X ., \mu_{m}\right) \stackrel{\cong}{\cong} \operatorname{Pic}(X \cdot)_{m-\text { tors }} \\
\quad \cong \uparrow \\
H_{\text {ét }}^{1}\left(X, \mu_{m}\right) \stackrel{\cong}{\cong} \operatorname{Pic}(X)_{m-\text { tors }}
\end{gathered}
$$

Therefore, since the Néron-Severi groups are finitely generated, the Tate module of $\operatorname{Pic}^{0}(X)$ is isomorphic to $\widehat{T}\left(\mathbf{P} \mathbf{i c}^{0}(X\right.$. $\left.)\right)$. To conclude we remark that $\mathbf{P i c}{ }^{0}(X$. $)$ is the 
group of $k$-points of a semi-abelian variety, in which torsion points are Zariski dense (cf. Proposition 1.3.1).

REMARK 5.1.3. - As a consequence, we see that for any smooth proper hypercovering $X . \rightarrow X$ of a proper $k$-variety $X$, the simplicial Picard variety $\mathbf{P i c}^{0}(X$. $)$ is the semi-abelian quotient of the connected commutative algebraic group $\operatorname{Pic}^{0}(X)$.

For a proper smooth hypercovering $\pi: X$. $\rightarrow X$ of a proper $k$-scheme $X$, where $k=\bar{k}$, we let $\mathbb{Z}^{X_{i}}$ denote the free abelian group on connected components of $X_{i}$. Let

$$
L_{X} . \stackrel{\text { def }}{=} \frac{\operatorname{ker}\left(\mathbb{Z}^{X_{1}} \rightarrow \mathbb{Z}^{X_{0}}\right)}{\operatorname{im}\left(\mathbb{Z}^{X_{2}} \rightarrow \mathbb{Z}^{X_{1}}\right)} \quad(\bmod \text { torsion })
$$

and consider the following abelian variety

$$
\left(\operatorname{ker}^{0}\left(\operatorname{Pic}^{0}\left(X_{0}\right) \stackrel{d_{0}^{*}-d_{1}^{*}}{\longrightarrow} \operatorname{Pic}^{0}\left(X_{1}\right)\right)\right)^{\vee}=\frac{\operatorname{Alb}\left(X_{0}\right)}{\left(d_{0}-d_{1}\right)_{*} \operatorname{Alb}\left(X_{1}\right)} .
$$

Here $\left(d_{0}-d_{1}\right)_{*} \stackrel{\text { def }}{=}\left(d_{0}\right)_{*}-\left(d_{1}\right)_{*}: \operatorname{Alb}\left(X_{1}\right) \rightarrow \operatorname{Alb}\left(X_{0}\right)$.

Let $\left(d_{0}\right)_{*}-\left(d_{1}\right)_{*}: \mathcal{Z}_{0}\left(X_{1}\right) \rightarrow \mathcal{Z}_{0}\left(X_{0}\right)$ denote as well the induced map between zero-cycles, and let $a_{X_{0}}: \mathcal{Z}_{0}\left(X_{0}\right)_{0} \rightarrow \operatorname{Alb}\left(X_{0}\right)$ be the Albanese map, defined on the subgroup $\mathcal{Z}_{0}\left(X_{0}\right)_{0} \subset \mathcal{Z}_{0}\left(X_{0}\right)$ of zero-cycles on $X_{0}$ which have degree zero on each component of $X_{0}$.

The Albanese variety of the smooth, proper $k$-variety $X_{0}$ is here defined to be the product of the Albanese varieties of its connected components (note that these connected components are irreducible, smooth, proper $k$-varieties, which need not have the same dimension).

Proposition 5.1.4. - Let $X$ be proper over $k=\bar{k}$. Then $\mathrm{Alb}^{-}(X)$ coincides with the 1-motive

$$
u_{X}: L_{X} \rightarrow \frac{\operatorname{Alb}\left(X_{0}\right)}{\left(d_{0}-d_{1}\right)_{*} \operatorname{Alb}\left(X_{1}\right)}
$$

where the map $u_{X}$. is defined as follows. For each connected component $X^{c}$ of $X_{1}$ choose a closed point $x_{c} \in X^{c}$. Then, for $\sum n_{c} X_{c} \in \operatorname{ker}\left(\mathbb{Z}^{X_{1}} \rightarrow \mathbb{Z}^{X_{0}}\right)$, we have

$$
\sum n_{c}\left(\left(d_{0}\right)_{*}\left(x_{c}\right)-\left(d_{1}\right)_{*}\left(x_{c}\right)\right) \in \mathcal{Z}_{0}\left(X_{0}\right)_{0},
$$

and we define

$$
\begin{aligned}
u_{X} .\left(\sum n_{c} X_{c}\right. & \left.\left(\bmod \operatorname{im} \mathbb{Z}^{X_{2}}\right)\right) \\
& \stackrel{\text { def }}{=} a_{X_{0}}\left(\sum n_{c}\left(d_{0}\right)_{*}\left(x_{c}\right)-\left(d_{1}\right)_{*}\left(x_{c}\right)\right) \quad\left(\bmod \left(d_{0}-d_{1}\right)_{*} \operatorname{Alb}\left(X_{1}\right)\right) .
\end{aligned}
$$

If $X$ is also normal then $L_{X}=0$ and

$$
\operatorname{Alb}^{-}(X) \cong \operatorname{Pic}^{0}(X)^{\vee}
$$

is an abelian variety. 
Proof. - To check that $u_{X}$. is well defined is left as an exercise. We recall that $\mathbf{P i c}^{0}(X$.$) is an extension of the abelian variety \operatorname{ker}^{0}\left(\operatorname{Pic}^{0}\left(X_{0}\right) \rightarrow \operatorname{Pic}^{0}\left(X_{1}\right)\right)$ by the torus $T(X$.$) (see (31) in Chapter 4$ for a description of the torus). Now, $L_{X}$. is the character group of the torus $T(X),. \operatorname{Alb}\left(X_{0}\right) / \mathrm{im} \operatorname{Alb}\left(X_{1}\right)$ is the dual abelian variety; the claimed map between them is obtained from Cartier duality - as in the proof of the corresponding assertion of Proposition 3.1.4, using standard functoriality properties of Albanese and Picard varieties, one can reduce to the case of the standard smooth proper hypercovering of an irreducible projective curve with 1 node; now we further reduce to determining the Cartier dual of $\mathrm{Pic}^{0}$ of this singular curve, which is treated in $[\mathbf{5 3}]$.

If $X$ is normal, then $\pi_{*}\left(\mathcal{O}_{X}^{*}\right)=\mathcal{O}_{X}^{*}$, and so

$$
\pi^{*}: \operatorname{Pic}(X) \hookrightarrow \operatorname{Pic}(X .)
$$

is injective, by the Leray spectral sequence for the sheaf $\mathcal{O}_{X}^{*}$. along $\pi$; therefore, from Lemma 5.1.2 we get

$$
\pi^{*}: \operatorname{Pic}^{0}(X) \cong \operatorname{Pic}^{0}(X .) .
$$

Since $X$ is normal, $\operatorname{Pic}^{0}(X)$ is an abelian variety [13], therefore $T(X \cdot)=0$.

Remark 5.1.5. - If $X$ is smooth, possibly open, then $X ., \bar{X} ., Y$. can be taken to be the constant simplicial schemes associated to $X, \bar{X}$ and $Y$, respectively, where $\bar{X}$ is a smooth compactification of $X$ with normal crossing boundary $Y$. In this case $\mathrm{Alb}^{-}(X)$ is a semi-abelian variety, which can be represented by an extension

$$
1 \rightarrow T(Y) \rightarrow \operatorname{Alb}^{-}(X) \rightarrow \operatorname{Alb}(\bar{X}) \rightarrow 0
$$

where, by definition, $T(Y)$ is the $k$-torus with character $\operatorname{group}_{\operatorname{Div}}^{0}(\bar{X})$; see Proposition 5.2.1 below.

REMARK 5.1.6. - As a consequence of Lemma 5.1.2 we have that

$$
H^{1}\left(X, \mathcal{O}_{X}\right) \rightarrow H^{1}\left(X, \pi_{*}\left(\mathcal{O}_{X} .\right)\right)
$$

is always a surjection, and the following edge homomorphism

$$
\mathbb{H}^{1}\left(X ., \mathcal{O}_{X} .\right) \stackrel{\text { zero }}{\longrightarrow} H^{0}\left(X, R^{1} \pi_{*}\left(\mathcal{O}_{X} .\right)\right)
$$

is the zero map. In fact, since $H^{1}\left(X, \mathcal{O}_{X}\right)=\operatorname{LiePic}^{0}(X)$ and $\mathbb{H}^{1}\left(X ., \mathcal{O}_{X}\right)=$ Lie $\mathbf{P i c}^{0}\left(X\right.$. ), we see that $H^{1}\left(X, \mathcal{O}_{X}\right)$ always surjects onto $\mathbb{H}^{1}\left(X ., \mathcal{O}_{X}\right)$; moreover,

$$
H^{1}\left(X, \mathcal{O}_{X}\right) \cong \mathbb{H}^{1}\left(X ., \mathcal{O}_{X} .\right)
$$

if $X$ is normal, and we then have that

$$
\operatorname{Lie} \operatorname{Alb}^{-}(X)=H^{1}\left(X, \mathcal{O}_{X}\right)^{\vee} .
$$




\subsection{Albanese mappings to $\mathrm{Alb}^{-}$}

Let $X_{\text {reg }}$ be the smooth locus of an equidimensional $k$-variety $X$, where $k$ is algebraically closed of characteristic 0 . We then have that $X_{\mathrm{reg}}=\widetilde{X}-\widetilde{S}=\bar{X}-(Y \cup \bar{S})$ for a resolution of singularities $\widetilde{X}$ and a good normal crossing compactification $\bar{X}$, with boundary divisor $Y$; also $Y \cup \bar{S}$ is a normal crossing divisor in $\bar{X}$.

We then obtain a commutative square of 1-motives

$$
\begin{aligned}
& {\left[0 \rightarrow \operatorname{Pic}^{0}(\bar{X}, Y)\right] \quad \rightarrow \quad\left[\operatorname{Div}_{Y}^{0}(\bar{X}) \rightarrow \operatorname{Pic}^{0}(\bar{X})\right]} \\
& {\left[\operatorname{Div} \frac{0}{S / S}(\bar{X}, Y) \rightarrow \operatorname{Pic}^{0}(\bar{X}, Y)\right] \rightarrow\left[\operatorname{Div} \frac{0}{S} \cup Y(\bar{X}) \rightarrow \operatorname{Pic}^{0}(\bar{X})\right]}
\end{aligned}
$$

which we may rewrite as

$$
\begin{array}{cc}
\operatorname{Pic}^{-}(\widetilde{X}) & \longrightarrow \operatorname{Pic}^{+}(\widetilde{X}) \\
\downarrow & \downarrow \\
\operatorname{Pic}^{-}(X) & \longrightarrow \operatorname{Pic}^{+}\left(X_{\text {reg }}\right) .
\end{array}
$$

By taking Cartier duals, we obtain the following commutative square.

$$
\begin{array}{cc}
\operatorname{Alb}_{\uparrow}^{-}(\tilde{X}) & \longrightarrow \operatorname{Alb}^{+}(\tilde{X}) \\
\uparrow & \uparrow \\
\operatorname{Alb}^{-}\left(X_{\mathrm{reg}}\right) & \longrightarrow \operatorname{Alb}^{+}(X) .
\end{array}
$$

In particular we get a canonical mapping

$$
\tau_{+}^{-}: \mathrm{Alb}^{-}\left(X_{\mathrm{reg}}\right) \rightarrow \mathrm{Alb}^{+}(X) .
$$

Let $a_{\mathbf{x}}: \bar{X} \rightarrow \operatorname{Alb}(\bar{X})$ be the Albanese mapping, obtained by choosing a base point $x_{c}$ in each component $X^{c}$ of $X_{\text {reg }}$ (note that the components of $X_{\text {reg }}$ and $\bar{X}$ are in bijection). Since $\mathrm{Alb}^{-}\left(X_{\text {reg }}\right)$ is a torus bundle on $\operatorname{Alb}(\bar{X})$ we can consider the following pull-back ( $c f$. Chapter 3)

$$
\begin{array}{ccc}
\operatorname{Alb}^{-}\left(X_{\text {reg }}\right) & \rightarrow & \operatorname{Alb}(\bar{X}) \\
\bar{a} \uparrow & & \uparrow a \\
\operatorname{Alb}^{T}(X) & \rightarrow & \bar{X}
\end{array}
$$

One can see that the torus bundle $\operatorname{Alb}^{T}(X) \rightarrow \bar{X}$ is trivial when restricted to the open subset $X_{\text {reg }} \subset \bar{X}$ (in fact, the same argument in Chapter 3 applies here, cf. [48, $\S 1])$. Hence we get a section $\sigma^{\text {reg }}: X_{\text {reg }} \rightarrow \operatorname{Alb}^{T}(X)$. By composing $\sigma^{\text {reg }}$ and $\bar{a}$ we get the Albanese mapping

$$
a_{\mathbf{x}}^{-}: X_{\text {reg }} \rightarrow \operatorname{Alb}^{-}\left(X_{\text {reg }}\right) .
$$

Proposition 5.2.1. - For any equidimensional variety $X$ over $k=\bar{k}$, the morphism

$$
a_{\mathrm{x}}^{-}: X_{\mathrm{reg}} \rightarrow \mathrm{Alb}^{-}\left(X_{\mathrm{reg}}\right)
$$


is universal among (base point preserving) morphisms to semi-abelian varieties, in the sense of Serre [47]. If $X$ is a normal proper $k$-variety, then $\mathrm{Alb}^{-}\left(X_{\mathrm{reg}}\right)$ is the Albanese variety of any resolution of singularities of $X$.

Proof. - It follows from the explicit construction by Serre in [48] that $\mathrm{Alb}^{-}\left(X_{\mathrm{reg}}\right)$ is equal to its "Albanese variety" in the sense of $[\mathbf{4 7}]$, and the morphism $a_{\mathbf{x}}^{-}$is then universal by [48, Théorème 1], i.e., any torus bundle on $\operatorname{Alb}(\bar{X})$ which is trivial on $X_{\text {reg }}$ is a push-out of $\mathrm{Alb}^{-}\left(X_{\text {reg }}\right)$. If moreover $X$ is normal and proper, and $\widetilde{X} \rightarrow X$ is a projective resolution of singularities, then $\operatorname{Div} \underset{S}{0}(\widetilde{X})=0$, as seen in the proof of Proposition 3.1.5; therefore the character group of the torus vanishes.

If $X$ is proper then $\mathrm{Alb}^{+}(X)$ is semi-abelian and the Albanese map

$$
a_{\mathbf{x}}^{+}: X_{\text {reg }} \rightarrow \operatorname{Alb}^{+}(X)
$$

defined in (29) can be obtained by composing $a_{\mathbf{x}}^{-}$and $\tau_{+}^{-}$defined in (37). Since $\mathrm{Alb}^{-}\left(X_{\text {reg }}\right)$ is universal, $\tau_{+}^{-}$can be also be regarded as being induced by the universal property (note that $\tau_{+}^{-}$is affine and surjective).

Proposition 5.2.2. - Let $X$ be proper over $k$. Then there is an extension

$$
0 \rightarrow T(S) \rightarrow \mathrm{Alb}^{-}\left(X_{\mathrm{reg}}\right) \rightarrow \mathrm{Alb}^{+}(X) \rightarrow 0
$$

with kernel the torus $T(S)$ whose character group is the quotient lattice

$$
\frac{\operatorname{Div} \underset{\widetilde{S}}{\widetilde{S}}(\tilde{X})}{\operatorname{Div} \underset{\widetilde{S} / S}{0}(\widetilde{X})} .
$$

This is a sublattice of the lattice of divisors on $X$ which are supported on the singular locus $S$ of $X$ : in particular, $T(S)=0$ if $X$ is non-singular in codimension one.

Proof. - Since $\bar{X}=\widetilde{X}$, the claimed torus bundle is obtained as the Cartier dual of the following injective map of 1-motives

$$
\left[\operatorname{Div} \underset{\widetilde{S} / S}{0}(\widetilde{X}) \rightarrow \operatorname{Pic}^{0}(\widetilde{X})\right] \rightarrow\left[\operatorname{Div} \underset{\widetilde{S}}{0}(\bar{X}) \rightarrow \operatorname{Pic}^{0}(\widetilde{X})\right]
$$

Since $\operatorname{Div}_{\widetilde{S} / S}(\widetilde{X}) \stackrel{\text { def }}{=} \operatorname{ker}\left(\operatorname{Div}_{\widetilde{S}}(\widetilde{X}) \rightarrow \operatorname{Div}_{S}(X)\right)$ the description of $T(S)$ is clear.

\subsection{Hodge, étale and De Rham realizations of $\mathrm{Alb}^{-}$}

An immediate consequence of the Theorem 4.3.2 is the following.

Corollary 5.3.1. — Let $X$ be defined over $\mathbb{C}$. Then

$$
T_{\text {Hodge }}\left(\operatorname{Alb}^{-}(X)\right) \cong H_{1}(X, \mathbb{Z}) /(\text { torsion })
$$


Proof. - It follows from Cartier duality and the isomorphism of mixed Hodge structures

$$
\operatorname{Hom}\left(H^{1}(X, \mathbb{Z}(1)), \mathbb{Z}(1)\right) \cong H_{1}(X, \mathbb{Z}) /(\text { torsion })
$$

because of Theorem 4.3.2.

As a consequence of Theorems 4.4.3 and 4.5.4 we have:

Corollary 5.3.2. - Let $X$ be defined over an algebraically closed field of characteristic zero. Then

$$
\widehat{T}\left(\operatorname{Alb}^{-}(X)\right) \cong H_{1}^{e ́ t}(X, \widehat{\mathbb{Z}}) /(\text { torsion })
$$

and

$$
T_{\mathrm{DR}}\left(\operatorname{Alb}^{-}(X)\right) \cong H_{1}^{D R}(X) .
$$

We then have the following corollary deduced from the properties of $\mathrm{Alb}^{-}$and $\mathrm{Alb}^{+}$ obtained so far (see Proposition 5.2.2 and Lemma 5.1.2). Of course this may also be proved directly by topological arguments (and is in fact well known to experts).

Corollary 5.3.3. - Let $X$ be a normal proper $k$-variety. Then

$$
H_{1}^{e ́ t}\left(X_{\text {reg }}, \widehat{\mathbb{Z}}\right) /(\text { torsion }) \cong H_{1}^{e ́ t}(\widetilde{X}, \widehat{\mathbb{Z}}) /(\text { torsion })
$$

and $H_{1}^{e t}(X, \widehat{\mathbb{Z}}) /($ torsion $)$ is a quotient of $H_{1}^{e t}(\widetilde{X}, \widehat{\mathbb{Z}})$.

If $k=\mathbb{C}$ then

$$
H_{1}\left(X_{\mathrm{reg}}, \mathbb{Z}\right) /(\text { torsion }) \cong H_{1}(\widetilde{X}, \mathbb{Z}) /(\text { torsion })
$$

are isomorphic Hodge structures, pure of weight -1 , and $H_{1}(X, \mathbb{Z}) /($ torsion $)$ is a quotient Hodge structure of $H_{1}(\widetilde{X}, \mathbb{Z})$. 


\section{CHAPTER 6}

\section{MOTIVIC ABEL-JACOBI AND GYSIN MAPS}

We now obtain some further properties of our Albanese and Picard 1-motives. We will give algebro-geometric (= "motivic") constructions of some cohomological operations. We will work throughout over an algebraically closed base field $k$ of characteristic 0 .

\subsection{Semi-normalization}

Deligne's original construction of the motivic cohomology of a curve ( $c f$. Proposition 3.1.2) shows that it depends only on the semi-normalization of the curve, and not on the curve itself. For example, the motivic cohomology of the cuspidal projective plane cubic is zero. This is due to the fact that, e.g., over $\mathbb{C}$, the singular cohomology groups, compatibily with their mixed Hodge structures, are invariant under homeomorphisms.

In general, we easily get the following result in arbitrary dimension.

Proposition 6.1.1. - Let $f: X \rightarrow X^{\prime}$ be a radicial $k$-morphism such that $f(X)=$ $X^{\prime}$, i.e., $f_{K}: X(K) \rightarrow X^{\prime}(K)$ is bijective on $K$-points for any field extension $k \subseteq$ $K$. Then there are canonical isomorphisms $\operatorname{Pic}^{-}(X) \cong \operatorname{Pic}^{-}\left(X^{\prime}\right)$ and $\operatorname{Pic}^{+}(X) \cong$ $\operatorname{Pic}^{+}\left(X^{\prime}\right)$, thus, dually, $\operatorname{Alb}^{+}(X) \cong \operatorname{Alb}^{+}\left(X^{\prime}\right)$ and $\operatorname{Alb}^{-}(X) \cong \operatorname{Alb}^{-}\left(X^{\prime}\right)$.

Proof. - In fact this is a direct consequence of our definitions. For example, we can take a resolution of singularities $\widetilde{X}$ of $X$ which is a resolution of $X^{\prime}$ as well. Thus the 1-motives $\mathrm{Pic}^{-}(X)$ and $\mathrm{Pic}^{-}\left(X^{\prime}\right)$ are clearly isomorphic by construction. Similarly, if $\pi: X . \rightarrow X$ is a smooth proper hypercovering of $X$, there is an induced one $\pi^{\prime}: X . \rightarrow X^{\prime}$, yielding the identification $\operatorname{Pic}^{+}(X) \cong \operatorname{Pic}^{*}\left(X^{\prime}\right)$.

In particular, all four of our 1-motives associated to a $k$-variety $X$, in fact depend only on the semi-normalization of $X$. 


\subsection{Functoriality}

Contravariant (resp. covariant) functoriality of $\mathrm{Pic}^{+}$(resp. Alb ${ }^{-}$) is true, essentially by construction, and is valid for every morphism. For Pic ${ }^{-}\left(\mathrm{resp} . \mathrm{Alb}^{+}\right)$we can expect covariance (resp. contravariance) only for morphisms between varieties of the same dimension, yielding the zero map if the morphism does not have dense image in some irreducible component.

Proposition 6.2.1. - Let $f: X \rightarrow X^{\prime}$ be any morphism between $k$-varieties such that $\operatorname{dim} X^{\prime}=\operatorname{dim} X$. We then have a push-forward $f_{*}: \mathrm{Pic}^{-}(X) \rightarrow \mathrm{Pic}^{-}\left(X^{\prime}\right)$ and, dually, a pull-back $f^{*}: \operatorname{Alb}^{+}\left(X^{\prime}\right) \rightarrow \operatorname{Alb}^{+}(X)$.

Proof. - We will assume, for simplicity of exposition, that $X$ and $X^{\prime}$ are irreducible; we leave the necessary modifications (mainly notational) for the general case to the reader.

If the morphism $f$ is not dominant we define $f_{*}$ to be the zero homomorphism. If $f$ is dominant, we choose resolutions $\widetilde{X} \rightarrow X, \widetilde{X}^{\prime} \rightarrow X^{\prime}$ and good compactifications $\widetilde{X} \hookrightarrow \bar{X}$ and $\widetilde{X}^{\prime} \hookrightarrow \bar{X}^{\prime}$ with normal crossing boundaries $Y \subset \bar{X}, Y^{\prime} \subset \bar{X}^{\prime}$, such that there is a morphism $\bar{f}: \bar{X} \rightarrow \bar{X}^{\prime}$ compatible with $f$, and hence satisfying $\bar{f}^{-1}\left(Y^{\prime}\right) \subset Y$.

Let $D \in \operatorname{Div}_{\bar{S} / S}(\bar{X}, Y)$. The push-forward $f_{*}(D)$, as a Weil divisor, clearly belongs to $\operatorname{Div}_{S^{\prime} / S^{\prime}}\left(\bar{X}^{\prime}, Y^{\prime}\right)$. We therefore just need to show that there is an induced pushforward of relative line bundles which is compatible with the push-forward of Weil divisors. This is the content of the following lemma.

Lemma 6.2.2. - Let $f: \bar{X} \rightarrow \overline{X^{\prime}}$ be a proper surjective morphism between $n$ dimensional integral smooth proper varieties over an algebraically closed field $k$ of characteristic 0 . Let $\partial \bar{X} \subset \bar{X}$ and $\partial \overline{X^{\prime}} \subset \overline{X^{\prime}}$ be reduced, normal crossing divisors such that $f^{-1}\left(\partial \overline{X^{\prime}}\right)_{\text {red }}$ is a normal crossing divisor in $\bar{X}$ which is contained in $\partial \bar{X}$.

Then there is a homomorphism of algebraic groups

$$
f_{*}: \operatorname{Pic}^{0}(\bar{X}, \partial \bar{X}) \rightarrow \operatorname{Pic}^{0}\left(\overline{X^{\prime}}, \partial \overline{X^{\prime}}\right)
$$

such that

(i) $f_{*}$ is compatible with the natural homomorphism $f_{*}: \operatorname{Pic}^{0}(\bar{X}) \rightarrow \operatorname{Pic}^{0}\left(\overline{X^{\prime}}\right)$ induced by the cycle theoretic direct image (i.e., push-forward) on divisors

(ii) the assignment $f \mapsto f_{*}$ is compatible with composition of appropriate proper maps

(iii) if $D$ is any divisor on $\bar{X}$ with support disjoint from $\partial \bar{X}$, and $[D] \in \operatorname{Pic}^{0}(\bar{X}, \partial \bar{X})$ is the class of the pair $\left(\mathcal{O}_{\bar{X}}(D), s_{D}\right)$ (where $s_{D}$ is the tautological meromorphic section of $\mathcal{O}_{\bar{X}}(D)$ with divisor $\left.D\right)$, then $f_{*}[D]=\left[f_{*} D\right] \in \operatorname{Pic}\left(\overline{X^{\prime}}, \partial \overline{X^{\prime}}\right)$, where $f_{*} D$ is the cycle theoretic direct image (i.e., push-forward) of $D$ under the proper map $f$, which is a divisor on $\overline{X^{\prime}}$ whose support is disjoint from $\partial \overline{X^{\prime}}$. 
Proof. - By considering the obvious map $\operatorname{Pic}^{0}(\bar{X}, \partial \bar{X}) \rightarrow \operatorname{Pic}^{0}\left(\bar{X}, f^{-1}\left(\partial \overline{X^{\prime}}\right)\right)$, we reduce immediately to the case when $\partial \bar{X}=f^{-1}\left(\partial \overline{X^{\prime}}\right)$. Now we can construct a Stein factorization diagram

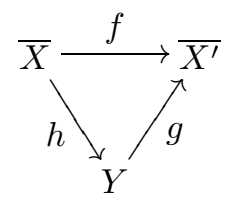

where $Y$ is a normal, proper variety of dimension $n, g: Y \rightarrow \overline{X^{\prime}}$ is a finite, surjective morphism, and $h$ is birational and proper with connected fibres. Further, $h_{*} \mathcal{O}_{\bar{X}}=\mathcal{O}_{Y}$, and $f_{*} \mathcal{O}_{\bar{X}}=g_{*} \mathcal{O}_{Y}$

Define $\partial Y=g^{-1}\left(\partial \overline{X^{\prime}}\right)_{\text {red }}$, so that $\partial \bar{X}=h^{-1}(\partial Y)_{\text {red }}$. Let $Z_{1}$ denote the union of the components of $Y_{\text {sing }}$ which are not contained in $\partial Y$. Let $Z^{\prime}=g\left(Z_{1}\right), Z=g^{-1}\left(Z^{\prime}\right)$, $\bar{Z}=h^{-1}\left(Z^{\prime}\right)$. Then $\bar{Z}, Z$ and $Z^{\prime}$ are each closed subsets of $\bar{X}, Y$ and $\overline{X^{\prime}}$, respectively, which have codimension $\geqslant 2$. Let $U=Y-Z, V=\bar{X}-\bar{Z}, W=\overline{X^{\prime}}-Z^{\prime}$, so that we have an induced commutative triangle of proper morphisms

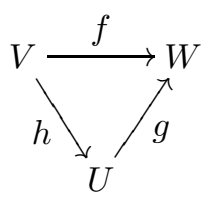

which is the Stein factorization of $f: V \rightarrow W$. Also define $\partial V=V \cap \partial \bar{X}, \partial U=Y \cap \partial Y$, $\partial W=W \cap \partial \overline{X^{\prime}}$

We now make the following claims.

(i) There is a homomorphism $\alpha: \operatorname{Pic}^{0}(\bar{X}, \partial \bar{X}) \rightarrow \operatorname{Pic}(U, \partial U)$, which fits into a commutative triangle

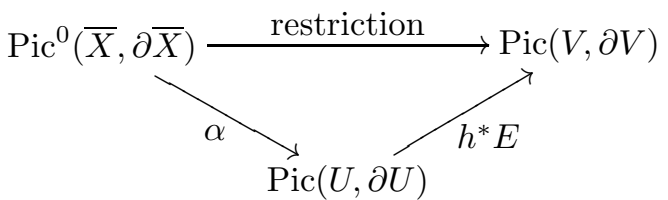

(ii) There is a norm map $g_{*}: \operatorname{Pic}(U, \partial U) \rightarrow \operatorname{Pic}(W, \partial W)$, such that (a) the composition $g_{*} \circ g^{*}$ is multiplication by $\operatorname{deg} g$, and (b) $g_{*}[D]=\left[g_{*} D\right]$ for the class of any Weil (= Cartier) divisor $D$ on $U$ with support disjoint from $\partial U$.

(iii) The natural restriction map $\rho: \operatorname{Pic}\left(\overline{X^{\prime}}, \partial \overline{X^{\prime}}\right) \rightarrow \operatorname{Pic}(W, \partial W)$ is an isomorphism.

Granting these claims, the desired map $f_{*}$ is the composition

$$
\operatorname{Pic}^{0}(\bar{X}, \partial X) \stackrel{\alpha}{\longrightarrow} \operatorname{Pic}(U, \partial U) \stackrel{g_{*}}{\longrightarrow} \operatorname{Pic}(W, \partial W) \stackrel{(\rho)^{-1}}{\longrightarrow} \operatorname{Pic}\left(\overline{X^{\prime}}, \partial \overline{X^{\prime}}\right) .
$$

This obviously factors through the subgroup $\operatorname{Pic}^{0}\left(\overline{X^{\prime}}, \partial \overline{X^{\prime}}\right)$, which is the maximal divisible subgroup of $\operatorname{Pic}\left(\overline{X^{\prime}}, \partial \overline{X^{\prime}}\right)$. 
We now proceed to prove the claims, in the order stated. First, we consider the $\operatorname{map} h^{*}: \operatorname{Pic}(U, \partial U) \rightarrow \operatorname{Pic}(V, \partial V)$. We have that

$$
\begin{aligned}
& \operatorname{Pic}(U, \partial U)=H^{1}\left(U, \mathcal{O}_{(U, \partial U)}^{*}\right), \\
& \operatorname{Pic}(V, \partial V)=H^{1}\left(V, \mathcal{O}_{(V, \partial V)}^{*}\right),
\end{aligned}
$$

where for a scheme $A$ and a closed subscheme $B$, we let $\mathcal{O}_{(A, B)}^{*} \stackrel{\text { def }}{=} \operatorname{ker}\left(\mathcal{O}_{A}^{*} \rightarrow \mathcal{O}_{B}^{*}\right)$. By the Leray spectral sequence for $h$, we obtain an exact sequence

$$
0 \rightarrow H^{1}\left(U, h_{*} \mathcal{O}_{(V, \partial V)}^{*}\right) \stackrel{h^{\prime}}{\longrightarrow} \operatorname{Pic}(V, \partial V) \rightarrow H^{0}\left(U, R^{1} h_{*} \mathcal{O}_{(V, \partial V)}^{*}\right),
$$

and $h^{*}: \operatorname{Pic}(U, \partial U) \rightarrow \operatorname{Pic}(V, \partial V)$ is the composition of $h^{\prime}$ with the natural map

$$
\operatorname{Pic}(U, \partial U)=H^{1}\left(U, \mathcal{O}_{(U, \partial U)}^{*}\right) \rightarrow H^{1}\left(U, h_{*} \mathcal{O}_{(V, \partial V)}^{*}\right) .
$$

In fact

$$
\mathcal{O}_{(U, \partial U)}^{*}=h_{*} \mathcal{O}_{(V, \partial V)}^{*},
$$

since $\mathcal{O}_{U}^{*}=h_{*} \mathcal{O}_{V}^{*}$, and the natural map $\mathcal{O}_{\partial U}^{*} \rightarrow h_{*} \mathcal{O}_{\partial V}^{*}$ is injective. This means we have an exact sequence

$$
0 \rightarrow \operatorname{Pic}(U, \partial U) \stackrel{h^{*}}{\longrightarrow} \operatorname{Pic}(V, \partial V) \rightarrow H^{0}\left(U, R^{1} h_{*} \mathcal{O}_{(V, \partial V)}^{*}\right) .
$$

So to construct the map $\alpha$ and the commutative triangle in Claim (i), it suffices to prove that the natural map

$$
\operatorname{Pic}^{0}(\bar{X}, \partial \bar{X}) \rightarrow H^{0}\left(U, R^{1} h_{*} \mathcal{O}_{(V, \partial V)}^{*}\right)
$$

vanishes.

Thus it suffices to prove that for each closed point $x \in U$, the map to the stalk at $x$

$$
\operatorname{Pic}^{0}(\bar{X}, \partial \bar{X}) \rightarrow\left(R^{1} h_{*} \mathcal{O}_{(V, \partial V)}^{*}\right)_{x}
$$

vanishes. We may identify $\left(R^{1} h_{*} \mathcal{O}_{(V, \partial V)}^{*}\right)_{x}$ with $H^{1}\left(V_{x}, \mathcal{O}_{\left(V_{x}, \partial V_{x}\right)}^{*}\right)$, where

$$
V_{x}=V \times_{U} \operatorname{Spec} \mathcal{O}_{x, U}, \quad \partial V_{x}=\partial V \times_{U} \operatorname{Spec} \mathcal{O}_{x, U} .
$$

So we want to show that the maps

$$
\operatorname{Pic}^{0}(\bar{X}, \partial \bar{X}) \rightarrow \operatorname{Pic}\left(V_{x}, \partial V_{x}\right)
$$

vanish, for all $x \in U$.

If $x \notin \partial U$, then $\partial V_{x}=\varnothing$. It suffices to see that the natural map

$$
\operatorname{Pic}^{0}(\bar{X}) \rightarrow \operatorname{Pic}\left(V_{x}\right)
$$

vanishes. Now $x$ is a non-singular point of $U$. Thus we can find a non-singular proper variety $\bar{U}$, containing $U_{\text {reg }}$ as a dense open subset; we can find a non-singular proper variety $\bar{V}$ containing $h^{-1}\left(U_{\text {reg }}\right)$ as a dense open set, and dominating $\bar{X}$. Then $\operatorname{Pic}^{0}(\bar{X}) \cong \operatorname{Pic}^{0}(\bar{V}) \cong \operatorname{Pic}^{0}(\bar{U})$, and evidently the map

$$
\operatorname{Pic}^{0}(\bar{U}) \rightarrow \operatorname{Pic}\left(V_{x}\right)
$$

vanishes, as it factors through $\operatorname{Pic}\left(\operatorname{Spec} \mathcal{O}_{x, U}\right)=0$. 
So we may take $x \in \partial U$. Now the fiber $h^{-1}(x)$ is contained in $\partial V$. Let $\widehat{\mathcal{O}}_{x, U}$ be the completion of $\mathcal{O}_{x, U}$, and let

$$
\widehat{V_{x}}=V \times_{U} \operatorname{Spec} \widehat{\mathcal{O}}_{x, U}, \quad \partial \widehat{V}_{x}=\partial V \times_{U} \operatorname{Spec} \widehat{\mathcal{O}}_{x, U}
$$

Then we have a natural homomorphism

$$
\operatorname{Pic}\left(V_{x}, \partial V_{x}\right) \rightarrow \operatorname{Pic}\left(\widehat{V_{x}}, \partial \widehat{V_{x}}\right)
$$

Since $\mathcal{O}_{x, U} \rightarrow \widehat{\mathcal{O}}_{x, U}$ is faithfully flat, we see easily that this homomorphism is injective. So we are reduced to proving that $\operatorname{Pic}^{0}(\bar{X}, \partial \bar{X}) \rightarrow \operatorname{Pic}\left(\widehat{V_{x}}, \partial \widehat{V_{x}}\right)$ vanishes.

For each $n \geqslant 1$, let $V_{x}^{n} \subset V_{x}$ be the closed subscheme defined by the $n$-th power of the ideal sheaf of the reduced fiber $h^{-1}(x)_{\text {red }}$. Let $\partial V_{x}^{n}$ denote the scheme theoretic intersection $\partial V \cap V_{x}^{n}$. Then $V_{x}^{1}=\partial V_{x}^{1}=h^{-1}(x)_{\text {red }}$, since $h^{-1}(x) \subset \partial V$. There is a natural homomorphism

$$
\operatorname{Pic}\left(\widehat{V_{x}}, \partial \widehat{V_{x}}\right) \rightarrow \underset{n}{\lim } \operatorname{Pic}\left(V_{x}^{n}, \partial V_{x}^{n}\right) \text {. }
$$

We claim that it is an isomorphism. This follows, using the five lemma, from the Grothendieck Existence Theorem [24], which gives isomorphisms

$$
\left.\operatorname{Pic}\left(\widehat{V_{x}}\right) \cong \lim _{n} \operatorname{Pic}\left(V_{x}^{n}\right), \operatorname{Pic}\left(\partial \widehat{V_{x}}\right) \cong{\underset{\lim }{n}}_{\operatorname{Pic}} \operatorname{Pi} V_{x}^{n}\right),
$$

and analogous isomorphisms on unit groups.

Hence we are reduced to proving that for each $n$, the natural restriction maps

$$
\operatorname{Pic}^{0}(\bar{X}, \partial \bar{X}) \rightarrow \operatorname{Pic}\left(V_{x}^{n}, \partial V_{x}^{n}\right)
$$

are zero. This is clear for $n=1$ since $V_{x}^{1}=\partial V_{x}^{1}$, so that $\operatorname{Pic}\left(V_{x}^{1}, \partial V_{x}^{1}\right)=0$. For $n>1$, one has that

$$
\operatorname{Pic}\left(V_{x}^{n}, \partial V_{x}^{n}\right)=\operatorname{ker}\left(\operatorname{Pic}\left(V_{x}^{n}, \partial V_{x}^{n}\right) \rightarrow \operatorname{Pic}\left(V_{x}^{1}, \partial V_{x}^{1}\right)\right)
$$

is an affine algebraic group which is purely of additive type (i.e., is a vector group) [10, Section 4]. Hence any homomorphism from a semi-abelian variety to $\operatorname{Pic}\left(V_{x}^{n}, \partial V_{x}^{n}\right)$ must vanish. This completes the proof of Claim (i).

Now we construct the norm map $g_{*}: \operatorname{Pic}(U, \partial U) \rightarrow \operatorname{Pic}(W, \partial W)$ of Claim (ii). First note that $R^{1} g_{*} \mathcal{O}_{(U, \partial U)}^{*}=0$, since the relative Picard group of a semi-local pair vanishes. Hence we have an identification

$$
\operatorname{Pic}(U, \partial U)=H^{1}\left(W, g_{*} \mathcal{O}_{(U, \partial U)}^{*}\right) .
$$

Since $U, W$ are integral and normal, and $g$ is finite surjective, the norm map on functions induces a homomorphism $N_{U / W}: g_{*} \mathcal{O}_{U}^{*} \rightarrow \mathcal{O}_{W}^{*}$. We claim this induces a map on subsheaves $N_{U / W}: g_{*} \mathcal{O}_{(U, \partial U)}^{*} \rightarrow \mathcal{O}_{(W, \partial W)}^{*}$, or equivalently, that the composition

$$
g_{*} \mathcal{O}_{(U, \partial U)}^{*} \hookrightarrow g_{*} \mathcal{O}_{U}^{*} \stackrel{N_{U / W}}{\longrightarrow} \mathcal{O}_{W}^{*} \rightarrow \mathcal{O}_{\partial W}^{*}
$$


vanishes. Since $\mathcal{O}_{\partial W}^{*}$ injects into the direct sum of constant sheaves associated to its stalks at the generic points of $\partial W$, it suffices to show that for any such generic point $\eta \in \partial W$, the map on stalks

$$
\left(g_{*} \mathcal{O}_{(U, \partial U)}^{*}\right)_{\eta} \rightarrow \mathcal{O}_{\eta, \partial W}^{*}
$$

vanishes. Now $\mathcal{O}_{\eta, \partial W}$ is the function field of an irreducible component of $\partial W$, and is the residue field of the discrete valuation ring $\mathcal{O}_{\eta, W}$. The stalk $\left(g_{*} \mathcal{O}_{U}^{*}\right)_{\eta}$ is the unit group of the (semi-local) integral closure of $\mathcal{O}_{\eta, W}$ in the function field of $U$; denote this semi-local ring by $\mathcal{O}_{\eta, U}$. The stalk $\left(g_{*} \mathcal{O}_{(U, \partial U)}^{*}\right)_{\eta}$ is the subgroup of $\mathcal{O}_{\eta, U}^{*}$ of units congruent to 1 modulo the Jacobson radical (which is the ideal defining $\partial U=g^{-1}(\partial W)_{\text {red }}$ in $\left.\mathcal{O}_{\eta, U}\right)$.

Now $\mathcal{O}_{\eta, U}$ is a free module over $\mathcal{O}_{\eta, W}$ of rank equal to the degree of $g$, and for any $a \in \mathcal{O}_{\eta, U}$, the norm of $a$ equals the determinant of the endomorphism of the free $\mathcal{O}_{\eta, W}$-module $\mathcal{O}_{\eta, U}$ given by multiplication by $a$. So it suffices to observe that if $a \in \mathcal{O}_{\eta, U}^{*}$ is congruent to 1 modulo the Jacobson radical, then this endomorphism is of the form $1+A$, where the matrix entries of $A$ lie in the maximal ideal of $\mathcal{O}_{\eta, W}$; hence the determinant of this matrix maps to 1 in the residue field of $\mathcal{O}_{\eta, W}$. This proves that

$$
\left(g_{*} \mathcal{O}_{(U, \partial U)}^{*}\right)_{\eta} \rightarrow \mathcal{O}_{\eta, \partial W}^{*}
$$

vanishes.

Now we define the map

$$
g_{*}: \operatorname{Pic}(U, \partial U) \rightarrow \operatorname{Pic}(W, \partial W)
$$

to be the map

induced by the sheaf map

$$
H^{1}\left(W, g_{*} \mathcal{O}_{(U, \partial U)}^{*}\right) \rightarrow H^{1}\left(W, \mathcal{O}_{(W, \partial W)}^{*}\right)
$$

$$
N_{U / W}: g_{*} \mathcal{O}_{(U, \partial U)}^{*} \rightarrow \mathcal{O}_{(W, \partial W)}^{*} .
$$

This evidently has the property that $g_{*} \circ g^{*}$ is multiplication by $\operatorname{deg} g$, since this is true at the sheaf level. To see the compatibility with the push-forward for divisors $D$ with support $|D|$ disjoint from $\partial U$, we compare the above map $g_{*}$ with the analogous map

$$
H_{g(|D|)}^{1}\left(W, g_{*} \mathcal{O}_{(U, \partial U)}^{*}\right) \rightarrow H_{g(|D|)}^{1}\left(W, \mathcal{O}_{(W, \partial W)}^{*}\right) .
$$

This completes the proof of Claim (ii).

To prove Claim (iii), it suffices to note that

$$
\operatorname{Pic}\left(\overline{X^{\prime}}\right) \cong \operatorname{Pic}(W), \operatorname{Pic}\left(\partial \overline{X^{\prime}}\right) \hookrightarrow \operatorname{Pic}(\partial W),
$$

and that

$$
H^{0}\left(\overline{X^{\prime}}, \mathcal{O}_{\overline{X^{\prime}}}^{*}\right) \cong H^{0}\left(W, \mathcal{O}_{W}^{*}\right), \quad H^{0}\left(\partial \overline{X^{\prime}}, \mathcal{O}_{\partial \overline{X^{\prime}}}^{*}\right) \cong H^{0}\left(\partial W, \mathcal{O}_{\partial W}^{*}\right) .
$$

All of these follow from the choice of the open set $W \subset \overline{X^{\prime}}$, such that $\overline{X^{\prime}}-W$ has codimension $\geqslant 2$ in $\overline{X^{\prime}}$, and $\partial \overline{X^{\prime}}-\partial W$ has codimension $\geqslant 2$ in $\partial \overline{X^{\prime}}$ (recall that $\overline{X^{\prime}}$ 
is integral, non-singular and complete, and $\partial \overline{X^{\prime}}$ is a reduced, normal crossing divisor in $\overline{X^{\prime}}$, and is hence a complete, equidimensional and Cohen-Macaulay scheme; thus $\overline{X^{\prime}}$ is locally connected in codimension 2 ).

Remark 6.2.3. - By making use of Propositions 6.2.1 and 1.3.1 we can see the following faithfulness property of the Albanese and Picard 1-motives. If we let $f$ : $X \rightarrow X^{\prime}$ be a generically finite morphism such that the push-forward $f_{*}: \mathrm{Pic}^{-}(X) \rightarrow$ $\mathrm{Pic}^{-}\left(X^{\prime}\right)$ induces an isomorphism on étale realizations then $f_{*}$ itself is an isomorphism of 1-motives. A similar statement holds for $f^{*}: \mathrm{Alb}^{+}\left(X^{\prime}\right) \rightarrow \operatorname{Alb}^{+}(X)$.

\subsection{Projective bundles and vector bundles}

Let $P=\mathbb{P}(\mathcal{E})=\operatorname{Proj} S(\mathcal{E})$ be the projective bundle associated to a locally free sheaf $\mathcal{E}$ on $X$ (here $S(\mathcal{E})$ is the symmetric algebra of $\mathcal{E}$ over $\mathcal{O}_{X}$ ).

Proposition 6.3.1. - There are canonical isomorphisms $\mathrm{Pic}^{-}(X) \cong \mathrm{Pic}^{-}(P)$ and $\operatorname{Pic}^{+}(X) \cong \operatorname{Pic}^{+}(P)$, therefore, dually, $\operatorname{Alb}^{+}(X) \cong \operatorname{Alb}^{+}(P)$ and $\operatorname{Alb}^{-}(X) \cong$ $\operatorname{Alb}^{-}(P)$.

Proof. - Let $\widetilde{P}=\mathbb{P}(\widetilde{\mathcal{E}}) \rightarrow \widetilde{X}$ be the pull-back along a chosen resolution of singularities $\widetilde{X} \rightarrow X$. We can choose a "Nash compactification" $\bar{X}$ of the resolution $\widetilde{X}$, i.e., we can also get a locally free sheaf $\overline{\mathcal{E}}$ on $\bar{X}$ which extends $\widetilde{\mathcal{E}}$ (to construct a Nash compactification, first choose an arbitrary one, and a coherent extension $\mathcal{F}$ of $\widetilde{\mathcal{E}}$; then resolve singularities of the Nash blow-up associated to $\mathcal{F}$, on which the pull-back of $\mathcal{F}$, modulo torsion, is a locally free sheaf).

We can then assume that $\widetilde{P}$ extends to $\bar{P}=\mathbb{P}(\overline{\mathcal{E}})$ on $\bar{X}$, and the boundary $\bar{P}-\widetilde{P}=Z$ is a normal crossing divisor in $\bar{P}$ which is a projective bundle over the normal crossing boundary $Y$ of $\bar{X}$. Since the Picard varieties of $\bar{X}$ and $\bar{P}$ are also isomorphic, the exact sequence (5) (cf. Proposition 2.1.2) yields an isomorphism of semi-abelian varieties $\operatorname{Pic}^{0}(\bar{X}, Y) \cong \operatorname{Pic}^{0}(\bar{P}, Z)$. Pull-back of divisors from $\bar{X}$ to $\bar{P}$ yields a compatible isomorphism between lattices, giving rise to the claimed isomorphism for $\mathrm{Pic}^{-}$; that for $\mathrm{Alb}^{+}$follows from Cartier duality.

For $\mathrm{Pic}^{+}$and $\mathrm{Alb}^{-}$, we argue as follows. Consider a Nash compactification $X^{*}$ of $X$, i.e., such that $\mathcal{E}$ extends to a locally free sheaf $\mathcal{E}^{*}$ on $X^{*}$. We can find a smooth proper hypercovering $\bar{X}$. of $X^{*}$ such that the induced reduced hypercovering of $X^{*}-X$ is a normal crossing divisor $Y$. in $\bar{X}$. Then $X .=\bar{X} .-Y$. yields a smooth proper hypercovering of $X$, and $\bar{X}$. is a smooth compactification with normal crossing boundary. Now $P .=P \times_{X} X$. is a smooth proper hypercovering of $P$, and we can get an induced compactification of $P$.

$$
\bar{P} .=\bar{X} \cdot \times_{X^{*}} \mathbb{P}\left(\mathcal{E}^{*}\right)
$$


which has normal crossing boundary. We then see easily that $\mathbf{P i c}^{0}(\bar{X}.) \cong \mathbf{P i c}(\bar{P}$. $)$ because of the exact sequence (31) ( $c f$. Proposition 4.1.2); similarly the lattices are isomorphic.

Let $V=\mathbb{V}(\mathcal{E})=\operatorname{Spec} S(\mathcal{E})$ be the geometric vector bundle associated to a locally free sheaf $\mathcal{E}$ on $X$. We have the following homotopy invariance property.

Proposition 6.3.2. - There is a canonical isomorphism $\operatorname{Pic}^{+}(X) \cong \operatorname{Pic}^{+}(V)$ and, dually, there is an isomorphism $\mathrm{Alb}^{-}(X) \cong \mathrm{Alb}^{-}(V)$.

Proof. - Consider a Nash compactification $X^{*}$ of $X$, so that $\mathcal{E}$ extends to a locally free sheaf $\mathcal{E}^{*}$ on $X^{*}$, and let $V^{*}=\mathbb{V}\left(\mathcal{E}^{*}\right)$. We let $\bar{X}$. be a smooth proper hypercovering of $X^{*}$ such that the reduced inverse image of $X^{*}-X$ is a normal crossing divisor, and let $V_{\text {. }}^{*}$ be the simplicial vector bundle on $\bar{X}$. obtained by the pull-back of $V^{*}$ along the hypercovering. We take

$$
\bar{V} \cdot=\bar{X} \cdot \times_{X^{*}} \mathbb{P}\left(\mathcal{E}^{*} \oplus \mathcal{O}_{X^{*}}\right)
$$

to be the compactification of $V^{*}$ with normal crossing boundary. We then have to show that

$$
\left[\operatorname{Div}_{Y \cdot}^{0}(\bar{X} \cdot) \rightarrow \operatorname{Pic}^{0}(\bar{X} \cdot)\right] \cong\left[\operatorname{Div}_{N}^{0}(\bar{V} \cdot) \rightarrow \operatorname{Pic}^{0}(\bar{V} \cdot)\right]
$$

where $N$. is the normal crossing boundary of $\bar{V}$., considered as a compactification of $V .=X . \times_{X} V$. We have

$$
N .=\bar{V} \cdot-V \cdot=Y \cdot \times_{X} \mathbb{P}\left(\mathcal{E}^{*} \oplus \mathcal{O}_{X^{*}}\right) \cup \bar{X} \cdot \times_{X^{*}} \mathbb{P}\left(\mathcal{E}^{*}\right) .
$$

Thus it is clear that the groups of divisors supported on $N$. and on $Y$, which are algebraically equivalent to zero (i.e., have classes in $\mathbf{P i c}{ }^{0}$ ) on the respective proper simplicial schemes, are naturally isomorphic; hence the lattices of our two 1-motives are naturally isomorphic. From the short exact sequence

$$
0 \rightarrow \operatorname{Pic}(\bar{X} \cdot) \rightarrow \operatorname{Pic}(\bar{V} \cdot) \rightarrow \mathbb{Z} \rightarrow 0
$$

we conclude that $\mathbf{P i c}{ }^{0}(\bar{X}$. $) \cong \mathbf{P i c}{ }^{0}(\bar{V}$. $)$, and we are done.

\subsection{Universality and zero-cycles}

We let $X$ be a projective $n$-dimensional $k$-variety. Let $X^{(n)}$ be the union of the $n$-dimensional irreducible components of $X$, and let $X_{\text {reg }}$ denote the locus of smooth points of $X$ which lie in $X^{(n)}$. We fix base points $x_{c} \in X^{c}$ in each component of $X_{\text {reg }}$, and let $a_{\mathbf{x}}^{+}: X_{\text {reg }} \rightarrow \operatorname{Alb}^{+}(X)$ be the corresponding Albanese map (see (29)). We denote by

$$
a_{X}^{+}: \mathcal{Z}^{n}\left(X_{\mathrm{reg}}\right)_{\operatorname{deg} 0} \rightarrow \mathrm{Alb}^{+}(X)
$$

the induced map on the group $\mathcal{Z}^{n}\left(X_{\text {reg }}\right)_{\operatorname{deg} 0}$ of zero cycles on $X_{\text {reg }}$ which have degree 0 on each component of $X_{\text {reg }}$; in fact $a_{X}^{+}$is independent of the choices of base points $\left\{x_{c}\right\}=\mathbf{x}$. 
We recall that the "cohomological" Levine-Weibel Chow group of zero-cycles $\mathrm{CH}^{n}(X)$ is defined to be the quotient of the free abelian group on (closed) points of $X_{\text {reg}}$, i.e., $\mathcal{Z}^{n}\left(X_{\text {reg }}\right)$, modulo the subgroup of zero-cycles which are divisors of appropriate rational functions on Cartier curves on $X(c f .[\mathbf{3 0}]$ and [8]).

Using $a_{X}^{+}$, we get a "motivic" construction of an Abel-Jacobi map, generalizing the Abel-Jacobi map for the Chow group of zero-cycles of degree 0 on projective nonsingular varieties, to the case of projective varieties with arbitrary singularities (this is done in $[\mathbf{2}]$ and $[\mathbf{8}]$ over $\mathbb{C}$ ). For a different algebraic construction, see [19].

TheOREM 6.4.1. - Let $X$ be a projective k-variety. The Albanese map $a_{X}^{+}$yields a universal regular homomorphism

$$
a^{+}: \mathrm{CH}^{n}(X)_{\operatorname{deg} 0} \rightarrow \mathrm{Alb}^{+}(X)
$$

from the "cohomological" Chow group of zero-cycles of degree zero to semi-abelian $k$-varieties.

We will prove the above theorem in several steps. We first construct Gysin maps for "good" curves, defined as follows. A curve $C \subset X$ is "good" if (i) $C$ is reduced, purely of dimension 1, and $C \cap S$ is reduced of dimension 0 (ii) $C$ is a local complete intersection in $X$ (iii) $C \subset X^{(n)}$ (iv) if $X_{n} \rightarrow X$ is the normalization, and we set $C_{0}=X_{n} \times_{X} C, S_{0}=X_{n} \times_{X}(C \cap S)$, then $C_{0}$ is also purely 1-dimensional, and $S_{0} \subset C_{0}$ consists of smooth points of $C_{0}$. Note that if $C$ is good, and $X^{<n}$ is the union of the irreducible components of $X$ of dimension $<n$, then $C \cap X^{<n}=\varnothing$.

Lemma 6.4.2. - Let $i: C \hookrightarrow X$ be a "good" curve in $X$. We then have Gysin maps

$$
i_{-}^{*}: \mathrm{Pic}^{-}(X) \rightarrow \mathrm{Pic}^{-}(C)
$$

and dually

$$
i_{*}^{+}: \operatorname{Alb}^{+}(C) \rightarrow \operatorname{Alb}^{+}(X) .
$$

Proof. - We may assume without loss of generality that $X$ is equidimensional. Let $X_{n} \rightarrow X$ be the normalization, $\widetilde{X} \rightarrow X_{n}$ a resolution of singularities, and $f: \widetilde{X} \rightarrow X$ the induced resolution of singularities. Since $C$ is "good", the scheme $C \times_{X} \widetilde{X}$ is a curve which is smooth at $f^{-1}(C \cap S)$.

Denote by $C^{\prime}$ the pull-back curve $\widetilde{X} \times_{X} C$. Let $f^{\prime}: C^{\prime} \rightarrow C$ be the restriction of $f$. Then $C^{\prime} \cong C_{0}=X_{n} \times_{X} C$, and the normalization $\widetilde{C} \rightarrow C$ of the curve $C$ clearly factors through $f^{\prime}: C^{\prime} \rightarrow C$. Let $\widetilde{i}: \widetilde{C} \rightarrow \widetilde{X}$ be the induced map. Then there is a natural pull-back map on Picard varieties $\widetilde{i}^{*}: \operatorname{Pic}^{0}(\widetilde{X}) \rightarrow \operatorname{Pic}^{0}(\widetilde{C})$. Thus, in order to get the claimed map $i_{-}^{*}$ on 1-motives, it is enough to show that any divisor $D \in \operatorname{Div} \underset{\widetilde{S} / S}{0}(\widetilde{X})$ pulls back to a divisor $\widetilde{i}^{*}(D) \in \operatorname{Div} \underset{\widetilde{C} / C}{0}(\widetilde{C})$. Since $C^{\prime}$ is smooth at the finite set of points $f^{-1}(C \cap S)$ it will suffices to show that $\left(i^{\prime}\right)^{*}(D) \in \operatorname{Div}_{C^{\prime} / C}^{0}\left(C^{\prime}\right)$ where $i^{\prime}: C^{\prime} \hookrightarrow \widetilde{X}$ is the canonical induced embedding. 
Now let $\widetilde{D}$ denote the support of $D$. Then $\widetilde{D}$ is mapped to $S$, and therefore $\widetilde{D} \times{ }_{X} C$, which is the support of $\left(i^{\prime}\right)^{*}(D)$, is mapped to $C \cap S$. We thus have the following diagram of Fulton's homological Chow groups

$$
\begin{array}{cccc}
\mathrm{CH}_{n-1}(\widetilde{D}) & \rightarrow \mathrm{CH}_{n-1}(S) & \rightarrow \mathrm{CH}_{n-1}(X) \\
\downarrow & & \downarrow \\
\mathrm{CH}_{0}\left(\widetilde{D} \times_{X} C\right) & \rightarrow \mathrm{CH}_{0}(C \cap S) & \hookrightarrow & \mathrm{CH}_{0}(C)
\end{array}
$$

by Fulton's compatibility result [21, Theorem 6.1] between pull-back and Gysin maps for locally complete intersection morphisms. Since the push-forward of $D$ vanishes as a cycle on $S$, the pull-back of $D$ to $\widetilde{D} \times{ }_{X} C$ pushes forward to zero in $\mathrm{CH}_{0}(C \cap S)$. Since $C \cap S$ is a reduced 0-dimensional scheme, the latter push-forward to $\mathrm{CH}_{0}(C \cap S)$ is in fact zero as a cycle on $C \cap S$.

We need the following compatibilities ( $c f$. Lemma 3.3-3.4 in [8]).

Lemma 6.4.3. - (a) Let $C$ be a "good" curve as in Lemma 6.4.2. There is a commutative diagram

$$
\begin{array}{cc}
\mathcal{Z}^{1}\left(C_{\mathrm{reg}}\right)_{\operatorname{deg} 0} \stackrel{a_{C}^{+}}{i_{*} \downarrow} & \mathrm{Alb}^{+}(C) \\
\mathcal{Z}^{n}\left(X_{\mathrm{reg}}\right)_{\operatorname{deg} 0} \stackrel{a_{X}^{+}}{\longrightarrow} & \operatorname{Alb}_{*}^{+}(X) .
\end{array}
$$

(b) Let $f: Y \rightarrow X$ be a morphism of $n$-dimensional projective varieties, such that $f_{\mid Y_{\text {reg }}}: Y_{\text {reg }} \rightarrow X_{\text {reg }}$ is a finite, flat morphism. Let $\gamma \in \mathcal{Z}^{n}\left(X_{\text {reg }}\right)_{\operatorname{deg} 0}$ be a zero-cycle of degree zero with inverse image $f^{*}(\gamma) \in \mathcal{Z}^{n}\left(Y_{\mathrm{reg}}\right)_{\operatorname{deg} 0}$. We then have

$$
a_{Y}^{+}\left(f^{*}(\gamma)\right)=f^{*}\left(a_{X}^{+}(\gamma)\right) .
$$

(c) If $f: Y \rightarrow X$ is a blow up at a smooth point of $X$ there is a commutative diagram

$$
\begin{aligned}
& \mathcal{Z}^{n}\left(Y_{\text {reg }}\right)_{\operatorname{deg} 0} \stackrel{a_{Y}^{+}}{\longrightarrow} \operatorname{Alb}^{+}(Y) \\
& f_{*} \downarrow \quad \cong \uparrow f^{*} \\
& \mathcal{Z}^{n}\left(X_{\mathrm{reg}}\right) \stackrel{a_{X}^{+}}{\longrightarrow} \mathrm{Alb}^{+}(X)
\end{aligned}
$$

The proof is left as an exercise for the reader.

Lemma 6.4.4. - Let $C$ be a reduced projective curve. The canonical section

$$
C_{\mathrm{reg}} \rightarrow \mathrm{Alb}^{+}(C)
$$

yields a universal regular homomorphism to semi-abelian $k$-varieties

$$
a^{+}: \mathrm{CH}^{1}(C)_{\operatorname{deg} 0} \rightarrow \mathrm{Alb}^{+}(C),
$$

which is an isomorphism when $C$ is semi-normal. 
Proof. - We recall that $\mathrm{CH}^{1}(C) \cong \operatorname{Pic}(C)$ and $\mathrm{CH}^{1}(C)_{\operatorname{deg} 0} \cong \operatorname{Pic}^{0}(C)$. Let $C^{\prime}$ be the semi-normalization of $C$; the canonical identification $\operatorname{Pic}^{0}\left(C^{\prime}\right) \cong \operatorname{Alb}^{+}(C)$ (see Proposition 3.1.2) together with the pull-back map $\operatorname{Pic}^{0}(C) \rightarrow \operatorname{Pic}^{0}\left(C^{\prime}\right)$, which is just the semi-abelian quotient of $\operatorname{Pic}^{0}(C)$, yields the result.

Now, in order to show that the map $a^{+}: \mathcal{Z}^{n}\left(X_{\text {reg }}\right)_{\operatorname{deg} 0} \rightarrow \operatorname{Alb}^{+}(X)(n>1)$ factors through rational equivalence, by $[\mathbf{8}]$, it suffices to show that ker $a^{+}$contains all divisors $(f)_{C}$ where: i) $C$ is a "good" curve in $X$, and ii) $f$ is a rational function on $C$ which is a unit at points of $C \cap S$. Using our Lemma 6.4.3 we adapt the proof of Lemma 3.5 in $[8]$ to our situation.

In order to show universality of $a^{+}: \mathrm{CH}^{n}(X)_{\operatorname{deg} 0} \rightarrow \mathrm{Alb}^{+}(X)$, we first note that, from the definitions, it is easy to see that $a^{+}$factors through the natural surjection $\mathrm{CH}^{n}(X)_{\operatorname{deg} 0} \rightarrow \mathrm{CH}^{n}\left(X^{(n)}\right)_{\operatorname{deg} 0}$, since by definition $\mathrm{Alb}^{+}(X)=\mathrm{Alb}^{+}\left(X^{(n)}\right)$. So we may assume $X$ is equidimensional.

Now consider the canonical extension

$$
0 \rightarrow T(S) \rightarrow \mathrm{Alb}^{-}\left(X_{\mathrm{reg}}\right) \rightarrow \mathrm{Alb}^{+}(X) \rightarrow 0
$$

(see Proposition 5.2.2). If $\psi: \mathrm{CH}^{n}(X)_{\operatorname{deg} 0} \rightarrow G$ is a regular homomorphism to a semi-abelian variety $G$, we need to find a unique factorisation

$$
\begin{array}{r}
\mathrm{CH}^{n}(X)_{\operatorname{deg} 0} \stackrel{a^{+}}{\longrightarrow} \mathrm{Alb}^{+}(X) \\
\\
\downarrow \\
\qquad \psi^{+} \\
G
\end{array}
$$

through $a^{+}$, for some homomorphism of algebraic groups $\psi^{+}$. Since $X_{\text {reg }}=\coprod_{c} U^{c}$ maps to $\mathrm{CH}^{n}(X)_{\operatorname{deg} 0}$ by taking a point $x \in U^{c}$ to the difference $x-x_{c}$ in the Chow group, we get a map $\psi_{0}: X_{\text {reg }} \rightarrow G$. By definition, since $\psi$ is a regular homomorphism, $\psi_{0}$ is a morphism, which sends each of the base points $x_{c}$ to 0 .

By the universal property (Proposition 5.2.1) of $\mathrm{Alb}^{-}\left(X_{\mathrm{reg}}\right), \psi_{0}$ factors through $\mathrm{Alb}^{-}\left(X_{\text {reg }}\right)$ yielding a map $\psi^{-}: \mathrm{Alb}^{-}\left(X_{\text {reg }}\right) \rightarrow G$.

Using the above-mentioned canonical extension, we need to show that $\psi^{-}(T(S))=$ 0 in order to obtain a well defined map $\psi^{+}$on the quotient semi-abelian variety $\mathrm{Alb}^{+}(X)$; the uniqueness of $\psi^{-}$will then imply that of $\psi^{+}$.

We have the following fact.

Lemma 6.4.5. - Let $i: C \hookrightarrow X$ be a complete intersection curve in $X$ which is "good" (i.e., satisfies the hypotheses of Lemma 6.4.2), such that $C$ meets every irreducible component of $S$, and moreover its singular locus $F$ is exactly $C \cap S$. We have a commutative diagram

$$
\begin{aligned}
& 0 \rightarrow T(S) \rightarrow \mathrm{Alb}^{-}\left(X_{\text {reg }}\right) \rightarrow \mathrm{Alb}^{+}(X) \rightarrow 0 \\
& \uparrow \quad \uparrow i_{*} \quad \uparrow i_{*}^{+} \\
& 0 \rightarrow T(F) \rightarrow \mathrm{Alb}^{-}\left(C_{\text {reg }}\right) \rightarrow \mathrm{Alb}^{+}(C) \rightarrow 0
\end{aligned}
$$

where $T(F) \rightarrow T(S)$ is a surjection of tori. 
Proof. - This follows easily from the dual statement, i.e., that the following diagram

$$
\begin{aligned}
& 0 \rightarrow \operatorname{Pic}^{-}(X) \rightarrow \operatorname{Pic}^{+}\left(X_{\text {reg }}\right) \rightarrow \operatorname{Div}_{S}^{0}(X) \rightarrow 0 \\
& 0 \rightarrow \operatorname{Pic}^{-}(C) \rightarrow \operatorname{Pic}^{+}\left(C_{\mathrm{reg}}\right) \rightarrow \operatorname{Div}_{F}^{0}(C) \rightarrow 0
\end{aligned}
$$

commutes. Moreover, $\operatorname{Div}_{S}(X)$ injects into $\operatorname{Div}_{F}(C)$.

By successive hyperplane sections we can always find a general complete intersection curve $C$ as above; therefore by Lemma 6.4.4 and Lemma 6.4.5 we conclude as follows. Since $i: C_{\text {reg }} \hookrightarrow X_{\text {reg }}$, we have that the composite of the following

$$
\mathrm{CH}^{1}(C)_{\operatorname{deg} 0} \stackrel{i_{*}}{\longrightarrow} \mathrm{CH}^{n}(X)_{\operatorname{deg} 0} \stackrel{\psi}{\longrightarrow} G
$$

yields a unique

$$
\psi_{C}^{+}: \operatorname{Alb}^{+}(C) \rightarrow G
$$

by the universal property for curves, i.e., Lemma 6.4 .4 ; whence $\psi_{C}^{+}(T(F))=0$, because the universal morphism $C_{\text {reg }} \rightarrow \mathrm{Alb}^{-}\left(C_{\text {reg }}\right)$ is compatible with $\psi_{C}^{+}$.

Since $T(F)$ surjects onto $T(S)$, the commutativity of the diagram in Lemma 6.4.5 implies that $\psi^{-}(T(S))=0$ as claimed. Thus Theorem 6.4.1 is proved.

\subsection{Gysin maps}

First consider the case of normal varieties.

Proposition 6.5.1. - Let $f: X^{\prime} \rightarrow X$ be any proper morphism of k-varieties, where $X$ is normal. We then have a functorial Gysin map

$$
f_{-}^{*}: \operatorname{Pic}^{-}(X) \rightarrow \operatorname{Pic}^{-}\left(X^{\prime}\right)
$$

and, dually,

$$
f_{*}^{+}: \operatorname{Alb}^{+}\left(X^{\prime}\right) \rightarrow \operatorname{Alb}^{+}(X)
$$

Proof. - Let $\bar{f}: \bar{X}^{\prime} \rightarrow \bar{X}$ be the induced map on smooth compactifications $\bar{X}^{\prime}$ and $\bar{X}$, compatibly with the normal crossing boundaries $Y^{\prime}$ and $Y$. We then have the following diagram of 1-motives

$$
\begin{gathered}
\operatorname{Pic}^{-}(X) \\
\| \downarrow \operatorname{Pic}^{-}\left(X^{\prime}\right) \\
\operatorname{Pic}^{-}(\widetilde{X}) \rightarrow \operatorname{Pic}^{-}\left(\widetilde{X}^{\prime}\right)
\end{gathered}
$$

yielding the claimed map, where since $X$ is normal we have that $\operatorname{Pic}^{-}(X) \cong$ $\operatorname{Pic}^{-}(\widetilde{X})=\operatorname{Pic}^{0}(\bar{X}, Y)$, and we have a pull-back map on relative line bundles

$$
\bar{f}^{*}: \operatorname{Pic}^{0}(\bar{X}, Y) \rightarrow \operatorname{Pic}^{0}\left(\bar{X}^{\prime}, Y^{\prime}\right) .
$$


We recall that a morphism $f: Z \rightarrow X$ is a projective local complete intersection morphism if can be factorized as $f=\pi \circ i$ for a regular embedding $i: Z \hookrightarrow P$ and a projection $\pi: P \rightarrow X$ from the projective bundle $P=\mathbb{P}(\mathcal{E})$ associated to a locally free $\mathcal{O}_{X}$-module $\mathcal{E}$.

Theorem 6.5.2. - Let $f: Z \rightarrow X$ be a projective local complete intersection morphism. We then get a functorial Gysin map

$$
f_{*}^{+}: \operatorname{Alb}^{+}(Z) \rightarrow \operatorname{Alb}^{+}(X)
$$

and, dually,

$$
f_{-}^{*}: \operatorname{Pic}^{-}(X) \rightarrow \operatorname{Pic}^{-}(Z)
$$

Proof. - Since $\mathrm{Alb}^{+}(P) \cong \mathrm{Alb}^{+}(X)$ by Proposition 6.3.1 we are left to prove our claim for regular imbeddings. We then have the following diagram

$$
\begin{aligned}
& \mathrm{CH}^{n}(Z)_{\operatorname{deg} 0} \stackrel{a_{Z}^{+}}{\longrightarrow} \mathrm{Alb}^{+}(Z) \\
& \quad i_{*} \downarrow \\
& \mathrm{CH}^{n}(P)_{\operatorname{deg} 0} \stackrel{a_{P}^{+}}{\longrightarrow} \mathrm{Alb}^{+}(P)
\end{aligned}
$$

where $i_{*}$ for cycles exists trivially, and therefore, by Theorem 6.4.1, the composite of $i_{*}$ and $a_{P}^{+}$factors through $\mathrm{Alb}^{+}(Z)$.

In order to show that the construction is independent of the factorisation, we observe that it is so on the étale realizations (where it coincides with the Gysin map obtained via Grothendieck-Verdier duality), and therefore, by Proposition 1.3.1, we are done.

REMARK 6.5.3. - It would of course be of interest to have the same result for proper local complete intersection morphisms as well, for which the above strategy of comparison with the Levine-Weibel Chow group of 0-cycles is not applicable.

It would also be desirable to have a "geometric" proof of independence of the Gysin map from the choice of factorization, instead of the above one using the étale realization. 

CHAPTER 7

\section{RATIONALITY QUESTIONS}

We now consider the above theory in the case when the ground field $k$ is an arbitrary field of characteristic 0 . Let $\bar{k}$ denote a fixed algebraic closure of $k$; if $A$ is any "object" (1-motive, scheme, morphism, sheaf ...) over $k$, then $A_{\bar{k}}$ will denote its base change to $\bar{k}$.

\subsection{1-motives over non-closed fields}

Consider a 1-motive $M=[L \stackrel{u}{\rightarrow} G]$ over $k$. By definition, this is a homomorphism between $k$-group schemes, where $L$ is an étale group scheme, and $G$ a semi-abelian scheme, such that $L_{\bar{k}}$ is a lattice (free abelian group of finite rank). The lattice $L_{\bar{k}}$ is naturally a module over the Galois group $\operatorname{Gal}(\bar{k} / k)$, and the étale group scheme $L$ is determined by this Galois module. The Galois group operates semi-linearly on $G_{\bar{k}}=G \times_{k} \bar{k}$ as well, and the morphism $u$ is determined uniquely by the morphism $u_{\bar{k}}: L_{\bar{k}} \rightarrow G_{\bar{k}}$, which is $\operatorname{Gal}(\bar{k} / k)$-equivariant. Conversely, any Galois equivariant morphism $L_{\bar{k}} \rightarrow G_{\bar{k}}$ is necessarily of the form $u_{\bar{k}}$.

Thus, to give a 1-motive over $k$ is to give (i) a semi-abelian $k$-scheme $G$ (ii) a lattice $\bar{L}$ which underlies a $\operatorname{Gal}(\bar{k} / k)$-module (iii) a 1-motive $\left[\bar{L} \stackrel{\bar{u}}{\rightarrow} G_{\bar{k}}\right]$ over $\bar{k}$, such that $\bar{u}$ is $\operatorname{Gal}(\bar{k} / k)$-equivariant, for the given module structure on $\bar{L}$, and the natural semi-linear action on $G_{\bar{k}}$.

If $k \hookrightarrow \mathbb{C}$, then for any 1-motive $M$ over $k$, we obtain a corresponding 1-motive $M_{\mathbb{C}}$ over $\mathbb{C}$, which has a Hodge realization. For the étale realization, note that $\widehat{T}\left(M_{\bar{k}}\right)$ is a free $\widehat{\mathbb{Z}}$-module of finite rank, which supports a natural action of $\mathrm{Gal}(\bar{k} / k)$. We call this Galois module the étale realization of $M$. Finally, if $M^{\natural}$ denotes the universal $\mathbb{G}_{a}$-extension of $M$ in the category of complexes of $k$-group schemes, then $M_{\frac{\natural}{k}}^{\natural}$ is the universal $\mathbb{G}_{a}$-extension of $M_{\bar{k}}$ in the category of complexes of $\bar{k}$-group schemes, and

$$
\operatorname{Lie}\left(M^{\natural}\right)_{\bar{k}} \cong \operatorname{Lie}\left(M_{\frac{k}{k}}^{\natural}\right)
$$


as filtered $\bar{k}$-vector spaces. We define the De Rham realization $T_{\mathrm{DR}}(M)$ to be the filtered $k$-vector space $\operatorname{Lie}\left(M^{\natural}\right)$.

\subsection{Albanese and Picard 1-motives in zero characteristic}

The aim of this section is to show that if $X$ is a $k$-variety, then there are naturally defined 1-motives $\mathrm{Pic}^{+}(X), \mathrm{Pic}^{-}(X), \mathrm{Alb}^{+}(X), \mathrm{Alb}^{-}(X)$ defined over $k$, pairwise Cartier dual, with the following properties.

(i) If $k^{\prime}$ is an extension field of $k$, the corresponding 1-motives for $X_{k^{\prime}}$ are obtained by base change from $k$ to $k^{\prime}$ from the 1-motives for $X$.

(ii) The étale realizations coincide with appropriate the étale (co)homology groups (modulo torsion) of $X_{\bar{k}}$ as $\operatorname{Gal}(\bar{k} / k)$-modules, where the Galois action on étale (co)homology is the standard one.

(iii) The De Rham realizations coincide, as filtered $k$-vector spaces, with the appropriate De Rham (co)homology groups of $X$ (defined as in (2.6.3) via suitable hypercoverings and compactifications over $k$ ).

The proofs of the above assertions are fairly straightforward, and basically amount to the observation that, when we carry out the constructions of 1-motives for $X_{\bar{k}}$ as in the earlier sections, and consider the computations of realizations, these are sufficiently natural as to be automatically compatible with the action of $\operatorname{Gal}(\bar{k} / k)$. As such, our arguments will be a little sketchy.

First consider the construction of $\mathrm{Pic}^{-}(X)$. Let $n=\operatorname{dim} X=\operatorname{dim} X_{\bar{k}}$. If $X_{\bar{k}}^{(n)}$ is the union of the $n$-dimensional irreducible components of $X_{\bar{k}}$, then it corresponds to a unique closed $k$-subscheme $X^{(n)}$ of $X$, which is also purely of dimension $n$. So we reduce to the case when $X$ and $X_{\bar{k}}$ are equidimensional.

Now we may choose a resolution of singularities $f: \widetilde{X} \rightarrow X$, and a compactification $\bar{X}$ of $\tilde{X}$, both defined over $k$, such that $\bar{X}_{\bar{k}}$ is a good normal crossing compactification of the resolution $\tilde{X}_{\bar{k}} \rightarrow X_{\bar{k}}$. Let $Y \subset \bar{X}$ be the normal crossing boundary divisor, $S \subset X$ the singular locus, $\bar{S} \subset \bar{X}$ the Zariski closure of $f^{-1}(S)$.

Lemma 2.1.1 gives the representability of the relative Picard functor of the pair $(\bar{X}, Y)$ by a $k$-group scheme $(\operatorname{say}, \operatorname{Pic}(\bar{X}, Y))$, locally of finite type, whose $\bar{k}$-points coincide with the relative Picard group $\operatorname{Pic}\left(\bar{X}_{\bar{k}}, Y_{\bar{k}}\right)$.

The identity component $\operatorname{Pic}^{0}\left(\bar{X}_{\bar{k}}, Y_{\bar{k}}\right)$ is stable under the semi-linear $\operatorname{Gal}(\bar{k} / k)$ action on $\operatorname{Pic}(\bar{X}, Y)_{\bar{k}}$, and so naturally determines a $k$-subgroup $\operatorname{scheme~}_{\operatorname{Pic}}^{0}(\bar{X}, Y)$ of $\operatorname{Pic}(\bar{X}, Y)$. The lattice $\operatorname{Div} \bar{S}_{\bar{k}} / S_{\bar{k}}\left(\bar{X}_{\bar{k}}, Y_{\bar{k}}\right)$ is evidently stable under $\operatorname{Gal}(\bar{k} / k)$, with respect to the natural Galois action on Weil divisors on $\bar{X}_{\bar{k}}$.

Finally, the canonical map

$$
\operatorname{Div}{\frac{0}{S_{\bar{k}}} / S_{\bar{k}}}\left(\bar{X}_{\bar{k}}, Y_{\bar{k}}\right) \rightarrow \operatorname{Pic}^{0}\left(\bar{X}_{\bar{k}}, Y_{\bar{k}}\right)
$$


is clearly Galois equivariant. Hence we obtain a well-defined 1-motive over $k$, which we define to be $\mathrm{Pic}^{-}(X)$; by construction we then have $\mathrm{Pic}^{-}\left(X_{\bar{k}}\right)=\mathrm{Pic}^{-}(X)_{\bar{k}}$.

The isomorphism

$$
\widehat{T}\left(\operatorname{Pic}^{-}\left(X_{\bar{k}}\right)\right) \rightarrow H_{2 n-1}^{\text {ét }}\left(X_{\bar{k}}, \widehat{\mathbb{Z}}(1-n)\right) /(\text { torsion })
$$

is $\operatorname{Gal}(\bar{k} / k)$-equivariant, since it ultimately rests on the identification, via Kummer theory, of étale $\mu_{m}$-coverings of certain open subschemes of $X_{\bar{k}}$ with isomorphism classes of triples $(\mathcal{L}, \varphi, \alpha)$ (see Proposition 2.5.2) consisting of $m$-torsion line bundles $\mathcal{L}$ with additional trivializing data; but this identification is easily seen to be Galois equivariant, where the Galois group operates on such triples in the obvious way (corresponding to the natural Galois action on $T_{\mathbb{Z} / m}\left(\mathrm{Pic}^{-}\left(X_{\bar{k}}\right)\right)$ ), while it acts on the collection of étale coverings by twisting (changing the structure morphism to Spec $\bar{k}$ ), which corresponds to the natural action on étale (co)homology.

As for the De Rham realization, the same proof that

$$
T_{\mathrm{DR}}\left(\operatorname{Pic}^{-}\left(X_{\bar{k}}\right)\right)=\operatorname{Lie}\left(\operatorname{Pic}^{-}\left(X_{\bar{k}}\right)^{\natural}\right)
$$

coincides, as a filtered $\bar{k}$-vector space with $H_{2 n-1}^{D R}\left(X_{\bar{k}}\right)(1-n)$, yields a proof that $H_{2 n-1}^{D R}(X)(1-n)$ coincides with $\mathrm{Lie}\left(\mathrm{Pic}^{-}(X)^{\natural}\right)$ as a filtered $k$-vector space, provided we have 1 fact: that for $Z \subset \bar{X}$ as in Lemma 2.6.2, the Lie algebra computation in Lemma 2.6.2(d) is valid.

This is of course clear over $\bar{k}$, from the formula in Lemma 2.6.2(a)

$$
\operatorname{Pic}^{\natural-\log }\left(\bar{X}_{\bar{k}}-Z_{\bar{k}}, Y_{\bar{k}}\right) \cong \mathbb{H}^{1}\left(\bar{X}_{\bar{k}}, \mathcal{O}_{\bar{X}_{\bar{k}}, Y_{\bar{k}}}^{*} \stackrel{\mathrm{dlog}}{\longrightarrow} \Omega_{\bar{X}_{\bar{k}}}^{1}\left(\log \left(Y_{\bar{k}}+Z_{\bar{k}}\right)\right)\left(-Y_{\bar{k}}\right)\right) \text {. }
$$

The analogous formula may not be valid over $k$, since the expression on the right side arises as the value (on $k$, or $\bar{k}$ ) of an appropriate Picard functor, while the left side refers to the sections of the associated fpqc sheaf (these do coincide over $\bar{k}$, while this is unclear over $k$ ).

But the tangent space at the identity to the Picard functor admits a $k$-linear transformation to the corresponding tangent space of the representable functor given by the associated $f p q c$-sheaf. This linear transformation, upon base change to $\bar{k}$, is an isomorphism of (filtered) vector spaces. Hence it is an isomorphism over $k$ as well. So the "presheaf tangent space" is the same as the true tangent space (this applies also to the simplicial Picard functor).

The results for $\mathrm{Alb}^{+}(X)$ now follow by Cartier duality from those for $\mathrm{Pic}^{-}(X)$.

Next, consider $\mathrm{Pic}^{+}(X)$. We can choose a smooth proper hypercovering $X . \rightarrow X$ and a smooth compactification $\bar{X}$. of $X$. with normal crossing boundary $Y$., all in the category of simplicial $k$-schemes.

The $f p q c$-sheaf associated to the simplicial Picard functor of $\bar{X}$. is representable by a $k$-group scheme, locally of finite type, whose identity component is a semi-abelian $k$-scheme $\mathbf{P i c}^{0}(\bar{X} \cdot)$, such that

$$
\operatorname{Pic}^{0}(\bar{X} \cdot)_{\bar{k}}=\operatorname{Pic}^{0}\left((\bar{X} \cdot)_{\bar{k}}\right) .
$$


The lattice $\operatorname{Div}_{\left(Y_{\bullet}\right)_{\bar{k}}}^{0}\left((\bar{X} \cdot)_{\bar{k}}\right)$ is a Galois module in an obvious way, such that the map defining the 1-motive $\operatorname{Pic}^{+}\left(X_{\bar{k}}\right)$ is Galois equivariant.

Thus there is a well-defined 1-motive $\operatorname{Pic}^{+}(X)$, defined over $k$, such that there is an identification $\operatorname{Pic}^{+}(X)_{\bar{k}}=\operatorname{Pic}^{+}\left(X_{\bar{k}}\right)$. The discussion of the properties of the étale and De Rham realizations is very similar to that of $\mathrm{Pic}^{-}(X)$, and is left to the reader. The case of $\mathrm{Alb}^{-}(X)$ follows by Cartier duality.

\subsection{Albanese mappings}

From the above constructions of 1-motives over $k$, it is also clear that various functorial properties, and Albanese mappings, are all defined over $k$ as well, possibly after slight reformulation. For example, if $f: X \rightarrow X^{\prime}$ is a morphism between $n$ dimensional $k$-varieties, then there is a push-forward $f_{*}: \mathrm{Pic}^{-}(X) \rightarrow \mathrm{Pic}^{-}\left(X^{\prime}\right)$, and a pull-back $f^{*}: \mathrm{Alb}^{+}\left(X^{\prime}\right) \rightarrow \mathrm{Alb}^{+}(X)$. For an arbitrary morphism of $k$-varieties, there is a pull-back $f^{*}: \mathrm{Pic}^{+}\left(X^{\prime}\right) \rightarrow \mathrm{Pic}^{+}(X)$ and a push-forward $\mathrm{Alb}^{-}(X) \rightarrow \mathrm{Alb}^{-}\left(X^{\prime}\right)$.

For the Albanese mappings $a_{\mathbf{x}}^{+}: X_{\text {reg }} \rightarrow \operatorname{Alb}^{+}(X)$ and $a_{\mathbf{x}}^{-}: X_{\text {reg }} \rightarrow \mathrm{Alb}^{-}\left(X_{\text {reg }}\right)$, these exist over $k$ provided we can choose the base points $x_{c} \in X^{c} \cap\left(X_{\text {reg }}\right)_{\bar{k}}$ to be $k$-rational points, or more generally, if the 0 -cycle $\sum_{c} x_{c}$ is defined over $k$ (i.e., is $\operatorname{Gal}(\bar{k} / k)$-invariant). However, we cannot in general choose such base-points. Instead, we can consider the map

$$
\left(A^{-}\right)_{\bar{k}}: \coprod_{c} X_{c} \times X_{c} \subset\left(X_{\mathrm{reg}} \times X_{\mathrm{reg}}\right)_{\bar{k}} \rightarrow \operatorname{Alb}^{-}\left(X_{\mathrm{reg}}\right)_{\bar{k}}
$$

given by $\left(A^{-}\right)_{\bar{k}}(x, y)=a_{\mathbf{x}}^{-}(x)-a_{\mathbf{x}}^{-}(y)$, which is in fact independent of $\mathbf{x}=\left\{x_{c}\right\}_{c}$, and $\operatorname{Gal}(\bar{k} / k)$-equivariant, thus yielding a map of $k$-varieties

$$
A^{-}: U \rightarrow \mathrm{Alb}^{-}\left(X_{\mathrm{reg}}\right),
$$

where $U \subset X_{\text {reg }} \times X_{\text {reg }}$ is the open $k$-subscheme consisting of the union of the connected components intersecting the diagonal, and so $U_{\bar{k}}=\bigsqcup_{c} X_{c} \times X_{c}$.

The morphism $A^{-}$is universal among those $k$-morphisms from $U$ to semi-abelian $k$-varieties such that the natural involution on $U$ intertwines with multiplication by -1 on the semi-abelian variety.

In a similar way, we can define a Galois equivariant $\bar{k}$-morphism

$$
\left(A^{+}\right)_{\bar{k}}: \coprod_{c} X_{c} \times X_{c} \subset\left(X_{\mathrm{reg}} \times X_{\mathrm{reg}}\right)_{\bar{k}} \rightarrow \operatorname{Alb}^{+}(X)_{\bar{k}},
$$

and hence a $k$-morphism

$$
A^{+}: U \rightarrow \operatorname{Alb}^{+}(X)
$$




\section{APPENDIX}

\section{PICARD FUNCTORS}

Let $\mathcal{S} c h_{k}$ be the category of schemes over a field $k$. We will consider contravariant functors from $\mathcal{S} c h_{k}$ to $\mathcal{A} b$, the category of abelian groups; we will refer to such a functor as a presheaf on $\mathcal{S} c h_{k}$. We are interested in representing such functors, when possible, by $k$-group schemes of finite type whose identity component is a semi-abelian $k$-scheme, i.e., an extension of an abelian scheme by a $k$-torus.

\section{A.1. Axioms}

We recall that according to Grothendieck [23] and Murre [36] a presheaf $F$ is representable by a $k$-group scheme, locally of finite type, if and only if a certain list of 7 axioms is satisfied. This implies the following necessary and sufficient conditions for representability by a group scheme whose identity component is semi-abelian, where $\mathrm{P}^{\prime}$ is a modification of $[\mathbf{3 6}, \mathrm{P} 7]$.

P1 : $F$ is strictly pro-representable and the local components at rational points are noetherian;

P2 : if $A=\lim _{n} A / \wp^{n+1}$ is a local $k$-algebra which is complete and separated w.r.t. the $\wp$-adique topology, then $F(A) \cong \lim _{n} F\left(A / \wp^{n+1}\right)$;

P3 : if $A=\lim _{\alpha} A_{\alpha}$ as $k$-algebras, then $F(A) \cong \lim _{\alpha} F\left(A_{\alpha}\right)$;

P4-P5 : $F$ is a fpqc-sheaf;

P6 : if $T \in \mathcal{S} c h_{k}$ and $\xi$ is a $T$-point of $F$, then $N(\xi) \stackrel{\text { def }}{=}\left\{f: T^{\prime} \rightarrow T / F(f)(\xi)=0\right\}$ yields a closed subscheme of $T$;

$\mathbf{P 7}^{\prime}$ : if $\xi$ is a $V$-point of $F$, for $V=C-S$ a Zariski open of a projective nonsingular $\bar{k}$-curve $C$, then the induced map of $\bar{k}$-points has the module $\mathfrak{m}=S$, in the sense of Serre [49].

Here, in $\mathrm{P}^{\prime}$, the condition on $\mathfrak{m}$ is that if $S=\left\{P_{1}, \ldots, P_{r}\right\}$, then the non-negative integers $n_{1}, \ldots, n_{r}$ involved in the definition of a modulus (see [49, page 10]) are all taken to be 1 .

Let $F, F^{\prime}$ and $F^{\prime \prime}$ be presheaves on $\mathcal{S} c h_{k}$ forming an extension

$$
0 \rightarrow F^{\prime} \rightarrow F \rightarrow F^{\prime \prime} \rightarrow 0
$$


i.e., such that $0 \rightarrow F^{\prime}(T) \rightarrow F(T) \rightarrow F^{\prime \prime}(T) \rightarrow 0$ is an exact sequence of abelian groups for any $T \in \mathcal{S} c h_{k}$, which is natural in $T$. Denote by

$$
0 \rightarrow \widetilde{F}^{\prime} \rightarrow \widetilde{F} \rightarrow \widetilde{F}^{\prime \prime} \rightarrow 0
$$

the exact sequence of associated sheaves for the fpqc-topology (i.e., the faithfully flat and quasi-compact topology). If we let

$$
Q(T) \stackrel{\text { def }}{=} \operatorname{coker}\left(\widetilde{F}(T) \rightarrow \widetilde{F}^{\prime \prime}(T)\right)
$$

for $T \in \mathcal{S} c h_{k}$, then $Q$ is a functor on $\mathcal{S} c h_{k}$.

Lemma A.1.1. - Assume that the fpqc-sheaves $\widetilde{F}^{\prime}$ and $\widetilde{F}^{\prime \prime}$ satisfy the axioms P1-P3 and $\mathrm{P} 6$. Further assume that i) $Q$ vanishes on the subcategory of of artinian algebras, ii) $Q$ vanishes on the subcategory of local complete algebras, and iii) $\lim _{\alpha} Q\left(A_{\alpha}\right)$ injects into $Q\left(\lim _{\alpha} A_{\alpha}\right)$.

Then the fpqc-sheaf $\widetilde{F}$ satisfies the axioms $\mathrm{P} 1-\mathrm{P} 3$ and $\mathrm{P} 6$.

Proof. - The following sequence

$$
0 \rightarrow \widetilde{F}^{\prime}(T) \rightarrow \widetilde{F}(T) \rightarrow \widetilde{F}^{\prime \prime}(T) \rightarrow Q(T) \rightarrow 0
$$

is exact and natural in $T \in \mathcal{S} c h_{k}$. To show pro-representability we use Grothendieck's criterion in $[\mathbf{2 3}, 195-5 / 9]$, saying that $\widetilde{F}$ needs to be left exact on the subcategory of artinian algebras. From $i$ ) and (42) one can see that $\widetilde{F}$ is pro-representable. Then there is a topological algebra $\mathcal{O}$ such that $\widetilde{F}(A) \cong \operatorname{Hom}_{c}(\mathcal{O}, A)$.

We show that the local components are noetherian by using Grothendieck's criterion [23, Prop.5.1, 195-8]. In fact, the local component at a point $\xi$ is pro-represented by the localization $\mathcal{O}_{\wp \xi}$, where $\wp_{\xi}=\operatorname{ker}(\mathcal{O} \stackrel{\xi}{\rightarrow} k)$, and in order to show that $\mathcal{O}_{\wp \xi}$ is noetherian it will suffices to show that $\left(\wp_{\xi} / \wp_{\xi}^{2}\right)^{\vee}$ is finite dimensional. For any $k$-scheme $T$ the $k$-point $\xi$ of $\widetilde{F}$ induces an element $\xi_{T} \in \widetilde{F}(T)$ by pulling back along the structural morphism; we then get an automorphism $(+\xi)_{*}: \widetilde{F} \cong \widetilde{F}$ by adding $\xi_{T}$ in $F(T)$. Thus we can assume $\xi=0$, therefore we have

$$
\left(\wp_{\xi} / \wp_{\xi}^{2}\right)^{\vee}=\operatorname{ker}\left(\widetilde{F}\left(k[t] /\left(t^{2}\right)\right) \rightarrow \widetilde{F}(k)\right) .
$$

By (42) we conclude that P1 is satisfied.

Axioms P2-P3 follow from a diagram chase, because of (42) and the assumptions ii)-iii). To show $\mathrm{P} 6$ we proceed as follows. Let $\xi: T \rightarrow \widetilde{F}$ be a point, i.e., $\xi \in \widetilde{F}(T)$. We have to show that $N(\xi)$ is a closed subscheme of $T$. Let $\xi^{\prime \prime} \in \widetilde{F}^{\prime \prime}(T)$ be the induced point of $\widetilde{F}^{\prime \prime}$ and let $i: N\left(\xi^{\prime \prime}\right) \hookrightarrow T$ be the closed embedding. Then $\widetilde{F}(i)(\xi)$ actually belongs to $\widetilde{F}^{\prime}\left(N\left(\xi^{\prime \prime}\right)\right)$ since it yields zero in $\widetilde{F}^{\prime \prime}\left(N\left(\xi^{\prime \prime}\right)\right)$. Then $N(\widetilde{F}(i)(\xi))$ is a closed subscheme of $N\left(\xi^{\prime \prime}\right)$ hence of $T$. We can see that $N(\xi)=N(\widetilde{F}(i)(\xi))$. In fact, if $\alpha: T^{\prime} \rightarrow N\left(\xi^{\prime \prime}\right)$ is a point such that $\widetilde{F}(\alpha) \widetilde{F}(i)(\xi)=0$ then $i \alpha: T^{\prime} \rightarrow T$ belongs to $N(\xi)$. Conversely, if $\alpha: T^{\prime} \rightarrow T$ is such that $\widetilde{F}(\alpha)(\xi)=0$ then $\alpha$ belongs to $N\left(\xi^{\prime \prime}\right)$ as well which means that $\alpha=i \beta$ where $\beta: T^{\prime} \rightarrow N\left(\xi^{\prime \prime}\right)$ whence $\beta \in N(\widetilde{F}(i)(\xi))$. 
If $F$ is any functor from $\mathcal{S} c h_{k}^{o p}$ to abelian groups, and if $x \in X$ is a point, let $x^{*}: F(X) \rightarrow F(k(x))$ denote the homomorphism induced by the inclusion morphism $\operatorname{Spec} k(x) \rightarrow X$ determined by $x$.

Lemma A.1.2. - Let $k$ be an algebraically closed field, and let $F: \mathcal{S} c h_{k}^{o p} \rightarrow \mathcal{A} b$ be a functor, satisfying:

(a) the natural map $F(k) \rightarrow F\left(\mathbb{P}^{1}-\{1\}\right)$ (induced by the structure morphism) is surjective, and

(b) if $V$ is a non-singular quasi-projective $k$-curve, the image of the natural map $F\left(S^{n} V\right) \rightarrow F\left(V^{n}\right)$ is the subgroup of invariants under the natural action of the permutation group.

Then for any non-singular projective $k$-curve, any finite subset $S \subset C(k)$, and any rational function $f \in k(C)$ which is regular on $S$ and has $f(x)=1$ for all $x \in S$, we have that

$$
\sum_{x \in C(k)} \operatorname{ord}_{x}(f) x^{*}: F(C-S) \rightarrow F(k)
$$

is the zero map. Thus F satisfies condition $\mathrm{P} 7$ ' above.

Proof. - Let $C$ be a non-singular projective $k$-curve, $S \subset C(k)$ a finite set of closed points. For any divisor $\delta=\sum_{i} n_{i} x_{i}$ on $C-S$, let $\delta^{*}: F(C-S) \rightarrow F(k)$ be the map given by $\delta^{*}=\sum_{i} n_{i} x_{i}^{*}$. Clearly $\delta \mapsto \delta^{*}$ is a homomorphism from divisors on $C_{S}$ to $\operatorname{Hom}(F(C-S), F(k))$. If $\delta$ is an effective divisor of degree $n$, then $\delta$ determines a point $[\delta] \in S^{n}(C-S)$ in an obvious way, and hence a homomorphism

$$
[\delta]^{*}: F\left(S^{n}(C-S)\right) \rightarrow F(k) .
$$

There is a homomorphism

$$
\left(\sum_{i=1}^{n} \pi_{i}^{*}\right): F(C-S) \rightarrow F\left((C-S)^{n}\right),
$$

where $\pi_{i}:(C-S)^{n} \rightarrow C-S$ is the $i^{\text {th }}$ projection. Clearly the image is contained in the subgroup of invariants for the action of the permutation group $S_{n}$. Hence there exists a map of sets (not necessarily unique, or even a homomorphism)

$$
\psi: F(C-S) \rightarrow F\left(S^{n}(C-S)\right), \quad \xi \mapsto S^{n}(\xi)
$$

such that $S^{n}(\xi)$ is a pre-image in $F\left(S^{n}(C-S)\right.$ of $\left(\sum_{i=1}^{n} \pi_{i}^{*}\right)(\xi)$, for any $\xi \in F(C-S)$, i.e., the diagram

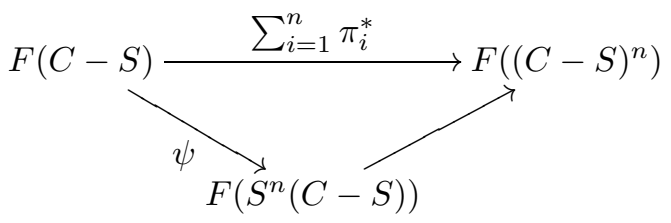

commutes. 
We claim that for any effective divisor $\delta=\sum_{j} n_{j} x_{j}$ of degree $n$ on $C-S$, we have

$$
[\delta]^{*} \circ \psi=\delta^{*} \in \operatorname{Hom}(F(C-S), F(k)) .
$$

Indeed, let $\widetilde{\delta}=\left(x_{1}, \ldots, x_{1}, x_{2}, \ldots, x_{2}, \cdots\right) \in(C-S)^{n}$, where $x_{j}$ is repeated $n_{j}$ times as a coordinate. Then $\widetilde{\delta}$ is a preimage in $(C-S)^{n}$ of $[\delta] \in S^{n}\left((C-S)^{n}\right)$. Hence

$$
\widetilde{\delta}^{*} \circ\left(\sum_{i=1}^{n} \pi_{i}^{*}\right)=[\delta]^{*} \circ \psi .
$$

On the other hand, from the definitions, it is clear that

$$
\widetilde{\delta}^{*} \circ\left(\sum_{i=1}^{n} \pi_{i}^{*}\right)=\sum_{j} n_{j} x_{j}^{*}=\delta^{*} .
$$

Now suppose $f \in k(C)$ such that $\left.f\right|_{S}=1$. Let $T=f^{-1}(1)$, and consider $f$ as a morphism $f: C-T \rightarrow \mathbb{P}^{1}-\{1\}$. There is an induced morphism

$$
\widetilde{f}: \mathbb{P}^{1}-\{1\} \rightarrow S^{n}(C-T) \hookrightarrow S^{n}(C-S),
$$

where $n=\operatorname{deg} f$. The map $\tilde{f}$ has the property that if $\delta_{t}=f^{-1}(t)$ as a divisor, then $\tilde{f}(t)=\left[\delta_{t}\right]$.

Let $\delta_{0}=(f)_{0}, \delta_{\infty}=(f)_{\infty}$ be the divisors of poles and zeroes of $f$. Then the lemma asserts that

$$
\delta_{0}^{*}=\delta_{\infty}^{*}: F(C-S) \rightarrow F(k) .
$$

To prove this, by (43), it suffices to show that

$$
\left[\delta_{0}\right]^{*}=\left[\delta_{\infty}\right]^{*}: F\left(S^{n}(C-S)\right) \rightarrow F(k) .
$$

Since $\tilde{f}(t)=\left[\delta_{t}\right]$, it follows that

$$
\left[\delta_{t}\right]^{*}=t^{*} \circ \tilde{f}^{*} \forall t \in \mathbb{P}^{1}-\{1\},
$$

and so we are reduced to proving that $0^{*}=\infty^{*}: F\left(\mathbb{P}^{1}-\{1\}\right) \rightarrow F(k)$.

If $\pi: \mathbb{P}^{1}-\{1\} \rightarrow$ Spec $k$ is the structure morphism, then we are given that

$$
\pi^{*}: F(k) \rightarrow F\left(\mathbb{P}^{1}-\{1\}\right),
$$

while clearly $0^{*} \circ \pi^{*}$ and $\infty^{*} \circ \pi^{*}$ both equal the identity on $F(k)$. Hence $0^{*}=\infty^{*}$ as desired.

Now we can easily show that our "relative" and "simplicial" Picard functors are representable by checking the list of axioms P1-P7'. Because of Lemma A.1.1 and Lemma A.1.2 representability will follows from the representability theorems for the classical Picard functor: we will sketch the arguments below.

We remark that, in the particular case when we have an extension of sheaves as above and we moreover assume that $\widetilde{F}^{\prime}$ is affine, one can then also deduce representability of $\widetilde{F}$ by descent, as in Proposition 17.4 of [38]. 


\section{A.2. Representability of the relative Picard functor}

In order to show representability of $f p q c$-sheaves one can assume that the base field $k$ is algebraically closed (see [36, Lemma I.8.9]). The fpqc-sheaf associated to the relative Picard functor $T \longmapsto \operatorname{Pic}\left(\bar{X} \times_{k} T, Y \times_{k} T\right)$ in Lemma 2.1 .1 will be denoted by $\operatorname{Pic}_{(\bar{X}, Y) / k}$. The exact sequence (5) yields the following short exact sequence of fpqc-sheaves

$$
0 \rightarrow T(\bar{X}, Y) \rightarrow \operatorname{Pic}_{(\bar{X}, Y) / k} \rightarrow \operatorname{ker}\left(\operatorname{Pic}_{\bar{X} / k} \rightarrow \operatorname{Pic}_{Y / k}\right) \rightarrow 0
$$

Since $\bar{X}$ is non-singular and complete, we see that to prove representability of $\operatorname{Pic}_{(\bar{X}, Y) / k}$, we reduce immediately to the case when $\bar{X}$ is connected, hence irreducible. If $Y=\varnothing$, then [36] yields the desired representability. If $\bar{X}$ is irreducible and $Y \neq \varnothing$ (as we may now assume), then pairs $(\mathcal{L}, \alpha)$ consisting of line bundles on $\bar{X}$, trivialized along $Y$, do not admit non-trivial automorphisms. Therefore, the functor which takes a $k$-scheme $T$ to $\operatorname{Pic}\left(\bar{X} \times_{k} T, Y \times_{k} T\right)$ is already a sheaf with respect to the Zariski topology and, by descent theory (see $[42, \S 2.1],[11, \S 8.1]$ ), even with respect to the fpqc-topology.

We now apply our Lemma A.1.1 and Lemma A.1.2 to the functors $F=\widetilde{F}=$ $\operatorname{Pic}_{(\bar{X}, Y) / k}, F^{\prime}=\widetilde{F}^{\prime}=T(\bar{X}, Y)$ and

$$
F^{\prime \prime}=\operatorname{ker}\left(\operatorname{Pic}_{\bar{X} / k} \rightarrow \operatorname{Pic}_{Y / k}\right)=\operatorname{ker}\left(\operatorname{Pic}_{\bar{X} / k} \rightarrow \oplus \operatorname{Pic}_{Y_{i} / k}\right)
$$

where $Y_{j}$ for $j=1, \ldots, r$ are the connected (possibly reducible) components of $Y$. We have that $\bar{X}$ integral and $\left(\pi_{\bar{X}}\right)_{*}\left(\mathcal{O}_{\bar{X}}\right)=\left(\pi_{Y_{j}}\right)_{*}\left(\mathcal{O}_{Y_{j}}\right)=k$. We then have the following commutative diagram with exact rows and columns

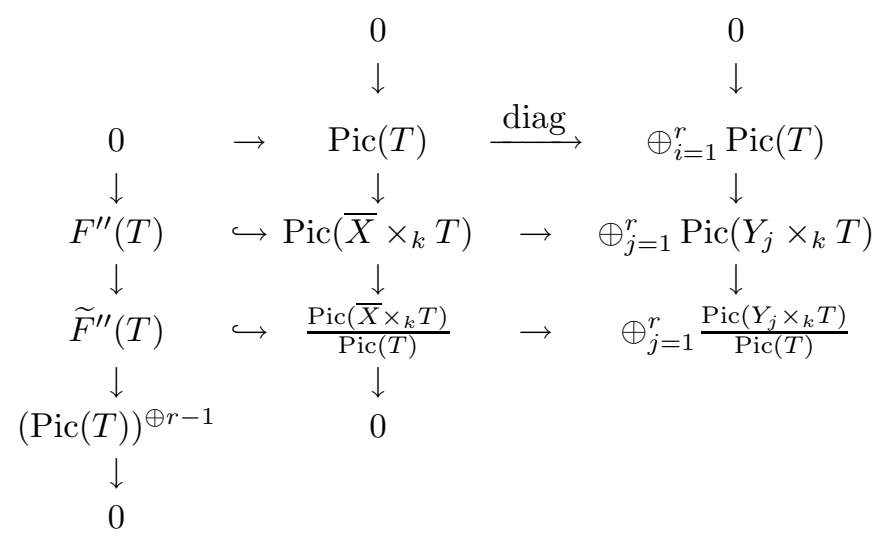

Thus we have that the functor $Q(T)$ in (41) is canonically isomorphic to $(\operatorname{Pic}(T))^{\oplus r-1}$. It is then easy to see that $Q$ satisfies the hypotheses i) - iii) stated in the Lemma A.1.1. 
Let $\coprod_{i} Y_{i} \rightarrow Y$ be the normalization of the normal crossing divisor $Y$, where $Y_{i}$ are now the irreducible components of $Y$. Consider the following exact sequence

$0 \rightarrow \operatorname{ker}\left(\operatorname{Pic}_{\bar{X} / k} \rightarrow \operatorname{Pic}_{Y / k}\right) \rightarrow \operatorname{ker}\left(\operatorname{Pic}_{\bar{X} / k} \rightarrow \oplus_{i} \operatorname{Pic}_{Y_{i} / k}\right) \stackrel{\rho}{\longrightarrow} \operatorname{ker}\left(\operatorname{Pic}_{Y / k} \rightarrow \oplus \operatorname{Pic}_{Y_{i} / k}\right)$.

In the Lemma 2.1.1 we have shown that the map $\rho$ above vanishes on the connected components of the identity yielding a description of the semi-abelian scheme $\operatorname{Pic}_{(\bar{X}, Y) / k}^{0}$.

\section{A.3. Representability of the simplicial Picard functor}

Let $X$. be a simplicial scheme. We first construct an explicit functorial isomorphism

$$
\operatorname{Pic}(X .) \stackrel{\cong}{\rightrightarrows} \mathbb{H}^{1}\left(X ., \mathcal{O}_{X}^{*}\right)
$$

as claimed in Proposition 4.1.1. We clearly can bijectively associate to (the isomorphism class of) a simplicial line bundle $\mathcal{L}$. on $X$. (i.e., to an invertible $\mathcal{O}_{X}$.module) a pair $(\mathcal{L}, \alpha)$ consisting of a line bundle $\mathcal{L}$ on $X_{0}$ and an isomorphism $\alpha:\left(d_{0}\right)^{*}(\mathcal{L}) \stackrel{\cong}{\rightrightarrows}\left(d_{1}\right)^{*}(\mathcal{L})$ on $X_{1}$, satisfying the cocycle condition (as in the Section 4.1).

Assume given:

1) an element $\xi \in \check{H}^{1}\left(\mathcal{U}, \mathcal{O}_{X_{0}}^{*}\right)$, for an open covering $\mathcal{U}=\left\{U_{i}\right\}_{i \in I}$ of $X_{0}$, corresponding to a line bundle $\mathcal{L} \in \operatorname{Pic}\left(X_{0}\right)$, together with trivializations $s_{i}: \mathcal{O}_{U_{i}} \cong$ $\left.\mathcal{L}\right|_{U_{i}}$; then $\xi=\left\{f_{i j} \in \mathcal{O}_{X_{0}}^{*}\left(U_{i} \cap U_{j}\right)\right\}$ with $\left.s_{i}\right|_{U_{i} \cap U_{j}}=\left.f_{i j} s_{j}\right|_{U_{i} \cap U_{j}}$

2) an isomorphism $\alpha:\left(d_{0}\right)^{*}(\mathcal{L}) \stackrel{\cong}{\cong}\left(d_{1}\right)^{*}(\mathcal{L})$ satisfying the cocycle condition. Let

$$
V_{i j} \stackrel{\text { def }}{=} d_{1}^{-1}\left(U_{i}\right) \cap d_{0}^{-1}\left(U_{j}\right)
$$

Then $\left\{V_{i j}\right\}_{(i, j) \in I \times I}$ is an open covering of $X_{1}$. Moreover, on $V_{i j}$ we have trivializations $d_{1}^{*}\left(s_{i}\right)$ of $\left(d_{1}\right)^{*}(\mathcal{L})$, and $d_{0}^{*}\left(s_{j}\right)$ of $\left(d_{0}\right)^{*}(\mathcal{L})$, respectively. Therefore, $\alpha$ is uniquely determined by $\alpha_{i j} \in \mathcal{O}_{X_{1}}^{*}\left(V_{i j}\right)$, satisfying

$$
d_{1}^{*}\left(s_{i}\right)=\alpha_{i j} d_{0}^{*}\left(s_{j}\right)
$$

on $V_{i j}$. The $\alpha_{i j}$ have to satisfy a compatibility condition: on $V_{i j} \cap V_{k l}$, have $d_{1}^{*}\left(s_{i}\right)=$ $d_{1}^{*}\left(f_{i k}\right) d_{1}^{*}\left(s_{k}\right)$, and $d_{0}^{*}\left(s_{j}\right)=d_{0}^{*}\left(f_{j l}\right) d_{0}^{*}\left(s_{l}\right)$, thus

$$
d_{1}^{*}\left(s_{i}\right)=d_{1}^{*}\left(f_{i k}\right) d_{1}^{*}\left(s_{k}\right)=d_{1}^{*}\left(f_{i k}\right) \alpha_{k l} d_{0}^{*}\left(s_{l}\right),
$$

but $d_{1}^{*}\left(s_{i}\right)=\alpha_{i j} d_{0}^{*}\left(s_{j}\right)=\alpha_{i j} d_{0}^{*}\left(f_{j l}\right) d_{0}^{*}\left(s_{l}\right)$ as well, therefore $d_{0}^{*}\left(s_{l}\right)$ cancels and we obtain

$$
d_{1}^{*}\left(f_{i k}\right) \alpha_{k l}=\alpha_{i j} d_{0}^{*}\left(f_{j l}\right)
$$

on $V_{i j} \cap V_{k l}$. 
Let $\mathcal{K}_{i}$ be the canonical (Godement) flasque sheaf of discontinuous sections of $\mathcal{O}_{X_{i}}^{*}$ and let $\mathcal{Q}_{i} \stackrel{\text { def }}{=} \frac{\mathcal{K}_{i}}{\mathcal{O}_{X_{i}}^{*}}$ denote the quotient sheaf. We have an exact sequence

$$
0 \rightarrow \Gamma\left(X_{i}, \mathcal{O}_{X_{i}}^{*}\right) \rightarrow \Gamma\left(X_{i}, \mathcal{K}_{i}\right) \rightarrow \Gamma\left(X_{i}, \mathcal{Q}_{i}\right) \rightarrow H^{1}\left(X_{i}, \mathcal{O}_{X_{i}}^{*}\right) \rightarrow 0
$$

Choose a function $\varphi: X_{0} \rightarrow I$ such that $x \in U_{\varphi(x)}$ for any $x \in X$. This determines well defined sections $t_{i} \in \mathcal{K}_{0}\left(U_{i}\right), t_{i}(x)=f_{i \varphi(x)} \in \mathcal{O}_{X_{0}, x}^{*}$. For any $x \in U_{i} \cap U_{j}$, we have $f_{i j}=f_{i \varphi(x)} f_{j \varphi(x)}^{-1} \in \mathcal{O}_{X_{0}, x}^{*}$, therefore: $t_{i}=f_{i j} t_{j}$ on $U_{i} \cap U_{j}$ and the images of $t_{i}$ in $\mathcal{Q}_{0}\left(U_{i}\right)$ patch together to give a global section $t \in \Gamma\left(X_{0}, \mathcal{Q}_{0}\right)$. By construction,

$$
[t] \in \frac{\Gamma\left(X_{0}, \mathcal{Q}_{0}\right)}{\operatorname{im} \Gamma\left(X_{0}, \mathcal{K}_{0}\right)}=H^{1}\left(X_{0}, \mathcal{O}_{X_{0}}^{*}\right)
$$

is the class of the given line bundle $\mathcal{L}$ on $X_{0}$.

Next, we have a natural element $\beta \in \Gamma\left(X_{1}, \mathcal{K}_{1}\right)$, given by

$$
\beta(x)=\alpha_{\varphi\left(d_{1}(x)\right) \varphi\left(d_{0}(x)\right)} \in \mathcal{O}_{X_{0}, x}^{*}
$$

note that $x \in V_{\varphi\left(d_{1}(x)\right) \varphi\left(d_{0}(x)\right)}$ by the definition of $\varphi$. We claim that (writing the group operation in $\Gamma\left(X_{1}, \mathcal{Q}_{1}\right)$ multiplicatively):

$$
[\beta]=\frac{d_{0}^{*}(t)}{d_{1}^{*}(t)} \in \frac{\Gamma\left(X_{1}, \mathcal{K}_{1}\right)}{\Gamma\left(X_{1}, \mathcal{O}_{X_{1}}^{*}\right)} \subset \Gamma\left(X_{1}, \mathcal{Q}_{1}\right) .
$$

In fact, on $V_{i j}$ we have that $d_{1}^{*}(t)$ is the image of $d_{1}^{*}\left(t_{i}\right)$ where $d_{1}^{*}\left(t_{i}\right)(x)=$ $d_{1}^{*}\left(f_{i \varphi\left(d_{1}(x)\right)}\right) \in \mathcal{O}_{X_{1}, x}^{*}$; similarly, $d_{0}^{*}(t)$ is the image of $d_{0}^{*}\left(t_{j}\right)$ where $d_{0}^{*}\left(t_{j}\right)(x)=$ $d_{0}^{*}\left(f_{j \varphi\left(d_{0}(x)\right)}\right) \in \mathcal{O}_{X_{1}, x}^{*}$. From the definition of $\mathcal{Q}_{1}$ as a quotient sheaf, the claimed formula (45) will be proved if: for any $i, j \in I$ and $x \in V_{i j}, d_{1}^{*}\left(t_{i}\right)\left(d_{0}^{*}\left(t_{j}\right)\right)^{-1} \beta(x)$ defines a section in $\mathcal{O}_{X_{1}}^{*}\left(V_{i j}\right)$. From the identity (44) we have

$$
d_{1}^{*}\left(t_{i}\right)\left(d_{0}^{*}\left(t_{j}\right)\right)^{-1} \beta(x)=d_{1}^{*}\left(f_{i \varphi\left(d_{1}(x)\right)}\right)\left(d_{0}^{*}\left(f_{j \varphi\left(d_{0}(x)\right)}\right)\right)^{-1} \alpha_{\varphi\left(d_{1}(x)\right) \varphi\left(d_{0}(x)\right)}=\alpha_{i j} \in \mathcal{O}_{X_{1}, x}^{*}
$$

Thus, given a simplicial line bundle, therefore data as in 1) and 2) above, together with a choice of $\varphi: X \rightarrow I$ we get an element of $\mathbb{H}^{1}\left(X ., \mathcal{O}_{X}^{*}\right)$ computed by means of the canonical Godement resolution of the simplicial sheaf $\mathcal{O}_{X}^{*}$.

It is now easy to verify that this construction is independent of the additional choices made (the local trivializations $s_{i}$ and the map $\varphi$ ), and defines a homomorphism

$$
\operatorname{Pic}(X .) \rightarrow \mathbb{H}^{1}\left(X ., \mathcal{O}_{X}^{*}\right) \text {. }
$$

Conversely, we see that $\mathbb{H}^{1}\left(X ., \mathcal{O}_{X}^{*}\right)$ is identified with the $H^{1}$ of the following complex

$$
\Gamma\left(X_{0}, \mathcal{K}_{0}\right) \rightarrow \Gamma\left(X_{1}, \mathcal{K}_{1}\right) \oplus \Gamma\left(X_{0}, \mathcal{Q}_{0}\right) \rightarrow \Gamma\left(X_{2}, \mathcal{K}_{2}\right) \oplus \Gamma\left(X_{1}, \mathcal{Q}_{1}\right) .
$$

Given a cycle $(\beta, t) \in \Gamma\left(X_{1}, \mathcal{K}_{1}\right) \oplus \Gamma\left(X_{0}, \mathcal{Q}_{0}\right)$, we can choose an open cover $\left\{U_{i}\right\}$ of $X_{0}$ and pre-images $t_{i} \in \mathcal{K}_{0}\left(U_{i}\right)$ of $t$, and we will then obtain $f_{i j} \in \mathcal{O}_{X_{0}}^{*}\left(U_{i} \cap U_{j}\right)$ satisfying $t_{i}=f_{i j} t_{j}$ in $\mathcal{K}_{0}\left(U_{i} \cap U_{j}\right)$. Now one immediately verifies that the $f_{i j}$ define an invertible sheaf $\mathcal{L}$ on $X_{0}$, and (reversing the earlier arguments) $\beta$ determines an 
isomorphism $\alpha: d_{0}^{*} \mathcal{L} \cong d_{1}^{*} \mathcal{L}$. Since $\beta$ maps to 0 in $\Gamma\left(X_{2}, \mathcal{K}_{2}\right)$, we deduce that $\alpha$ satisfies the cocycle condition.

We now come to the proof of the representability of the simplicial Picard functor. Let $X$. be a smooth and proper simplicial $k$-scheme. To prove representability of the simplicial Picard functor, we again reduce to the case when $k$ is algebraically closed, using [36, Lemma I.8.9]. Then we may further reduce to the case when $\left(\pi_{X}\right)_{*}\left(\mathcal{O}_{X_{.}}\right)=k$. Then we have

$$
\operatorname{Pic}_{X . / k}(T)=\frac{\operatorname{Pic}\left(X \cdot \times_{k} T\right)}{\operatorname{Pic} T}
$$

since we can choose a base point in $X$. We let $\mathbb{Z}^{X_{a}}$ denote the free abelian group on the connected components of $X_{a}, a=0,1, \ldots$, and let $\pi_{a}: X_{a} \rightarrow k$ be the structural morphism.

We then set ( $c f$. Chapter 4)

$$
\begin{aligned}
& K \stackrel{\text { def }}{=} \operatorname{ker}\left(\mathbb{Z}^{X_{0}} \rightarrow \mathbb{Z}^{X_{1}}\right) \\
& C \stackrel{\text { def }}{=} \operatorname{coker}\left(\mathbb{Z}^{X_{0}} \rightarrow \mathbb{Z}^{X_{1}}\right) \\
& F^{\prime} \stackrel{\text { def }}{=} T(\bar{X} .) \stackrel{\text { def }}{=} \frac{\operatorname{ker}\left(\left(\pi_{1}\right)_{*} \mathbb{G}_{m, X_{1}} \rightarrow\left(\pi_{2}\right)_{*} \mathbb{G}_{m, X_{2}}\right)}{\operatorname{im}\left(\left(\pi_{0}\right)_{*} \mathbb{G}_{m, X_{0}} \rightarrow\left(\pi_{1}\right)_{*} \mathbb{G}_{m, X_{1}}\right)} \\
& F(T) \stackrel{\text { def }}{=} \operatorname{Pic}\left(X . \times_{k} T\right) \\
& \widetilde{F}=\mathbf{P i c}_{X} \cdot / k \\
& F^{\prime \prime}(T) \stackrel{\text { def }}{=} \operatorname{ker}\left(\operatorname{Pic}\left(X_{0} \times_{k} T\right) \rightarrow \operatorname{Pic}\left(X_{1} \times_{k} T\right)\right)
\end{aligned}
$$

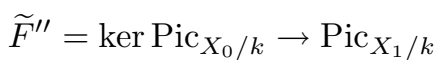

$$
\begin{aligned}
& G \stackrel{\text { def }}{=} \frac{\operatorname{ker}\left(\left(\pi_{2}\right)_{*} \mathbb{G}_{m, X_{2}} \rightarrow\left(\pi_{3}\right)_{*} \mathbb{G}_{m, X_{3}}\right)}{\operatorname{im}\left(\left(\pi_{1}\right)_{*} \mathbb{G}_{m, X_{1}} \rightarrow\left(\pi_{2}\right)_{*} \mathbb{G}_{m, X_{2}}\right)} \\
& \widetilde{G}=\text { associated } f p q c \text { sheaf. }
\end{aligned}
$$

We then have an exact sequence of pre-sheaves

$$
0 \rightarrow F^{\prime} \rightarrow F \rightarrow F^{\prime \prime} \rightarrow G
$$

and, for each $T \in \mathcal{S} c h_{k}$, a commutative diagram of complexes

$$
\begin{aligned}
& 0 \rightarrow F^{\prime}(T) \rightarrow F(T) \rightarrow F^{\prime \prime}(T) \rightarrow G(T)
\end{aligned}
$$

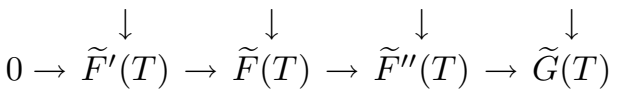

with exact top row. By (46), we have that $F(T) \rightarrow \widetilde{F}(T)$ is surjective with kernel $\operatorname{Pic}(T)$. Moreover we have the following commutative diagram with exact rows and 
columns:

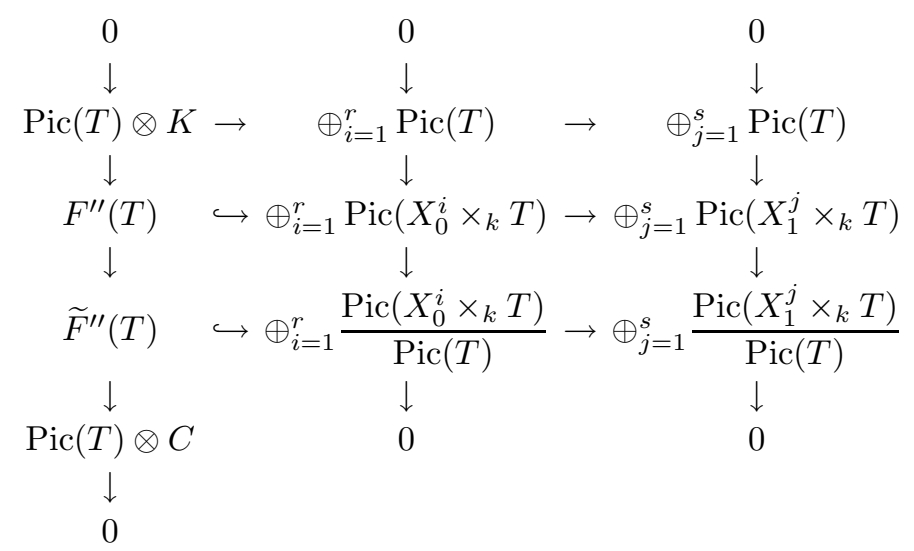

Now we can see that $F^{\prime}(T) \cong \widetilde{F}^{\prime}(T)$ in fact: $F^{\prime}$ is of $\mathbb{G}_{m}$-type whence the map $F^{\prime}(T) \rightarrow \widetilde{F}^{\prime}(T)$ is surjective with finite kernel but the finite kernel is actually zero being isomorphic to the kernel of the injective map $\operatorname{Pic}(T) \hookrightarrow \operatorname{Pic}(T) \otimes K$. Now we let $G^{\prime}$ denote the image of $F$ in $F^{\prime \prime}$. The associated $f p q c$-sheaf $\widetilde{G}^{\prime}$ is representable, in fact: $\widetilde{G}^{\prime}$ is the kernel of the homomorphism of group schemes $\widetilde{F}^{\prime \prime} \rightarrow \widetilde{G}$. We then have the following commutative diagram with exact rows:

$$
\begin{aligned}
0 & \rightarrow F^{\prime}(T) \rightarrow F(T) \rightarrow G^{\prime}(T) \rightarrow 0 \\
& \downarrow \\
& \downarrow \\
0 & \rightarrow \widetilde{F}^{\prime}(T) \rightarrow \widetilde{F}(T) \rightarrow \widetilde{G}^{\prime}(T) \rightarrow Q(T) \rightarrow 0
\end{aligned}
$$

where, by definition, $Q(T)$ is the cokernel of $\widetilde{F}(T) \rightarrow \widetilde{G}^{\prime}(T)$ and we can apply our Lemma A.1.1 and Lemma A.1.2. As $G$ is of $\mathbb{G}_{m}$-type then the map $G(T) \rightarrow \widetilde{G}(T)$ is surjective with finite kernel $G_{0}(T)$. Moreover we have that $\operatorname{Pic}(T)$ and $Q(T)$ are respectively the kernel and the cokernel of $G^{\prime}(T) \rightarrow \widetilde{G}^{\prime}(T)$. Considering $\widetilde{G}^{\prime}$ as the kernel of the homomorphism of group schemes $\widetilde{F}^{\prime \prime} \rightarrow \widetilde{G}$ we can see that there is a functorial exact sequence

$$
0 \rightarrow \text { finite group } \rightarrow Q(T) \rightarrow \operatorname{Pic}(T) \otimes C
$$

where the finite group is a subgroup of $G_{0}(T)$ whence it is zero whenever $H^{0}\left(T, \mathcal{O}_{T}^{*}\right)$ is divisible $e . g$., if $T$ is an artinian algebra or a strictly Hensel local ring.

Therefore we can easily check the vanishing conditions of Lemma A.1.1: i) ii) follow from (47), and iii) follows by a diagram chase using (47) since Pic and $H^{0}\left(T, \mathcal{O}_{T}^{*}\right)$ commute with the relevant direct limits. 



\section{BIBLIOGRAPHY}

[1] L. BARBIERI-VIALE - On algebraic 1-motives related to Hodge cycles, preprint math.AG/0103179, 2001.

[2] L. Barbieri-Viale, C. Pedrini and C. Weibel - Roitman's theorem for singular complex projective surfaces, Duke Math. J. 84, No. 1, (1996) 155-189.

[3] L. Barbieri-Viale, A. Rosenschon and M. Saito - Deligne's conjecture on 1-motives, preprint math.AG/0102150, 2001.

[4] L. Barbieri-Viale and V. SRinivas - On the Néron-Severi group of a singular variety, J. Reine Ang. Math. (Crelles Journal) 435 (1993) 65-82.

[5] L. Barbieri-Viale and V. SRinivas - The Néron-Severi group and the mixed Hodge structure on $H^{2}$, J. Reine Ang. Math. (Crelles Journal) 450 (1994) 37-42.

[6] L. Barbieri-Viale and V. Srinivas - Albanese and Picard 1-motives, $C$. $R$. Acad. Sci. Paris, t. 326, Série I (1998) 1397-1401.

[7] J. BIswas - Topics in Algebraic Cycles, PhD Thesis, TIFR and University of Mumbai, 1997.

[8] J. Biswas and V. SRINIVAS - Roitman's theorem for singular projective varieties, Compositio Math. 119 (1999) 213-237.

[9] J. Biswas and V. SRINIVAS - A Lefschetz $(1,1)$-Theorem for normal projective complex varieties, Duke Math. J. 101 (2000) 427-458.

[10] J-F. Boutot - Schéma de Picard local, Springer LNM 632, 1978.

[11] S. Bosch, W. Lutkebohmert and M. Raynaud - Néron Models, Springer Ergebnisse der Math. 21 Heidelberg, 1990.

[12] J.A. CARLSON - The one-motif of an algebraic surface, Compositio Math. 56 (1985) 271-314. 
[13] C. Chevalley - Sur la théorie de la variété de Picard, American J. Math. 82 (1960) 435-490.

[14] P. Deligne - Equations différentielles à points singuliers réguliers, Springer LNM 163 Heidelberg, 1970.

[15] P. Deligne - Théorie de Hodge II, III Publ. Math. IHES 40 (1972) 5-57 and Publ. Math. IHES 44 (1974) 5-78.

[16] P. Deligne - A quoi servent les motifs ?, in "Motives" Proc. Symp. Pure Math AMS 55, Part 1, (1994) 143-161.

[17] P. Deligne - Letter to H. Esnault, November 4, 1994.

[18] P. Deligne and L. Illusie - Rélèvements modulo $p^{2}$ et décomposition du complexe de de Rham, Invent. Math. 89 (1987) 247-270.

[19] H. Esnault, V. SRinivas and E. Viehweg - The universal regular quotient of the Chow group of points on projective varieties, Inv. Math. 135 (1999) 595-664.

[20] H. Esnault and E. Viehweg - Lectures on vanishing theorems, Birkäuser DMV Seminar 20 Basel, 1992.

[21] W. Fulton - Intersection theory, Springer Ergebnisse der Math. 2 Heidelberg, 1984.

[22] H. Gillet - On the $K$-theory of surfaces with multiple curves and a conjecture of Bloch, Duke Math. J. 51, No. 1, (1984) 195-233.

[23] A. Grothendieck - Technique de descente et théorèmes d'existence en géométrie algébrique I-VI, "Fondements de la Géométrie Algébrique" Extraits du Séminaire Bourbaki 1957/62.

[24] A. Grothendieck et al. - SGA2 - Cohomologie locale des faisceaux cohérents et théorèmes de Lefschetz locaux et globaux (1962), North-Holland, Amsterdam, 1968.

[25] A. Grothendieck et al. - SGA4 - Théorie des topos et cohomologie étale des schémas (1963-64), Springer LNM 269270 305, Heidelberg, 1972-73.

[26] A. Grothendieck et al. - SGA6 - Théorie des intersections et théorème de Riemann-Roch (1966-67), Springer LNM 589, 1977.

[27] A. Grothendieck et al. - SGA7 - Groupes de monodromie en géométrie algébrique (1967-68), Springer LNM 288 340, 1972-73.

[28] R. HARTShorne - Ample subvarieties of algebraic varieties, Springer LNM 156, 1970.

[29] R. Hartshorne - On the De Rham cohomology of algebraic varieties, Publ. Math. IHES 45 (1976) 5-99. 
[30] M. LEVINE and C. WeIBEL - Zero-cycles and complete intersections on singular varieties, J. Reine Ang. Math. (Crelles Journal) 359 (1985) 106-120.

[31] S. Lichtenbaum - Suslin homology and Deligne 1-motives, in "Algebraic $K$ theory and algebraic topology" NATO ASI Series, 1993, 189-197.

[32] B. Mazur and W. Messing - Universal extensions and one dimensional crystalline cohomology, Springer LNM 370, 1974.

[33] J. S. Milne - Étale cohomology, Princeton University Press, Princeton, New Jersey, 1980.

[34] D. Mumford - Biextensions of formal groups, International Colloquium on Algebraic Geometry (Bombay, 1968) Oxford University Press, 1969, 307-322.

[35] D. Mumford - The topology of normal singularities on an algebraic surface and a criterion for simplicity, Publ. Math. IHES 9 (1961) 5-22.

[36] J. MURRE - On contravariant functors from the category of preschemes over a field into the category of abelian groups (with application to the Picard functor), Publ. Math. IHES 23 (1964) 581-619.

[37] F. OORT - A construction of generalized Jacobian varieties by group extensions, Math. Annalen 147 (1962) 277-286.

[38] F. OorT - Commutative group schemes, Springer LNM 15, 1966.

[39] N. RAmachandran - Albanese and Picard 1-motives, Ph D Thesis (preliminary version, 1995).

[40] N. RAmachandran - Albanese and Picard one-motives of schemes, preprint, math.AG/9804042, 1998.

[41] N. RAmachandran - A conjecture of Deligne on one-motives, preprint, math.AG/9806117, 1998.

[42] M. Raynaud - Spécialisation du foncteur de Picard, Publ. Math. IHES 38 (1970) 27-76.

[43] M. RAYNAUd - 1-motifs et monodromie géométrique, Exposé VII, Astérisque 223 (1994) 295-319.

[44] M. Saito - Mixed Hodge modules, Publ. RIMS Kyoto Univ. 26 (1990) 221-333.

[45] M. SAITo - Introduction to mixed Hodge modules, Astérisque, 179-180 (1989) 145-162.

[46] S. SAITo - Torsion zero-cycles and étale homology of singular schemes, Duke Math. J. 64, No. 1, (1991) 71-83. 
[47] J.-P. SERRE - Morphismes universels et variétés d'Albanese, dans "Variétés de Picard" ENS Séminaire C. Chevalley 3e année: 1958/59.

[48] J.-P. SERRE - Morphismes universels et différentielles de troisième espèce, dans "Variétés de Picard" ENS Séminaire C. Chevalley 3e année: 1958/59.

[49] J.-P. Serre - Groupes algébriques et corps de classes, Hermann, Paris, 1959.

[50] E. H. Spanier - Algebraic Topology, McGraw-Hill, New York, 1966.

[51] J.H.M. SteEnBRink - Cup products and mixed Hodge structures, preprint, math.AG/9902134, 1999.

[52] A. SusLin and V. Voevodsky - Singular homology of abstract algebraic varieties, Inv. Math. 123 (1996) 61-94.

[53] Bin Zhang - Sur les jacobiennes des courbes à singularités ordinaires, Manuscripta Math. 92 (1997) 1-12. 